\title{
The Quest for Functional Oxide Cathodes for Magnesium Batteries: A Critical Perspective
}

Ian D. Johnson ${ }^{1,2}$, Brian J. Ingram ${ }^{1,2}$, Jordi Cabana ${ }^{2,3, *}$

${ }^{1}$ Chemical Sciences and Engineering Division, Argonne National Laboratory, Lemont, Illinois 60439, United States

2 Joint Center for Energy Storage Research, Argonne National Laboratory, Lemont, IL 60439, USA.

${ }^{3}$ Department of Chemistry, University of Illinois at Chicago, Chicago, IL 60607, USA.

Supporting Information 
Table 1. List of acronyms

\begin{tabular}{|c|c|}
\hline Acronym & Meaning \\
\hline ABF-STEM & Annular bright-field scanning transmission electron microscopy \\
\hline APC & All phenyl complex \\
\hline ATR-FTIR & Attenuated total reflection Fourier transform infrared spectroscopy \\
\hline AZ31 & Mg alloy with 3 wt\% Al and 1 wt\% Zn \\
\hline AZ61 & Mg alloy with 6 wt\% Al and 1 wt\% Zn \\
\hline$[\mathrm{bim}] \mathrm{Br}$ & 1, 3-dibutyl imidazolium bromide \\
\hline BMP & 1-butyl-1-methylpyrrolidinium \\
\hline DMC & Dimethylcarbonate \\
\hline DME & Dimethoxyethane \\
\hline $\mathrm{DMSO}_{2}$ & Dimethyl sulfone \\
\hline EA & Ethyl acetate \\
\hline EC & Ethylene carbonate \\
\hline EDS/EDX & Energy-dispersive X-ray spectroscopy \\
\hline EELS & Electron energy loss spectroscopy \\
\hline EMC & Ethyl methyl carbonate \\
\hline EMIC & 1-ethyl-3-methylimidazolicum \\
\hline EPR & Electron paramagnetic resonance \\
\hline EQCM & Electrochemical quartz crystal microbalance \\
\hline EXAFS & Extended X-ray absorption fine structure \\
\hline FTIR & Fourier transform infrared spectroscopy \\
\hline G4 & Tetraglyme \\
\hline HAADF-STEM & High-angle annular dark-field imaging scanning transmission electron microscopy \\
\hline HMDS & Hexamethyldisilazane \\
\hline HRSEM & High-Resolution Scanning Electron Microscopy \\
\hline HR-TEM & High resolution transmission electron microscopy \\
\hline ICP & Inductively coupled plasma \\
\hline ICP-AES & Inductively coupled plasma atomic emission spectroscopy \\
\hline ICP-MS & Inductively coupled plasma mass spectrometry \\
\hline
\end{tabular}




\begin{tabular}{|c|c|}
\hline Acronym & Meaning \\
\hline ICP-OES & inductively coupled plasma optical emission spectrometry \\
\hline NMC & Nickel manganese cobalt \\
\hline NMR & Nuclear magnetic resonance \\
\hline $\mathrm{P} 13 / \mathrm{PYR}_{13}$ & N-Propyl-N-methylpyrrolidinium \\
\hline PA & Phenylamine \\
\hline $\mathrm{PC}$ & Propylene carbonate \\
\hline PDF & Pair distribution function \\
\hline PEO & Polyethlyene oxide \\
\hline $\mathrm{PY}_{14}$ & 1-Butyl-1-methylpyrrolidinium \\
\hline SAXS & Small-angle X-ray scattering \\
\hline SEM & Scanning electron microscopy \\
\hline SS-NMR & Solid-state nuclear magnetic resonance \\
\hline STEM & Scanning transmission electron microscopy \\
\hline STXM & Scanning transmission X-ray microscopy \\
\hline TEM & Transmission electron microscopy \\
\hline TFSI/TFSA & bis(trifluoromethanesulfonyl)imide \\
\hline TGA & Thermogravimetric analysis \\
\hline THF & Tetrahydrofuran \\
\hline TPFA & tetrakis(perfluoro-tert-butoxy)aluminate \\
\hline UV-vis & Ultraviolet-visible \\
\hline WAXS & Wide-angle X-ray scattering \\
\hline XANES & X-ray absorption near edge structure \\
\hline XAS & X-ray absorption spectroscopy \\
\hline XPS & X-ray photoelectron spectroscopy \\
\hline $\mathrm{XRD}$ & X-ray diffraction \\
\hline
\end{tabular}


Table 2. List of literature reports of $\mathrm{Mg}$ intercalation in metal oxide cathodes

\begin{tabular}{|c|c|c|c|c|c|c|}
\hline Material & $\begin{array}{c}\text { Cycling } \\
\text { conditions: } \\
\text { Temperature, } \\
\text { anode, and } \\
\text { electrolyte } \\
\end{array}$ & $\begin{array}{l}\text { Magnesiated } \\
\text { Product phases }\end{array}$ & Redox changes & $\begin{array}{c}\text { Reported } \\
\text { Electrochemical } \\
\text { Capacity }\end{array}$ & $\begin{array}{l}\text { Experimentally } \\
\text { Verified } \\
\text { Magnesiated } \\
\text { Composition }\end{array}$ & Reference \\
\hline $\mathrm{MgCr}_{2} \mathrm{O}_{4}$ & $\begin{array}{l}50^{\circ} \mathrm{C} \text {, Li foil, } 1.0 \mathrm{M} \\
\mathrm{LiPF}_{6} \text { in a } 3: 7 \text { (wt } \\
\% / w t \% \text { ) EC/EMC } \\
\text { mixture }\end{array}$ & $\begin{array}{l}\text { Spinel } \mathrm{Mg}_{1-x} \mathrm{Cr}_{2} \mathrm{O}_{4} \text {, } \\
\text { decreased unit } \\
\text { cell volume with } \\
\text { Mg extraction. } \\
\text { (XRD) }\end{array}$ & $\begin{array}{l}\text { Conversion of } \mathrm{Cr}^{3+} \\
\text { to } \mathrm{Cr}^{4+}\left({ }^{25} \mathrm{Mg}-\right. \\
\mathrm{NMR})\end{array}$ & $\begin{array}{l}280 \mathrm{~mA} \mathrm{~h} \mathrm{~g}^{-1} \\
\text { (charge) }\end{array}$ & $\begin{array}{l}\mathrm{Mg}_{0.29} \mathrm{Cr}_{2} \mathrm{O}_{4}\left({ }^{25} \mathrm{Mg}-\right. \\
\mathrm{NMR})\end{array}$ & S1 \\
\hline $\mathrm{MgCr}_{2} \mathrm{O}_{4}$ & $\begin{array}{l}110^{\circ} \mathrm{C} \text {, capacitive } \\
\text { carbon, } 0.5 \mathrm{M} \\
\mathrm{MgTFSI}_{2} \text { in } \\
\mathrm{PY}_{14} \mathrm{TFSI}\end{array}$ & $\begin{array}{l}\text { Spinel } \mathrm{Mg}_{1-x} \mathrm{Cr}_{2} \mathrm{O}_{4}, \\
\text { decreased unit } \\
\text { cell volume with } \\
\text { Mg extraction. } \\
\text { (XRD) }\end{array}$ & $\begin{array}{l}\text { Reversible } \\
\text { conversion of } \mathrm{Cr}^{3+} \\
\text { to } \mathrm{Cr}^{4+} \text { (XANES) }\end{array}$ & $\begin{array}{l}120 \mathrm{~mA} \mathrm{~h} \mathrm{~g} \\
\text { (charge), } 270 \mathrm{~mA} \\
\mathrm{~h} \mathrm{~g}^{-1} \text { (discharge) }\end{array}$ & $\begin{array}{l}\mathrm{Mg}_{0.75} \mathrm{Cr}_{2} \mathrm{O}_{4} \\
\text { (charge) (EDX) }\end{array}$ & $\mathrm{S} 2$ \\
\hline $\mathrm{MgCrVO}_{4}$ & $\begin{array}{l}110^{\circ} \mathrm{C}, \mathrm{Mg} \text { foil, } \\
0.1 \mathrm{M} \mathrm{Mg}(\mathrm{TPFA})_{2} \\
\text { in triglyme }\end{array}$ & $\begin{array}{l}\text { Spinel lattices } \\
\text { contracted with } \\
\text { charge, expanded } \\
\text { with discharge } \\
\text { (XRD) }\end{array}$ & $\begin{array}{l}\text { Oxidation of } \mathrm{V}^{3+} \text { to } \\
\text { a mixture of } \mathrm{V}^{4+} \\
\text { and } \mathrm{V}^{5+} \text { on charge, } \\
\text { which was } \\
\text { reversible (at least } \\
\text { on the surface). Cr } \\
\text { was inactive. } \\
\text { (XAS) }\end{array}$ & $\begin{array}{l}100 \mathrm{~mA} \mathrm{~h} \mathrm{~g} \mathrm{~g}^{-1} \text { (first } \\
\text { charge), } 80 \mathrm{~mA} \mathrm{~h} \\
\mathrm{~g}^{-1} \text { (first } \\
\text { discharge). } 40 \mathrm{~mA} \\
\mathrm{~h} \mathrm{~g}^{-1} \text { (second cycle } \\
\text { charge and } \\
\text { discharge } \\
\text { capacity) }\end{array}$ & $\begin{array}{l}\mathrm{Mg}_{0.3} \mathrm{CrVO}_{4} \\
\text { (charge) } \\
\mathrm{Mg}_{0.45} \mathrm{CrVO}_{4} \\
\text { (discharge) }\left({ }^{25} \mathrm{Mg}-\right. \\
\mathrm{NMR} \text { ) }\end{array}$ & S3 \\
\hline
\end{tabular}




\begin{tabular}{|c|c|c|c|c|c|c|}
\hline Material & $\begin{array}{c}\text { Cycling } \\
\text { conditions: } \\
\text { Temperature, } \\
\text { anode, and } \\
\text { electrolyte }\end{array}$ & $\begin{array}{l}\text { Magnesiated } \\
\text { Product phases }\end{array}$ & Redox changes & $\begin{array}{c}\text { Reported } \\
\text { Electrochemical } \\
\text { Capacity }\end{array}$ & $\begin{array}{l}\text { Experimentally } \\
\text { Verified } \\
\text { Magnesiated } \\
\text { Composition }\end{array}$ & Reference \\
\hline $\mathrm{MgCrMnO}_{4}$ & $\begin{array}{l}95^{\circ} \mathrm{C}, \mathrm{Mg} \text { foil, } 0.1 \\
\mathrm{M} \mathrm{Mg}(\mathrm{TPFA})_{2} \text { in } \\
\text { triglyme }\end{array}$ & $\begin{array}{l}\text { Spinel lattices } \\
\text { contracted with } \\
\text { charge, expanded } \\
\text { with discharge. } \\
\text { Formation of two } \\
\text { different cubic } \\
\text { spinel phases } \\
\text { (with different } \\
\text { lattice constants) } \\
\text { on discharge. } \\
\text { Rietveld } \\
\text { refinement } \\
\text { revealed Mg } \\
\text { occupation on the } \\
\text { 16d site of the } \\
\text { discharged spinel. } \\
\text { (XRD) Mg } \\
\text { vacancies evident } \\
\text { in charged state, } \\
\text { rock-salt surface } \\
\text { layers } \sim \mathrm{nm} \text { thick } \\
\text { evident in } \\
\text { discharged state } \\
\text { (STEM-EDX) }\end{array}$ & $\begin{array}{l}\text { Oxidation of } \mathrm{Mn}^{3+} \\
\text { to } \mathrm{Mn}^{3.3+} \text { on } \\
\text { charge, reduction } \\
\text { to } \mathrm{Mn}^{2.7+} \text { on } \\
\text { discharge (XAS) }\end{array}$ & $\begin{array}{l}140 \mathrm{~mA} \mathrm{~h} \mathrm{~g}^{-1} \text { (first } \\
\text { charge), } 110 \mathrm{~mA} \mathrm{~h} \\
\mathrm{~g}^{-1} \text { (first } \\
\text { discharge) }\end{array}$ & $\begin{array}{l}\mathrm{Mg}_{0.7} \mathrm{CrMnO}_{4} \\
\text { (charge), } \\
\mathrm{Mg}_{1.2} \mathrm{CrMnO}_{4} \\
\text { (discharge). (EDS) }\end{array}$ & S4 \\
\hline
\end{tabular}




\begin{tabular}{|c|c|c|c|c|c|c|}
\hline Material & $\begin{array}{c}\text { Cycling } \\
\text { conditions: } \\
\text { Temperature, } \\
\text { anode, and } \\
\text { electrolyte } \\
\end{array}$ & $\begin{array}{l}\text { Magnesiated } \\
\text { Product phases }\end{array}$ & Redox changes & $\begin{array}{c}\text { Reported } \\
\text { Electrochemical } \\
\text { Capacity }\end{array}$ & $\begin{array}{c}\text { Experimentally } \\
\text { Verified } \\
\text { Magnesiated } \\
\text { Composition }\end{array}$ & Reference \\
\hline $\mathrm{MgCo}_{2} \mathrm{O}_{4}$ & $\begin{array}{l}\text { Room } \\
\text { temperature, } \mathrm{Mg} \\
\text { foil, } 1 \mathrm{M} \\
\mathrm{Mg}\left(\mathrm{ClO}_{4}\right)_{2} \text { in } \\
\text { acetonitrile. }\end{array}$ & $\begin{array}{l}\text { No obvious } \\
\text { changes (XRD) }\end{array}$ & Not provided & Not provided & $\begin{array}{l}\text { Not reported, but } \\
\text { Mg extraction } \\
\text { verified by } \\
\text { observed } \mathrm{Mg} \\
\text { plating in a Li-ion } \\
\text { cell }\end{array}$ & S5 \\
\hline $\mathrm{MgCo}_{2} \mathrm{O}_{4}$ & $\begin{array}{l}150^{\circ} \mathrm{C}, \mathrm{Mg} \text { foil, } \\
10 \mathrm{~mol} \% \\
\mathrm{Mg}(\mathrm{TFSA})_{2} \text { in } 90 \\
\mathrm{~mol} \% \mathrm{CsTFSA}\end{array}$ & $\begin{array}{l}\text { 3:1 volume ratio } \\
\text { of Rocksalt } \\
\text { formation (CoO } \\
\text { and } \mathrm{MnO} \text { ) and } \\
\text { spinel phase } \\
\text { (lattice expansion } \\
\text { observed with } \mathrm{Mg} \\
\text { intercalation) on } \\
\text { discharge. } \\
\text { Restoration of the } \\
\text { spinel structure } \\
\text { on charge (XRD } \\
\text { and Rietveld } \\
\text { refinement) }\end{array}$ & $\begin{array}{l}\text { Reduction from } \\
\mathrm{Co}^{3+} \text { to } \mathrm{Co}^{2+} \text { on } \\
\text { discharge, } \\
\text { oxidation to } \mathrm{Co}^{3+} \\
\text { on charge } \\
\text { (XANES) }\end{array}$ & $\begin{array}{l}165 \mathrm{~mA} \mathrm{~h} \mathrm{~g}^{-1} \\
\text { (discharge) } \\
175 \mathrm{~mA} \mathrm{~h} \mathrm{~g}{ }^{-1} \\
\text { (charge) }\end{array}$ & $\begin{array}{l}\text { Mg insertion on } \\
\text { discharge } \\
\text { confirmed (data } \\
\text { not presented) } \\
\text { (EDX) }\end{array}$ & S6 \\
\hline $\mathrm{MgCO}_{2} \mathrm{O}_{4}$ & $\begin{array}{l}100^{\circ} \mathrm{C}, \mathrm{AZ31}, 0.1 \\
\mathrm{M} \mathrm{Mg}(\mathrm{TFSA})_{2} \text { in } \\
\text { triglyme }\end{array}$ & Not provided & Not provided & $\begin{array}{l}165 \mathrm{~mA} \mathrm{~h} \mathrm{~g}^{-1} \\
\text { (discharge) }\end{array}$ & Not provided & S7 \\
\hline
\end{tabular}




\begin{tabular}{|c|c|c|c|c|c|c|}
\hline Material & $\begin{array}{c}\text { Cycling } \\
\text { conditions: } \\
\text { Temperature, } \\
\text { anode, and } \\
\text { electrolyte } \\
\end{array}$ & $\begin{array}{l}\text { Magnesiated } \\
\text { Product phases }\end{array}$ & Redox changes & $\begin{array}{c}\text { Reported } \\
\text { Electrochemical } \\
\text { Capacity }\end{array}$ & $\begin{array}{l}\text { Experimentally } \\
\text { Verified } \\
\text { Magnesiated } \\
\text { Composition }\end{array}$ & Reference \\
\hline $\mathrm{MgCo}_{2} \mathrm{O}_{4}$ & $\begin{array}{l}\text { Room } \\
\text { temperature, } \mathrm{Mg} \\
\text { foil, } 1.0 \mathrm{M} \\
\mathrm{MgTFSI}_{2} \text { in } \\
\text { diglyme }\end{array}$ & $\begin{array}{l}\text { Removal of Mg } \\
\text { from spinel } \\
\text { lattice, formation } \\
\text { of an amorphous } \\
\text { MgO phase at } \\
\text { particle surfaces } \\
\text { (EDS/TEM) }\end{array}$ & $\begin{array}{l}\text { Oxidation of } \mathrm{Co}^{3+} \\
\text { to } \mathrm{Co}^{4+} \text { at } \\
\text { particle surfaces } \\
\text { on charge (EELS) }\end{array}$ & $\begin{array}{l}20 \mathrm{~mA} \mathrm{~h} \mathrm{~g}^{-1} \text { (first } \\
\text { charge), } 40 \mathrm{~mA} \mathrm{~h} \\
\mathrm{~g}^{-1} \text { (first } \\
\text { discharge), } 75 \\
\mathrm{~mA} \mathrm{~h} \mathrm{~g}^{-1} \text { (cycle 6) }\end{array}$ & Not provided & S8 \\
\hline $\mathrm{ZnCo}_{2} \mathrm{O}_{4}$ & $\begin{array}{l}150^{\circ} \mathrm{C}, \mathrm{Mg} \text { foil, } \\
10 \text { mol\% } \\
\mathrm{Mg}(\mathrm{TFSA})_{2} \text { in } 90 \\
\text { mol\% CsTFSA }\end{array}$ & $\begin{array}{l}\text { Reversible } \\
\text { formation of } \\
\mathrm{MgO}, \mathrm{CoO} \\
\text { rocksalt phases } \\
\text { formed on } \\
\text { discharge/charge. }\end{array}$ & $\begin{array}{l}\text { Reduction of } \\
\mathrm{Co}^{3+} \text { to } \mathrm{Co}^{2+} \text { on } \\
\text { discharge, } \mathrm{Co}^{2} \\
\text { oxidized to } \mathrm{Co}^{3+} \\
\text { on charge } \\
\text { (XANES) }\end{array}$ & $\begin{array}{l}90 \mathrm{~mA} \mathrm{~h} \mathrm{~g}^{-1} \text { (first } \\
\text { discharge), } 60 \mathrm{~mA} \\
\mathrm{~h} \mathrm{~g}^{-1} \text { (cycle } 10 \text { ) }\end{array}$ & $\begin{array}{l}\mathrm{Mg}_{0.55} \mathrm{Zn}_{0.96} \mathrm{Co}_{2} \mathrm{O}_{4} \\
\text { (discharge) } \\
\mathrm{Mg}_{0.16} \mathrm{Zn}_{0.86} \mathrm{Co}_{2} \mathrm{O}_{4} \\
\text { (charge) (EDX) }\end{array}$ & S9 \\
\hline $\mathrm{MgCO}_{2} \mathrm{O}_{4}$ & $\begin{array}{l}70^{\circ} \mathrm{C} \text {, capacitive } \\
\text { carbon, } 1 \mathrm{M} \\
\mathrm{Mg}(\mathrm{TFSA})_{2} \text { in } \\
\text { adiponitrile }\end{array}$ & $\begin{array}{l}\text { Formation of } \\
\text { rocksalt phases } \\
\text { on discharge } \\
\text { (TEM) }\end{array}$ & Not provided & $\begin{array}{l}45 \mathrm{~mA} \mathrm{~h} \mathrm{~g}^{-1} \text { (first } \\
\text { charge), } 125 \mathrm{~mA} \\
\mathrm{~h} \mathrm{~g}^{-1} \text { (first } \\
\text { discharge), } 110 \\
\mathrm{~mA} \mathrm{~h} \mathrm{~g}^{-1} \text { (cycle 4) }\end{array}$ & Not provided & S10 \\
\hline
\end{tabular}


Cobalt Oxides

\begin{tabular}{|c|c|c|c|c|c|c|}
\hline Material & $\begin{array}{c}\text { Cycling } \\
\text { conditions: } \\
\text { Temperature, } \\
\text { anode, and } \\
\text { electrolyte }\end{array}$ & $\begin{array}{l}\text { Magnesiated } \\
\text { Product phases }\end{array}$ & Redox changes & $\begin{array}{c}\text { Reported } \\
\text { Electrochemical } \\
\text { Capacity }\end{array}$ & $\begin{array}{l}\text { Experimentally } \\
\text { Verified } \\
\text { Magnesiated } \\
\text { Composition }\end{array}$ & Reference \\
\hline $\begin{array}{l}\left.\text { a) } \mathrm{MgCo}_{2} \mathrm{O}_{4}, \mathrm{~b}\right) \\
\mathrm{MgCo}_{1.6} \mathrm{Mn}_{0.4} \mathrm{O}_{4}\end{array}$ & $\begin{array}{l}\left.\text { c) } 60{ }^{\circ} \mathrm{C}, \mathrm{d}\right) 90^{\circ} \mathrm{C} \text {, } \\
\mathrm{AZ3} 1 \text { alloy, } 1 \mathrm{M} \\
\mathrm{Mg}(\mathrm{TFSI})_{2} \text { in } \\
\text { triglyme }\end{array}$ & Not provided & Not provided & 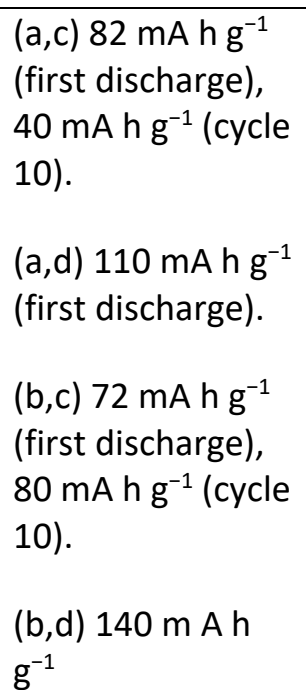 & Not provided & S11,12 \\
\hline $\mathrm{Mg}_{1.04} \mathrm{Co}_{1.36} \mathrm{Mn}_{0.6} \mathrm{O}_{4}$ & $\begin{array}{l}90^{\circ} \mathrm{C}, \mathrm{AZ31} \text { alloy, } \\
1 \mathrm{M} \mathrm{Mg}(\mathrm{TFSI})_{2} \text { in } \\
\text { triglyme }\end{array}$ & $\begin{array}{l}\text { Formation } \\
\text { majority rock salt } \\
\text { phase ( } 98 \text { vol\%) } \\
\text { and expansion of } \\
\text { the remaining } \\
\text { spinel unit cell ( } 2 \\
\text { vol\%) on } \\
\text { discharge (XRD) }\end{array}$ & $\begin{array}{l}\text { Reduction of } \\
\mathrm{Co}^{2.67+} \text { to } \mathrm{Co}^{2+} \\
\text { and } \mathrm{Mn}^{4+} \text { to } \\
\mathrm{Mn}^{3+} \text { on } \\
\text { discharge, both } \\
\text { species re- } \\
\text { oxidized on } \\
\text { charge (XANES) }\end{array}$ & 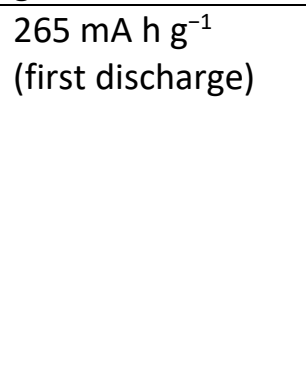 & $\begin{array}{l}\mathrm{Mg}_{2.04} \mathrm{Co}_{1.44} \mathrm{Mn}_{0.56} \mathrm{O}_{4} \\
\text { (discharge, Rietveld } \\
\text { refinement) }\end{array}$ & S13 \\
\hline
\end{tabular}


Cobalt Oxides

\begin{tabular}{|c|c|c|c|c|c|c|}
\hline Material & $\begin{array}{c}\text { Cycling } \\
\text { conditions: } \\
\text { Temperature, } \\
\text { anode, and } \\
\text { electrolyte }\end{array}$ & $\begin{array}{l}\text { Magnesiated } \\
\text { Product phases }\end{array}$ & Redox changes & $\begin{array}{l}\text { Reported } \\
\text { Electrochemical } \\
\text { Capacity }\end{array}$ & $\begin{array}{c}\text { Experimentally } \\
\text { Verified } \\
\text { Magnesiated } \\
\text { Composition }\end{array}$ & Reference \\
\hline $\mathrm{Co}_{3} \mathrm{O}_{4}$ & $\begin{array}{l}\text { Room } \\
\text { temperature, } \mathrm{Mg} \\
\text { foil, } 1 \mathrm{M} \\
\mathrm{Mg}\left(\mathrm{ClO}_{4}\right)_{2} \text { in } \\
\text { acetonitrile with } \\
\text { a) no water } \\
\text { added and b) } 1 \mathrm{M} \\
\text { water added }\end{array}$ & Not provided & Not provided & $\begin{array}{l}\text { a) No } \\
\text { electrochemical } \\
\text { activity observed } \\
\text { b) Irreversible } \\
\text { reduction of the } \\
\text { electrode } \\
\text { (capacity not } \\
\text { quantified) }\end{array}$ & Not provided & S14 \\
\hline $\mathrm{MgCoSiO}_{4}$ & $\begin{array}{l}\text { Room } \\
\text { temperature, } \mathrm{Mg} \\
\text { foil, } 0.25 \mathrm{M} \\
\mathrm{Mg}\left(\mathrm{AlCl}{ }_{2} \mathrm{EtBu}\right)_{2} \text { in } \\
\mathrm{THF}\end{array}$ & Not provided & Not provided & $\begin{array}{l}200 \mathrm{~mA} \mathrm{~h} \mathrm{~g}^{-1} \\
\text { (first discharge), } \\
110 \mathrm{~mA} \mathrm{~h} \mathrm{~g}^{-1} \\
\text { (cycle 200) }\end{array}$ & Not provided & S15 \\
\hline $\mathrm{MgCoSiO}_{4}$ & $\begin{array}{l}\text { Room } \\
\text { temperature, } \mathrm{Mg} \\
\text { foil, } 0.25 \mathrm{M} \\
\mathrm{Mg}\left(\mathrm{AlCl}{ }_{2} \mathrm{EtBu}\right)_{2} \text { in } \\
\mathrm{THF}\end{array}$ & $\begin{array}{l}\text { No changes to } \\
\text { unit cell } \\
\text { parameters } \\
\text { observed (XRD) }\end{array}$ & Not provided & $\begin{array}{l}125 \mathrm{~mA} \mathrm{~h} \mathrm{~g}^{-1} \\
\text { (first discharge), } \\
160 \mathrm{~mA} \mathrm{~h} \mathrm{~g}^{-1} \\
\text { (cycle 7) }\end{array}$ & $\begin{array}{l}\mathrm{Mg}_{0.34} \mathrm{CoSiO}_{4} \\
\text { (charged), } \\
\mathrm{Mg}_{0.64} \mathrm{CoSiO}_{4} \\
\text { (discharged) (ICP- } \\
\text { AES) }\end{array}$ & S16 \\
\hline $\mathrm{MgCoSiO}_{4}$ & $\begin{array}{l}24{ }^{\circ} \mathrm{C}, \mathrm{Mg} \text { alloy } \\
\mathrm{AZ61}, 0.4 \mathrm{M} \mathrm{APC} \\
\text { electrolyte } \\
(\mathrm{PhMgCl} \text { and } \\
\mathrm{AlCl}_{3} \text { in } 2: 1 \text { molar } \\
\text { ratio) in THF }\end{array}$ & Not provided & Not provided & $\begin{array}{l}9 \mathrm{~mA} \mathrm{~h} \mathrm{~g}^{-1} \text { (first } \\
\text { discharge), } 4 \mathrm{~mA} \\
\mathrm{~h} \mathrm{~g}^{-1} \text { (long-term } \\
\text { cycling) }\end{array}$ & Not provided & S17 \\
\hline
\end{tabular}


Iron Oxides/Phosphates

\begin{tabular}{|c|c|c|c|c|c|c|}
\hline Material & $\begin{array}{c}\text { Cycling } \\
\text { conditions: } \\
\text { Temperature, } \\
\text { anode, and } \\
\text { electrolyte } \\
\end{array}$ & $\begin{array}{l}\text { Magnesiated } \\
\text { Product phases }\end{array}$ & Redox changes & $\begin{array}{c}\text { Reported } \\
\text { Electrochemical } \\
\text { Capacity }\end{array}$ & $\begin{array}{l}\text { Experimentally } \\
\text { Verified } \\
\text { Magnesiated } \\
\text { Composition }\end{array}$ & Reference \\
\hline $\mathrm{MgFe}_{2} \mathrm{O}_{4}$ & $\begin{array}{l}150{ }^{\circ} \mathrm{C}, \mathrm{Mg} \text { foil, } \\
0.45 \mathrm{M} \text { CsTFSA + } \\
0.05 \mathrm{M} \mathrm{Mg(TFSA)}\end{array}$ & $\begin{array}{l}\mathrm{FeO} \text { and } \mathrm{MgO} \\
\text { formation on } \\
\text { discharge (XRD) }\end{array}$ & $\begin{array}{l}\text { Minor reduction } \\
\text { of } \mathrm{Fe}^{3+} \text { to } \mathrm{Fe}^{2+} \\
\text { (XANES) }\end{array}$ & $\begin{array}{l}235 \mathrm{~mA} \mathrm{~h} \mathrm{~g}^{-1} \text { (first } \\
\text { discharge) }\end{array}$ & Not provided & S6 \\
\hline $\mathrm{ZnFe}_{2} \mathrm{O}_{4}$ & $\begin{array}{l}150^{\circ} \mathrm{C}, \mathrm{Mg} \text { foil, } 10 \\
\mathrm{~mol} \% \mathrm{Mg}(\mathrm{TFSA})_{2} \\
\text { in } 90 \mathrm{~mol} \% \\
\mathrm{CsTFSA}\end{array}$ & $\begin{array}{l}\text { Rocksalt FeO and } \\
\mathrm{MgO} \text { phase } \\
\text { formation with } \\
\text { discharge, spinel } \\
\text { structure returns } \\
\text { on charge (XRD) }\end{array}$ & Not provided & $\begin{array}{l}145 \mathrm{~mA} \mathrm{~h} \mathrm{~g}^{-1} \text { (first } \\
\text { discharge), } 80 \mathrm{~mA} \\
\mathrm{~h} \mathrm{~g}^{-1} \text { (cycle 10) }\end{array}$ & $\begin{array}{l}\mathrm{Mg}_{0.73} \mathrm{Zn}_{0.97} \mathrm{Fe}_{2} \mathrm{O}_{4} \\
\text { (discharged) } \\
\mathrm{Mg}_{0.22} \mathrm{Zn}_{0.88} \mathrm{Fe}_{2} \mathrm{O}_{4} \\
\text { (charged) (EDX) }\end{array}$ & S9 \\
\hline $\mathrm{MgFeSiO}_{4}$ & $\begin{array}{l}\text { Room } \\
\text { temperature, } \mathrm{Mg} \\
\text { foil, } 0.25 \mathrm{M} \\
\mathrm{Mg}\left(\mathrm{AlCl}_{2} \mathrm{EtBu}\right)_{2} \text { in } \\
\mathrm{THF}\end{array}$ & Not provided & Not provided & $\begin{array}{l}125 \mathrm{~mA} \mathrm{~h} \mathrm{~g}^{-1} \text { (first } \\
\text { cycle), } 110 \mathrm{~mA} \mathrm{~h} \\
\mathrm{~g}^{-1} \text { (cycle } 20 \text { ) }\end{array}$ & Not provided & S18 \\
\hline $\mathrm{MgFeSiO}_{4}$ & $\begin{array}{l}24{ }^{\circ} \mathrm{C}, \mathrm{AZ61}, 0.4 \\
\mathrm{M} \mathrm{APC} \text { electrolyte } \\
\text { (PhMgCl and } \mathrm{AlCl}_{3} \\
\text { in } 2: 1 \text { molar ratio) } \\
\text { in THF }\end{array}$ & Not provided & Not provided & 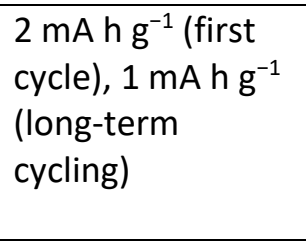 & Not provided & S17 \\
\hline $\mathrm{MgFePO}_{4} \mathrm{~F}$ & $\begin{array}{l}\text { Room } \\
\text { temperature, } \mathrm{Mg} \\
\text { foil, } 0.5 \mathrm{M} \\
\mathrm{Mg}(\mathrm{TFSI})_{2} \text { in } \\
\text { acetonitrile }\end{array}$ & Not provided & Not provided & $\begin{array}{l}35 \mathrm{~mA} \mathrm{~h} \mathrm{~g}^{-1} \text { (first } \\
\text { cycle and cycle } \\
\text { 10) }\end{array}$ & Not provided & S19 \\
\hline
\end{tabular}


Iron Oxides/Phosphates

\begin{tabular}{|c|c|c|c|c|c|c|}
\hline Material & $\begin{array}{c}\text { Cycling } \\
\text { conditions: } \\
\text { Temperature, } \\
\text { anode, and } \\
\text { electrolyte }\end{array}$ & $\begin{array}{l}\text { Magnesiated } \\
\text { Product phases }\end{array}$ & Redox changes & $\begin{array}{c}\text { Reported } \\
\text { Electrochemical } \\
\text { Capacity }\end{array}$ & $\begin{array}{c}\text { Experimentally } \\
\text { Verified } \\
\text { Magnesiated } \\
\text { Composition }\end{array}$ & Reference \\
\hline $\mathrm{FePO}_{4}$ (defective) & $\begin{array}{l}25^{\circ} \mathrm{C} \text {, capacitive } \\
\text { carbon, } 0.5 \mathrm{M} \\
\mathrm{Mg}(\mathrm{TFSA})_{2} \text { in } \\
\text { acetonitrile } \\
\text { (electrolyte water } \\
\text { content }<300 \\
\text { ppm). }\end{array}$ & $\begin{array}{l}\text { Changes observed } \\
\text { consistent with } \\
\text { Mg insertion } \\
\text { (EXAFS) }\end{array}$ & $\begin{array}{l}\text { Partial reduction } \\
\text { of } \mathrm{Fe}^{3+} \text { to } \mathrm{Fe}^{2+} \\
\text { with discharge, } \\
\text { incomplete re- } \\
\text { oxidation of } \mathrm{Fe}^{2+} \\
\text { to } \mathrm{Fe}^{3+} \text { on charge } \\
\text { (XANES) }\end{array}$ & $\begin{array}{l}180 \mathrm{~mA} \mathrm{~h} \mathrm{~g}{ }^{-1} \text { (first } \\
\text { cycle), } 140 \mathrm{~mA} \mathrm{~h} \\
\mathrm{~g}^{-1} \text { (cycle } 20 \text { ) }\end{array}$ & $\begin{array}{l}\mathrm{Mg}_{0.33} \mathrm{FePO}_{4} \\
\text { (EELS) } \\
\mathrm{Mg}_{0.22} \mathrm{FePO}_{4} \\
\text { (XANES) } \\
\text { (discharged) }\end{array}$ & S20 \\
\hline $\mathrm{FePO}_{4}$ & $\begin{array}{l}55^{\circ} \mathrm{C}, \mathrm{Mg} \text { foil, } 0.5 \\
\mathrm{M} \mathrm{Mg}(\mathrm{TFSI})_{2} \text { in } \\
\text { acetonitrile. }\end{array}$ & $\begin{array}{l}\text { Expansion of the } \\
\text { FePO }_{4} \text { lattice with } \\
\text { discharge, } \\
\text { contraction with } \\
\text { charge (XRD) }\end{array}$ & $\begin{array}{l}\text { Partial reduction } \\
\text { of } \mathrm{Fe}^{3+} \text { to } \mathrm{Fe}^{2+} \\
\text { with discharge, } \\
\text { incomplete re- } \\
\text { oxidation of } \mathrm{Fe}^{2+} \\
\text { to } \mathrm{Fe}^{3+} \text { on charge } \\
\text { (XANES) }\end{array}$ & $\begin{array}{l}150 \mathrm{~mA} \mathrm{~h} \mathrm{~g}^{-1} \text { (first } \\
\text { discharge), } 110 \\
\mathrm{~mA} \mathrm{~h}^{-1} \text { (second } \\
\text { discharge) }\end{array}$ & Not provided & S21 \\
\hline $\mathrm{FePO}_{4}$ & $\begin{array}{l}\text { Room } \\
\text { temperature, } \mathrm{Ag} \\
\text { foil, } 0.5 \mathrm{M} \\
\mathrm{Mg}\left(\mathrm{ClO}_{4}\right)_{2} \text { in } \\
\text { water }\end{array}$ & Not provided & Not provided & $\begin{array}{l}122 \mathrm{~mA} \mathrm{~h} \mathrm{~g}^{-1} \text { (first } \\
\text { discharge) }\end{array}$ & Not provided & S22 \\
\hline $\mathrm{FePO}_{4}$ & $\begin{array}{l}\text { Room } \\
\text { temperature, } \mathrm{Pt} \\
\text { foil, } 0.5 \mathrm{M} \mathrm{MgSO}_{4} \\
\text { in water }\end{array}$ & $\begin{array}{l}\text { Peak broadening } \\
\text { with discharge, } \\
\text { reversed on } \\
\text { charge. Slight } \\
\text { lattice shifts } \\
\text { observed (XRD) }\end{array}$ & $\begin{array}{l}50 \% \text { conversion } \\
\text { of } \mathrm{Fe}^{3+} \text { to } \mathrm{Fe}^{2+} \text { on } \\
\text { discharge, } \\
\text { partially reversed } \\
\text { on charge } \\
\text { (Mossbauer) }\end{array}$ & $\begin{array}{l}95 \mathrm{~mA} \mathrm{~h} \mathrm{~g}^{-1} \text { (first } \\
\text { discharge), } 65 \mathrm{~mA} \\
\mathrm{~h} \mathrm{~g}^{-1} \text { (cycle 3) }\end{array}$ & $\begin{array}{l}\mathrm{Mg}_{0.38} \mathrm{FePO}_{4} \\
\text { (discharged) } \\
\mathrm{Mg}_{0.02} \mathrm{FePO}_{4} \\
\text { (charged) (ICP- } \\
\text { AES) }\end{array}$ & S23 \\
\hline
\end{tabular}


Iron Oxides/Phosphates

\begin{tabular}{|c|c|c|c|c|c|c|}
\hline Material & $\begin{array}{c}\text { Cycling } \\
\text { conditions: } \\
\text { Temperature, } \\
\text { anode, and } \\
\text { electrolyte }\end{array}$ & $\begin{array}{l}\text { Magnesiated } \\
\text { Product phases }\end{array}$ & Redox changes & $\begin{array}{c}\text { Reported } \\
\text { Electrochemical } \\
\text { Capacity }\end{array}$ & $\begin{array}{c}\text { Experimentally } \\
\text { Verified } \\
\text { Magnesiated } \\
\text { Composition }\end{array}$ & Reference \\
\hline $\mathrm{FePO}_{4}$ & $\begin{array}{l}\text { Room } \\
\text { temperature, } \mathrm{Mg} \\
\text { foil, a) } 0.3 \mathrm{M} \\
\text { magnesium } \\
\text { monocarborane } \\
\text { in tetraglyme b) } \\
0.4 \mathrm{M} \mathrm{APC} \\
\text { electrolyte } \\
\text { (PhMgCl and } \mathrm{AlCl}_{3} \\
\text { in } 2: 1 \text { molar ratio) } \\
\text { in THF }\end{array}$ & $\begin{array}{l}\text { a) No significant } \\
\text { crystallographic } \\
\text { differences on } \\
\text { discharge (XRD). } \\
\text { Amorphization of } \\
\text { 3-5 nm thick } \\
\text { surface layer } \\
\text { FePO }{ }_{4} \text { on } \\
\text { discharge (ATR- } \\
\text { FTIR and TEM) }\end{array}$ & Not provided & 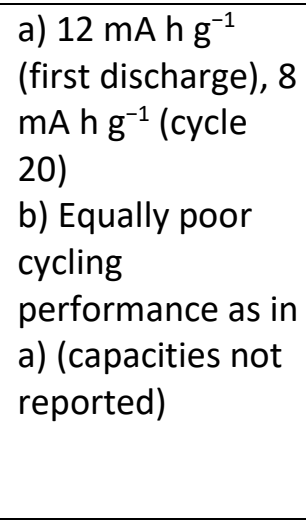 & $\begin{array}{l}\text { a) } \sim \mathrm{Mg}_{0.2} \mathrm{FePO}_{4} \text { at } \\
\text { the surface, } \\
\mathrm{FePO}_{4} \text { in the bulk } \\
\text { (XPS depth } \\
\text { profiling) }\end{array}$ & S24 \\
\hline
\end{tabular}




\begin{tabular}{|c|c|c|c|c|c|c|}
\hline Material & $\begin{array}{c}\text { Cycling } \\
\text { conditions: } \\
\text { Temperature, } \\
\text { anode, and } \\
\text { electrolyte }\end{array}$ & $\begin{array}{l}\text { Magnesiated } \\
\text { Product phases }\end{array}$ & Redox changes & $\begin{array}{c}\text { Reported } \\
\text { Electrochemical } \\
\text { Capacity }\end{array}$ & $\begin{array}{l}\text { Experimentally } \\
\text { Verified } \\
\text { Magnesiated } \\
\text { Composition }\end{array}$ & Reference \\
\hline $\begin{array}{l}\mathrm{MnO}_{2} \text { (phase not } \\
\text { given) }\end{array}$ & $\begin{array}{l}\text { Room } \\
\text { temperature, } \mathrm{Pt} \\
\text { foil, a) } 0.1 \mathrm{M} \\
\left.\mathrm{Mg}\left(\mathrm{ClO}_{4}\right)_{2} \text { in } \mathrm{PC}, \mathrm{b}\right) \\
0.1 \mathrm{M} \\
\mathrm{Mg}\left(\mathrm{ClO}_{4}\right)_{2} \cdot 6 \mathrm{H}_{2} \mathrm{O} \text { in } \\
\mathrm{PC} \text {. }\end{array}$ & $\begin{array}{l}\text { b) Co-intercalation } \\
\text { of } \sim 3 \text { water } \\
\text { molecules with } \mathrm{Mg} \\
\text { (EQCM). Surface } \\
\text { enrichment of } \mathrm{Mg} \\
\text { compared to bulk } \\
\text { (TEM/EDS). }\end{array}$ & Not provided & $\begin{array}{l}\text { b) } 145 \mathrm{~mA} \mathrm{~h} \mathrm{~g}^{-1} \\
\text { (first cycle), } 110 \\
\mathrm{~mA} \mathrm{~h} \mathrm{~g}^{-1} \text { (cycle } \\
\text { 200) }\end{array}$ & $\begin{array}{l}\left.\text { a) } \mathrm{Mg}_{0.18} \mathrm{MnO}_{2}, \mathrm{~b}\right) \\
\mathrm{Mg}_{0.69} \mathrm{MnO}_{2}(\mathrm{ICP}- \\
\text { AES) }\end{array}$ & S25 \\
\hline $\begin{array}{l}\mathrm{MnO}_{2} \text { (phase not } \\
\text { given) }\end{array}$ & $\begin{array}{l}\text { Room } \\
\text { temperature, } \mathrm{Pt} \\
\text { foil, a) } 0.1 \mathrm{M} \\
\mathrm{Mg}\left(\mathrm{ClO}_{4}\right)_{2} \text { in } \mathrm{PC} \text {, b) } \\
0.1 \mathrm{M} \\
\mathrm{Mg}\left(\mathrm{ClO}_{4}\right)_{2} \cdot 6 \mathrm{H}_{2} \mathrm{O} \text { in } \\
\mathrm{PC} \text {. }\end{array}$ & $\begin{array}{l}\text { b) Surface } \\
\mathrm{Mg}(\mathrm{OH})_{2} \text { phases }\end{array}$ & Not provided & Not provided & $\begin{array}{l}\text { b) } \mathrm{Mg}_{0.25} \mathrm{Mn}_{2} \mathrm{O}_{4} \\
\text { (discharged), } \\
\mathrm{Mg}_{0.1} \mathrm{Mn}_{2} \mathrm{O}_{4} \\
\text { (charged) (XPS } \\
\text { depth profiling). } \\
\text { The surface had } \\
\text { greater } \mathrm{Mg} \\
\text { enrichment. }\end{array}$ & S26 \\
\hline $\begin{array}{l}\text { Birnessite- } \delta- \\
\mathrm{MnO}_{2}\end{array}$ & $\begin{array}{l}20{ }^{\circ} \mathrm{C}, \mathrm{Mg} \text { foil, } 1 \mathrm{M} \\
\text { of } \mathrm{Mg}\left(\mathrm{ClO}_{4}\right)_{2} \text { in } \\
\text { acetonitrile }\end{array}$ & Not provided & Not provided & $\begin{array}{l}105 \mathrm{~mA} \mathrm{~h} \mathrm{~g}^{-1} \text { (first } \\
\text { cycle) } \\
60 \mathrm{~mA} \mathrm{~h} \mathrm{~g}^{-1} \text { (cycle } \\
25 \text { ) }\end{array}$ & Not provided & S27 \\
\hline $\begin{array}{l}\text { Birnessite }-\delta- \\
\mathrm{MnO}_{2} \\
\left(\mathrm{Mg}_{0.15} \mathrm{MnO}_{2} \cdot 0.9 \mathrm{H}_{2}\right. \\
\text { O) }\end{array}$ & $\begin{array}{l}20{ }^{\circ} \mathrm{C} \text {, capacitive } \\
\text { carbon, } 0.25 \mathrm{M} \\
\mathrm{Mg}(\mathrm{TFSI})_{2} / \text { diglyme }\end{array}$ & $\begin{array}{l}\text { Broadened (001) } \\
\text { peak (the layer } \\
\text { axis) with cycling } \\
(\mathrm{XRD}), \mathrm{MnO}, \\
\mathrm{MnOOH}, \mathrm{Mg}(\mathrm{OH})_{2} \\
\text { surface phases } \\
\text { (XPS) }\end{array}$ & $\begin{array}{l}\text { Partial reduction } \\
\text { of } \mathrm{Mn}^{4+} \text { to } \mathrm{Mn}^{3+} \text { in } \\
\text { the bulk, with } \\
\text { over-reduction to } \\
\mathrm{Mn}^{2+} \text { at the } \\
\text { surface, with } \\
\text { discharge. Only } \\
\text { partially reversed } \\
\text { on charge. (XPS, } \\
\text { EELS) }\end{array}$ & $\begin{array}{l}130 \mathrm{~mA} \mathrm{~h} \mathrm{~g}^{-1} \text { (first } \\
\text { cycle) } \\
135 \mathrm{~mA} \mathrm{~h} \mathrm{~g}^{-1} \\
\text { (cycle 50) }\end{array}$ & Not provided & S28 \\
\hline
\end{tabular}


Manganese Oxides

\begin{tabular}{|c|c|c|c|c|c|c|}
\hline Material & $\begin{array}{c}\text { Cycling } \\
\text { conditions: } \\
\text { Temperature, } \\
\text { anode, and } \\
\text { electrolyte }\end{array}$ & $\begin{array}{l}\text { Magnesiated } \\
\text { Product phases }\end{array}$ & Redox changes & $\begin{array}{c}\text { Reported } \\
\text { Electrochemical } \\
\text { Capacity }\end{array}$ & $\begin{array}{c}\text { Experimentally } \\
\text { Verified } \\
\text { Magnesiated } \\
\text { Composition }\end{array}$ & Reference \\
\hline $\begin{array}{l}\text { Birnessite - } \\
\delta-\mathrm{MnO}_{2} \\
\text { a) } \mathrm{Mg}_{0.15} \\
\mathrm{Na}_{0.02} \mathrm{MnO}_{2} \cdot 0.7 \mathrm{H}_{2} \mathrm{O} \\
\text { b) } \\
\mathrm{Mg}_{0.14} \mathrm{Na}_{0.04} \mathrm{MnO}_{2} . \\
0.7 \mathrm{H}_{2} \mathrm{O}\end{array}$ & $\begin{array}{l}0.4 \mathrm{M} \mathrm{Mg}(\mathrm{TFSI})_{2} \\
\text { with } 2.4 \mathrm{M} \text { water } \\
\text { and } 0.5 \mathrm{M} \text { dipro- } \\
\text { glyme in } \\
\text { acetonitrile }\end{array}$ & Not provided & Not provided & $\begin{array}{l}\text { a) } 138 \mathrm{~mA} \mathrm{~h} \mathrm{~g}{ }^{-1} \\
\text { (first cycle), } 115 \\
\mathrm{~mA} \mathrm{~h} \mathrm{~g}{ }^{-1} \text { (cycle 5) } \\
\text { b) } 80 \mathrm{~mA} \mathrm{~h} \mathrm{~g}^{-1} \\
\text { (first cycle), } 95 \mathrm{~mA} \\
\mathrm{~h} \mathrm{~g}^{-1} \text { (cycle 5) }\end{array}$ & Not provided & S29 \\
\hline $\begin{array}{l}\text { Birnessite }-\delta- \\
\mathrm{MnO}_{2} \cdot 0.94 \mathrm{H}_{2} \mathrm{O}\end{array}$ & $\begin{array}{l}\text { Room } \\
\text { temperature, } \mathrm{Mg} \\
\text { foil, } 0.5 \mathrm{M} \\
\mathrm{Mg}\left(\mathrm{ClO}_{4}\right)_{2} \text { in } \\
\text { acetonitrile in a) } \\
\text { dry conditions or } \\
\text { b) } 10 \mathrm{M} \text { water } \\
\text { added }\end{array}$ & $\begin{array}{l}\text { a) Poor } \\
\text { reversibility of } \\
\text { structural changes } \\
\text { with cycling } \\
\text { b) Reversible } \\
\text { disappearance and } \\
\text { re-emergence of } \\
\text { birnessite peaks } \\
\text { with cycling (XRD). } \\
\text { Changes in the } \\
\text { birnessite layered } \\
\text { structure observed } \\
\text { on discharge (ABF- } \\
\text { STEM) }\end{array}$ & $\begin{array}{l}\text { b) Reversible } \\
\text { oxidation state } \\
\text { changes between } \\
\mathrm{Mn}^{4+} \text { and } \mathrm{Mn}^{3+} \\
\text { (XAS, XPS) }\end{array}$ & $\begin{array}{l}\text { a) } 55 \mathrm{~mA} \mathrm{~h} \mathrm{~g}-1 \\
\text { (first cycle), } 25 \mathrm{~mA} \\
\mathrm{~h} \mathrm{~g}^{-1} \text { (cycle 30) } \\
\text { b) } 225 \mathrm{~mA} \mathrm{~h} \mathrm{~g}^{-1} \\
\text { (first cycle), } 190 \\
\mathrm{~mA} \mathrm{~h} \mathrm{~g}{ }^{-1} \text { (cycle 30) }\end{array}$ & $\begin{array}{l}\text { a) } \\
\mathrm{Mg}_{0.12} \mathrm{MnO}_{2} \cdot 1.03 \\
\mathrm{H}_{2} \mathrm{O} \text { (discharge), } \\
\mathrm{MnO}_{2} \cdot 0.91 \mathrm{H}_{2} \mathrm{O} \\
\text { (charge) } \\
\text { b) } \\
\mathrm{Mg}_{0.50} \mathrm{MnO}_{2} \cdot 2.33 \mathrm{H} \\
{ }_{2} \mathrm{O} \text { (discharge), } \\
\mathrm{MnO}_{2} \cdot 0.91 \mathrm{H}_{2} \mathrm{O} \\
\text { (charge) }(\mathrm{ICP}-\mathrm{AES} \\
\text { and TGA) }\end{array}$ & S30 \\
\hline $\begin{array}{l}\text { Birnessite }-\delta- \\
\mathrm{MnO}_{2} \cdot \mathrm{xH}_{2} \mathrm{O}\end{array}$ & $\begin{array}{l}\text { Room } \\
\text { temperature, } \mathrm{Mg} \\
\text { foil, } 0.5 \mathrm{M} \\
\mathrm{Mg}\left(\mathrm{ClO}_{4}\right)_{2} \text { in a) } \\
\text { water and b) } \\
\text { acetonitrile }\end{array}$ & Not provided & Not provided & $\begin{array}{l}\text { a) } 250 \mathrm{~mA} \mathrm{~h} \mathrm{~g}^{-1} \\
\text { (second cycle) } \\
\text { b) } 130 \mathrm{~mA} \mathrm{~h} \mathrm{~g}^{-1} \\
\text { (second cycle) }\end{array}$ & Not provided & S31 \\
\hline
\end{tabular}




\begin{tabular}{|c|c|c|c|c|c|c|}
\hline Material & $\begin{array}{l}\text { Cycling } \\
\text { conditions: } \\
\text { Temperature, } \\
\text { anode, and } \\
\text { electrolyte }\end{array}$ & $\begin{array}{l}\text { Magnesiated } \\
\text { Product phases }\end{array}$ & Redox changes & $\begin{array}{c}\text { Reported } \\
\text { Electrochemical } \\
\text { Capacity }\end{array}$ & $\begin{array}{l}\text { Experimentally } \\
\text { Verified } \\
\text { Magnesiated } \\
\text { Composition }\end{array}$ & Reference \\
\hline $\begin{array}{l}\text { Birnessite- } \delta- \\
\mathrm{MnO}_{2} \cdot 0.08 \mathrm{TMA} \cdot \\
1.24 \mathrm{H}_{2} \mathrm{O}(\mathrm{TMA}= \\
\text { tetramethylammo } \\
\text { nium) }\end{array}$ & $\begin{array}{l}\text { Room } \\
\text { temperature, } \mathrm{Pt} \\
\text { foil, } 1 \mathrm{M} \mathrm{MgSO}_{4} \text { in } \\
\text { water }\end{array}$ & $\begin{array}{l}\text { Co-intercalation of } \\
\sim 3.75 \text { water } \\
\text { molecules with } \\
\mathrm{Mg}^{2+}(\mathrm{EQCM})\end{array}$ & Not provided & $\begin{array}{l}118 \mathrm{~mA} \mathrm{~h} \mathrm{~g}{ }^{-1} \text { (first } \\
\text { cycle), } 100 \mathrm{~mA} \mathrm{~h} \\
\mathrm{~g}^{-1} \text { (cycle 10) }\end{array}$ & Not provided & S32 \\
\hline $\begin{array}{l}\text { Birnessite- } \delta- \\
\mathrm{MnO}_{2} \cdot \mathrm{xH}_{2} \mathrm{O} / \mathrm{Ti}_{3} \mathrm{C}_{2}\end{array}$ & $\begin{array}{l}\text { No information } \\
\text { provided }\end{array}$ & Not provided & Not provided & $\begin{array}{l}175 \mathrm{~mA} \mathrm{~h} \mathrm{~g} \text { (first } \\
\text { cycle), } 100 \mathrm{~mA} \mathrm{~h} \\
\mathrm{~g}^{-1} \text { (cycle } 25 \text { ) }\end{array}$ & Not provided & S33 \\
\hline $\begin{array}{l}\text { Phyllomanganate - } \\
\text { a) } \mathrm{MnO}_{x} \cdot 0.6 \mathrm{H}_{2} \mathrm{O} \text { b) } \\
\mathrm{MnO}_{x} \cdot 0.3 \mathrm{H}_{2} \mathrm{O}\end{array}$ & $\begin{array}{l}\left.\text { c) } 25^{\circ} \mathrm{C}, \mathrm{d}\right) 50{ }^{\circ} \mathrm{C} \text {, } \\
\text { e) } 110{ }^{\circ} \mathrm{C}, \mathrm{Mg} \text { foil, } \\
0.25 \\
\mathrm{M} \mathrm{Mg}\left(\mathrm{CB}_{11} \mathrm{H}_{12}\right)_{2} \\
\text { dissolved in } \\
\text { triglyme }\end{array}$ & $\begin{array}{l}\text { a,c) Largely } \\
\text { reversible } \\
\text { expansion and } \\
\text { contraction of } d \\
\text { spacings with } \\
\text { discharge and } \\
\text { charge. b,d) } \\
\text { Expansion of } d \\
\text { spacings on } \\
\text { discharge, only a } \\
\text { minor reversion on } \\
\text { charge (XRD) } \\
\text { Presence of } \\
\text { intercalated } \\
\text { (structural) } \mathrm{Mg} \\
\text { verified ( }{ }^{25} \mathrm{Mg} \\
\mathrm{NMR} \text { ). }\end{array}$ & $\begin{array}{l}\text { a,c) Reduction of } \\
\mathrm{Mn}^{3+} \text { to } \mathrm{Mn}^{2+} \text { on } \\
\text { discharge, } \\
\text { reoxidized to } \mathrm{Mn}^{3+} \\
\text { on charge. } \mathrm{b}, \mathrm{d} \text { ) } \\
\text { Reduction of } \mathrm{Mn}^{4+} \\
\text { to } \mathrm{Mn}^{2+} \text { on } \\
\text { discharge, further } \\
\text { reduction to } \mathrm{Mn}^{2+} \\
\text { on charge. (XAS) }\end{array}$ & $\begin{array}{l}\text { a,c) } 190 \mathrm{~mA} \mathrm{~h} \mathrm{~g}^{-1} \\
\text { (first discharge). } \\
\text { a,d) } 290 \mathrm{~mA} \mathrm{~h} \mathrm{~g}^{-1} \\
\text { (first discharge). } \\
\text { a,e) } 310 \mathrm{~mA} \mathrm{~h} \mathrm{~g}^{-1} \\
\text { (first discharge). } \\
\text { b,c) } 150 \mathrm{~mA} \mathrm{~h} \mathrm{~g}^{-1} \\
\text { (first discharge). } \\
\text { b,d) } 190 \mathrm{~mA} \mathrm{~h} \mathrm{~g}^{-1} \\
\text { (first discharge) }\end{array}$ & $\begin{array}{l}\text { a,c) } \\
\mathrm{Mg}_{0.38} \mathrm{MnO}_{x} \cdot 0.6 \mathrm{H}_{2} \\
\mathrm{O} \\
\mathrm{b}, \mathrm{d}) \\
\mathrm{Mg}_{0.25} \mathrm{MnO}_{x} \cdot 0.3 \mathrm{H}_{2} \\
\mathrm{O}\end{array}$ & S34 \\
\hline
\end{tabular}




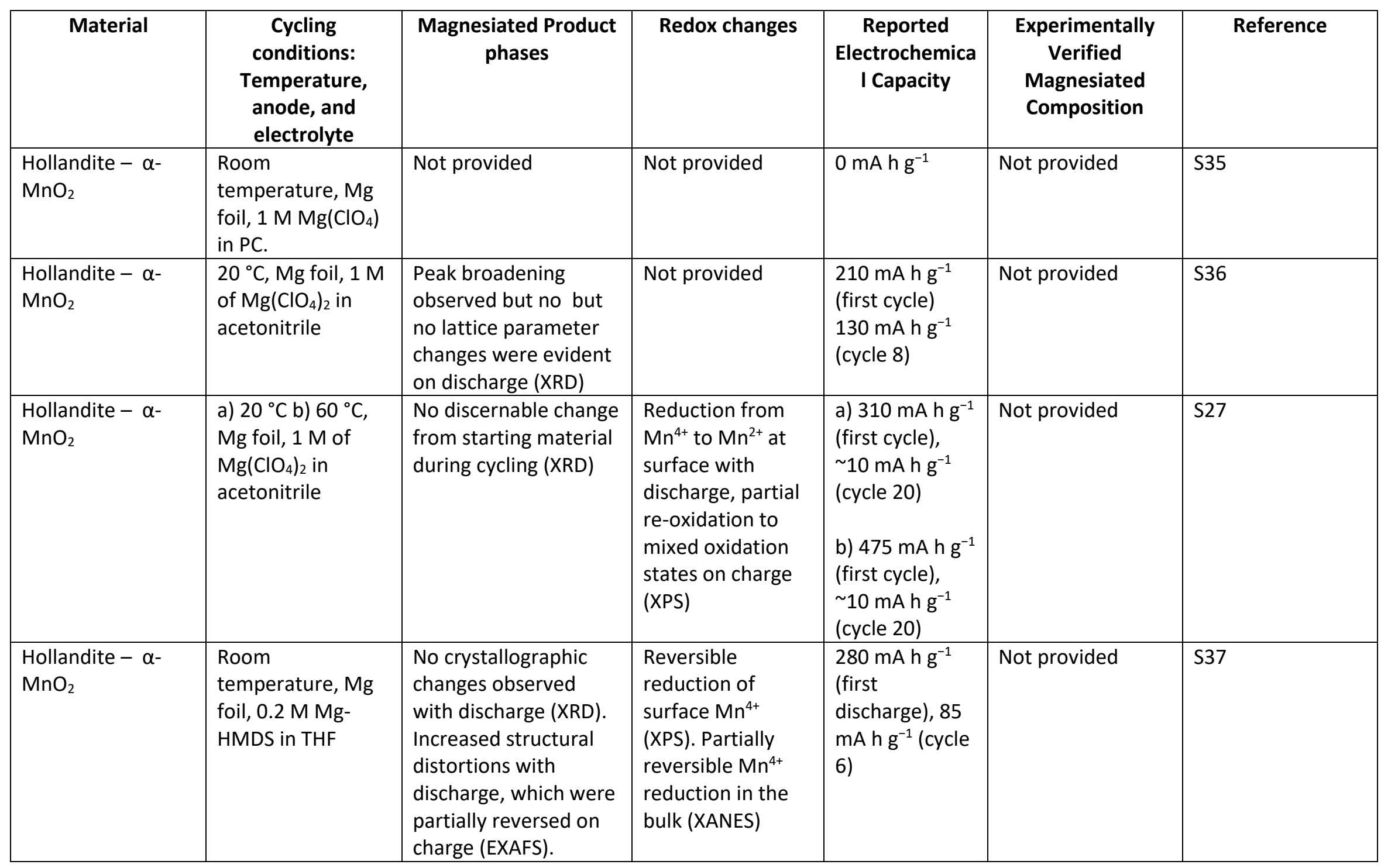




\begin{tabular}{|c|c|c|c|c|c|c|}
\hline Material & $\begin{array}{c}\text { Cycling } \\
\text { conditions: } \\
\text { Temperature, } \\
\text { anode, and } \\
\text { electrolyte }\end{array}$ & $\begin{array}{l}\text { Magnesiated } \\
\text { Product phases }\end{array}$ & Redox changes & $\begin{array}{c}\text { Reported } \\
\text { Electrochemical } \\
\text { Capacity }\end{array}$ & $\begin{array}{l}\text { Experimentally } \\
\text { Verified } \\
\text { Magnesiated } \\
\text { Composition }\end{array}$ & Reference \\
\hline $\begin{array}{l}\text { Hollandite }-\alpha- \\
\mathrm{MnO}_{2}\end{array}$ & $\begin{array}{l}\text { Room } \\
\text { temperature, } \mathrm{Mg} \\
\text { foil, } 0.2 \mathrm{M} \mathrm{APC} \\
\text { electrolyte } \\
\text { ( } \mathrm{PhMgCl} \text { and } \mathrm{AlCl}_{3} \\
\text { in 2:1 molar ratio) } \\
\text { in THF }\end{array}$ & $\begin{array}{l}\text { Conversion } \\
\text { reaction to form } \\
\mathrm{MgO} \text { and } \mathrm{MnO} \\
\text { (HR-TEM) }\end{array}$ & Not provided & $\begin{array}{l}230 \mathrm{~mA} \mathrm{~h} \mathrm{~g}^{-1} \text { (first } \\
\text { discharge), } 70 \mathrm{~mA} \\
\mathrm{~h} \mathrm{~g}^{-1} \text { (cycle 15) }\end{array}$ & $\begin{array}{l}\text { Stoichiometry of } \\
\text { reaction } \\
\text { approximately } \\
\text { verified by } \\
\text { thickness of } \\
\text { MgO/MnO layers } \\
\text { on particle } \\
\text { surfaces (HR-TEM) }\end{array}$ & S38 \\
\hline $\begin{array}{l}3: 1 \alpha-\mathrm{MnO}_{2}: \lambda- \\
\mathrm{MnO}_{2}\end{array}$ & $\begin{array}{l}20{ }^{\circ} \mathrm{C}, \mathrm{Mg} \text { foil, } 0.5 \\
\mathrm{M} \text { of } \mathrm{Mg}\left(\mathrm{ClO}_{4}\right)_{2} \text { in } \\
\text { acetonitrile }\end{array}$ & Not provided & Not provided & 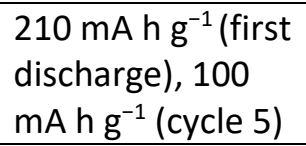 & Not provided & S39 \\
\hline $\begin{array}{l}\text { Todorokite - } \\
\mathrm{MnO}_{2}\end{array}$ & $\begin{array}{l}\text { Room } \\
\text { temperature, } \mathrm{Mg} \\
\text { foil, } 1 \mathrm{M} \mathrm{Mg}\left(\mathrm{ClO}_{4}\right) \\
\text { in } \mathrm{PC}\end{array}$ & Not provided & Not provided & $\begin{array}{l}85 \mathrm{~mA} \mathrm{~h} \mathrm{~g}^{-1} \text { (first } \\
\text { discharge) }\end{array}$ & Not provided & S35 \\
\hline
\end{tabular}


Manganese Oxides

\begin{tabular}{|c|c|c|c|c|c|c|}
\hline Material & $\begin{array}{l}\text { Cycling } \\
\text { conditions: } \\
\text { Temperature, } \\
\text { anode, and } \\
\text { electrolyte }\end{array}$ & $\begin{array}{l}\text { Magnesiated } \\
\text { Product phases }\end{array}$ & Redox changes & $\begin{array}{c}\text { Reported } \\
\text { Electrochemical } \\
\text { Capacity }\end{array}$ & $\begin{array}{l}\text { Experimentally } \\
\text { Verified } \\
\text { Magnesiated } \\
\text { Composition }\end{array}$ & Reference \\
\hline $\begin{array}{l}\text { Ramsdellite - } \\
\mathrm{OMS}^{2} \mathrm{R}-\mathrm{MnO}_{2} \\
\text { molecular sieves }\end{array}$ & $\begin{array}{l}\text { Room } \\
\text { temperature, } \mathrm{Mg} \\
\text { foil, } 1 \mathrm{M} \text { of } \\
\mathrm{Mg}\left(\mathrm{ClO}_{4}\right)_{2} \text { in } \\
\text { acetonitrile }\end{array}$ & Not provided & Not provided & $\begin{array}{l}200 \mathrm{~mA} \mathrm{~h} \mathrm{~g}^{-1} \text { (first } \\
\text { discharge) } \\
50 \mathrm{~mA} \mathrm{~h} \mathrm{~g}^{-1} \text { (cycle } \\
\text { 10) }\end{array}$ & Not provided & S41 \\
\hline $\mathrm{Mg}_{1.03} \mathrm{Mn}_{0.97} \mathrm{SiO}_{4}$ & $\begin{array}{l}\text { Room } \\
\text { temperature, } \mathrm{Mg} \\
\text { foil, } 0.25 \mathrm{M} \\
\mathrm{Mg}\left(\mathrm{AlCl}_{2} \mathrm{EtBu}\right)_{2} / \mathrm{TH} \\
\mathrm{F}\end{array}$ & $\begin{array}{l}\text { No changes } \\
\text { evident with } \\
\text { (XRD) }\end{array}$ & Not provided & $\begin{array}{l}132 \mathrm{~mA} \mathrm{~h} \mathrm{~g}^{-1} \text { (first } \\
\text { charge), } 120 \mathrm{~mA} \mathrm{~h} \\
\mathrm{~g}^{-1} \text { (cycle } 80 \text { ). }\end{array}$ & Not provided & S42 \\
\hline $\mathrm{MgMnSiO}_{4}$ & $\begin{array}{l}25^{\circ} \mathrm{C}, \mathrm{Mg} \text { foil, } 0.25 \\
\mathrm{M} \\
\mathrm{Mg}\left(\mathrm{AlBu}_{2} \mathrm{Cl}_{2}\right)_{2} / \mathrm{THF}\end{array}$ & Not provided & Not provided & 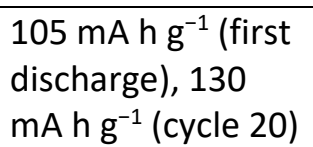 & Not provided & S43 \\
\hline
\end{tabular}


Manganese Oxides

\begin{tabular}{|c|c|c|c|c|c|c|}
\hline Material & $\begin{array}{l}\text { Cycling } \\
\text { conditions: } \\
\text { Temperature, } \\
\text { anode, and } \\
\text { electrolyte }\end{array}$ & $\begin{array}{l}\text { Magnesiated } \\
\text { Product phases }\end{array}$ & Redox changes & $\begin{array}{c}\text { Reported } \\
\text { Electrochemical } \\
\text { Capacity }\end{array}$ & $\begin{array}{l}\text { Experimentally } \\
\text { Verified } \\
\text { Magnesiated } \\
\text { Composition }\end{array}$ & Reference \\
\hline $\begin{array}{l}\mathrm{MWNT} / \mathrm{C} / \mathrm{Mg}_{1.03} \mathrm{M} \\
\mathrm{n}_{0.97} \mathrm{SiO}_{4}\end{array}$ & $\begin{array}{l}\text { Room } \\
\text { temperature, } \mathrm{Mg} \\
\text { foil, } 0.25 \mathrm{M} \\
\mathrm{Mg}\left(\mathrm{AlCl}_{2} \mathrm{EtBu}\right)_{2} / \mathrm{TH} \\
\mathrm{F}\end{array}$ & Not provided & Not provided & $\begin{array}{l}160 \mathrm{~mA} \mathrm{~h} \mathrm{~g}^{-1} \text { (first } \\
\text { cycle), } 300 \mathrm{~mA} \mathrm{~h} \\
\mathrm{~g}^{-1} \text { (cycle } 5 \text { ) }\end{array}$ & Not provided & S45 \\
\hline $\mathrm{Mg}_{1.03} \mathrm{Mn}_{0.97} \mathrm{SiO}_{4}$ & $\begin{array}{l}24{ }^{\circ} \mathrm{C}, \mathrm{Mg} \text { alloy } \\
\mathrm{AZ61}, 0.4 \mathrm{M}, 0.4 \\
\mathrm{M} \mathrm{APC} \text { electrolyte } \\
\text { (PhMgCl and } \mathrm{AlCl}_{3} \\
\text { in } 2: 1 \text { molar ratio) } \\
\text { in THF }\end{array}$ & Not provided & Not provided & $\begin{array}{l}1 \mathrm{~mA} \mathrm{~h} \mathrm{~g}^{-1} \text { (first } \\
\text { charge), } 4 \mathrm{~mA} \mathrm{~h} \mathrm{~g}{ }^{-1} \\
\text { (long-term cycling) }\end{array}$ & Not provided & S17 \\
\hline
\end{tabular}




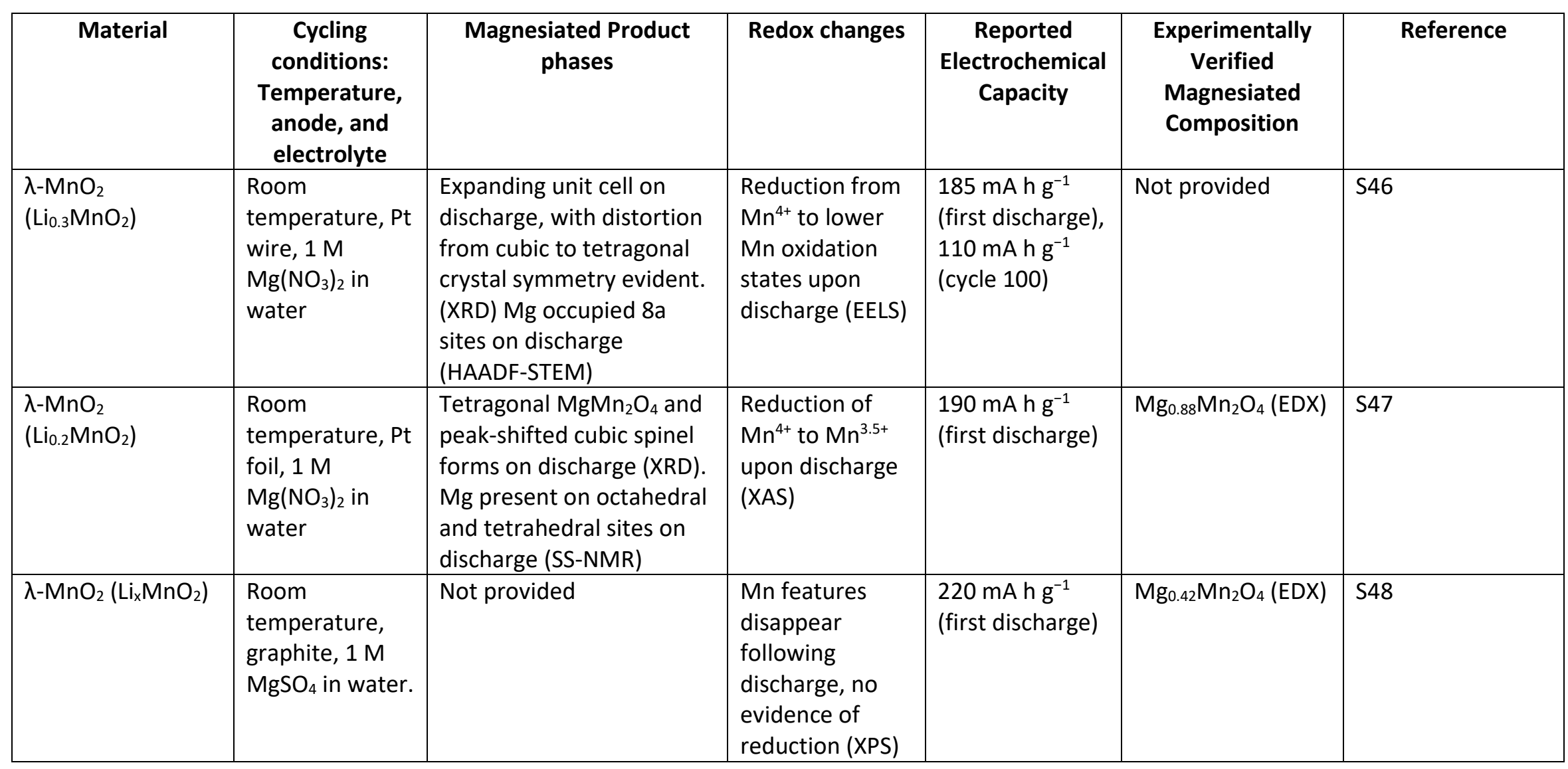




\begin{tabular}{|c|c|c|c|c|c|c|}
\hline Material & $\begin{array}{c}\text { Cycling } \\
\text { conditions: } \\
\text { Temperature, } \\
\text { anode, and } \\
\text { electrolyte } \\
\end{array}$ & $\begin{array}{l}\text { Magnesiated } \\
\text { Product phases }\end{array}$ & Redox changes & $\begin{array}{c}\text { Reported } \\
\text { Electrochemical } \\
\text { Capacity }\end{array}$ & $\begin{array}{l}\text { Experimentally } \\
\text { Verified } \\
\text { Magnesiated } \\
\text { Composition }\end{array}$ & Reference \\
\hline $\begin{array}{l}\mathrm{MgMn}_{2} \mathrm{O}_{4} \text { spinel } \\
\text { (tetragonal phase) }\end{array}$ & $\begin{array}{l}\text { Room } \\
\text { temperature, } \\
\text { capacitive carbon, } \\
0.5 \mathrm{M} \mathrm{Mg}\left(\mathrm{ClO}_{4}\right)_{2} \text { in } \\
\text { acetonitrile }\end{array}$ & $\begin{array}{l}\text { Formation of } \\
\mathrm{MgMnO}_{2} \text { rock salt } \\
\text { type phase on } \\
\mathrm{MgMn}_{2} \mathrm{O}_{4} \text { particle } \\
\text { surfaces ( } 2 \mathrm{~nm} \\
\text { thick) (HAADF- } \\
\text { STEM) }\end{array}$ & Not provided & $\begin{array}{l}100 \mathrm{~mA} \mathrm{~h} \mathrm{~g}^{-1} \text { (first } \\
\text { cycle), } 170 \mathrm{~mA} \mathrm{~h} \\
\mathrm{~g}^{-1} \text { (cycle } 27 \text { ) }\end{array}$ & Not provided & S49 \\
\hline $\begin{array}{l}\mathrm{MgMn}_{2} \mathrm{O}_{4} \text { spinel } \\
\text { (tetragonal phase) }\end{array}$ & $\begin{array}{l}\text { Room } \\
\text { temperature, a) Pt } \\
\text { foil, } 3 \mathrm{M} \mathrm{Mg}\left(\mathrm{NO}_{3}\right)_{2} \\
\text { in water b) } \mathrm{V}_{2} \mathrm{O}_{5} \\
0.5 \mathrm{M} \mathrm{Mg}\left(\mathrm{ClO}_{4}\right)_{2} \\
\text { EC:DEC }\end{array}$ & $\begin{array}{l}\text { a) } \lambda-\mathrm{MnO}_{2} \text { was } \\
\text { formed under a } \\
\text { constant-current } \\
\text { charge regime (but } \\
\text { not in CV } \\
\text { experiments) } \\
\text { (XRD) } \\
\text { b) The tetragonal } \\
\text { unit cell is } \\
\text { preserved on } \\
\text { charging, but } \\
\text { contracted along } \\
\text { the } c \text {-axis (XRD) }\end{array}$ & Not provided & $\begin{array}{l}\text { a) } 160 \mathrm{~mA} \mathrm{~h} \mathrm{~g}{ }^{-1} \\
\text { (first charge), } 85 \\
\mathrm{~mA} \mathrm{~h} \mathrm{~g}{ }^{-1} \text { (cycle 20) } \\
\text { b) } 190 \mathrm{~mA} \mathrm{~h} \mathrm{~g}{ }^{-1} \\
\text { (first cycle), } 120 \\
\mathrm{~mA} \mathrm{~h} \mathrm{~g}{ }^{-1} \text { (cycle 24) }\end{array}$ & Not provided & S50 \\
\hline $\begin{array}{l}\mathrm{MgMn}_{2} \mathrm{O}_{4} \text { spinel } \\
\text { (cubic phase) }\end{array}$ & $\begin{array}{l}\text { Room } \\
\text { temperature, } \\
\text { capacitive carbon, } \\
0.5 \mathrm{M} \mathrm{Mg}\left(\mathrm{ClO}_{4}\right)_{2} \text { in } \\
\text { acetonitrile }\end{array}$ & $\begin{array}{l}\text { Distortion of cubic } \\
\text { spinel to } \\
\text { tetragonal with } \\
\text { Mg extraction } \\
\text { (HAADF-STEM). }\end{array}$ & $\begin{array}{l}\text { Oxidation of } \mathrm{Mn}^{3+} \\
\text { evident with } \\
\text { charging, possibly } \\
\text { explained by } \mathrm{Mn}^{5+} \\
\text { formation (EELS) }\end{array}$ & $\begin{array}{l}120 \mathrm{~mA} \mathrm{~h}^{-1} \text { (first } \\
\text { charge), } 160 \mathrm{~mA} \mathrm{~h} \\
\mathrm{~g}^{-1} \text { (first discharge) }\end{array}$ & $\begin{array}{l}\mathrm{Mg}_{0.62} \mathrm{Mn}_{2} \mathrm{O}_{4} \text { on } \\
\text { charge } \\
\text { (ABF/HAADF/EELS } \\
\text { STEM) }\end{array}$ & S51 \\
\hline $\mathrm{MgMn}_{2} \mathrm{O}_{4} / \mathrm{V}_{2} \mathrm{O}_{5}$ & $\begin{array}{l}100{ }^{\circ} \mathrm{C}, \mathrm{Mg} \text { foil, } 0.3 \\
\mathrm{M}[\mathrm{Mg}(\mathrm{G} 4)][\mathrm{TFSA}]_{2} \\
\text { in [P13][TFSA] }\end{array}$ & Not provided & Not provided & $\begin{array}{l}140 \mathrm{~mA} \mathrm{~h} \mathrm{~g}^{-1} \text { (first } \\
\text { discharge), } 45 \mathrm{~mA} \\
\mathrm{~h} \mathrm{~g}^{-1} \text { (cycle } 100 \text { ) }\end{array}$ & Not provided & S52 \\
\hline
\end{tabular}




\begin{tabular}{|c|c|c|c|c|c|c|}
\hline Material & $\begin{array}{c}\text { Cycling } \\
\text { conditions: } \\
\text { Temperature, } \\
\text { anode, and } \\
\text { electrolyte } \\
\end{array}$ & $\begin{array}{l}\text { Magnesiated } \\
\text { Product phases }\end{array}$ & Redox changes & $\begin{array}{c}\text { Reported } \\
\text { Electrochemical } \\
\text { Capacity }\end{array}$ & $\begin{array}{l}\text { Experimentally } \\
\text { Verified } \\
\text { Magnesiated } \\
\text { Composition }\end{array}$ & Reference \\
\hline $\begin{array}{l}\mathrm{MgMn}_{2} \mathrm{O}_{4} \text { spinel } \\
\text { (cubic phase thin } \\
\text { film) }\end{array}$ & $\begin{array}{l}\text { Room } \\
\text { temperature, } \\
\text { capacitive carbon, } \\
0.2 \mathrm{M} \mathrm{Mg}(\mathrm{TFSI})_{2} \text { in } \\
\mathrm{PC}\end{array}$ & $\begin{array}{l}\text { Unit cell } \\
\text { contraction with } \\
\text { charge, expansion } \\
\text { with discharge } \\
\text { (Specular XRD) }\end{array}$ & $\begin{array}{l}\text { Partial oxidation of } \\
\mathrm{Mn}^{3+} \text { to } \mathrm{Mn}^{4+} \text { on } \\
\text { charge, reduction } \\
\text { to } \mathrm{Mn}^{3+} \text { and } \mathrm{Mn}^{2+} \\
\text { on discharge } \\
\text { (XANES and XPS) }\end{array}$ & $\begin{array}{l}250 \mathrm{~mA} \mathrm{~h} \mathrm{~g}^{-1} \\
\text { (second charge), } \\
70 \mathrm{~mA} \mathrm{~h} \mathrm{~g}^{-1} \text { (cycle } \\
\text { 38) }\end{array}$ & $\begin{array}{l}\mathrm{Mg}_{0.67} \mathrm{Mn}_{2} \mathrm{O}_{4} \\
\text { (charged), } \\
\mathrm{MgMn}_{2} \mathrm{O}_{4} \\
\text { (discharged) (XPS) }\end{array}$ & S53 \\
\hline $\begin{array}{l}\mathrm{Mg}_{0.5} \mathrm{Mn}_{2.5} \mathrm{O}_{4} \\
\text { spinel (tetragonal } \\
\text { phase) }\end{array}$ & $\begin{array}{l}\text { Room } \\
\text { temperature, } \mathrm{Pt} \\
\text { foil, } 1 \mathrm{M} \mathrm{Mg}\left(\mathrm{NO}_{3}\right)_{2} \\
\text { in water }\end{array}$ & $\begin{array}{l}\text { Transition from } \\
\text { spinel } \rightarrow \text { layered } \rightarrow \\
\text { spinel structures } \\
\text { during pristine } \rightarrow \\
\text { charged } \rightarrow \\
\text { discharged } \\
\text { transitions (XRD) }\end{array}$ & $\begin{array}{l}\text { Oxidation of } \\
\mathrm{Mn}^{2.67+} \text { to } \mathrm{Mn}^{4+} \text { on } \\
\text { charge, reduction } \\
\text { to } \mathrm{Mn}^{3+} \text { on } \\
\text { discharge (XAS) }\end{array}$ & $\begin{array}{l}560 \mathrm{~mA} \mathrm{~h} \mathrm{~g}^{-1} \text { (first } \\
\text { charge and } \\
\text { discharge) }\end{array}$ & $\begin{array}{l}\mathrm{Mg} / \mathrm{Mn} \text { ratio of } \\
0.5 \text { upon discharge } \\
(0.167 \text { in the } \\
\text { pristine) from } \\
\text { Rietveld analysis }\end{array}$ & S54 \\
\hline $\begin{array}{l}\mathrm{MgMn}_{2} \mathrm{O}_{4} \text { spinel } \\
\text { (tetragonal phase) }\end{array}$ & $\begin{array}{l}25^{\circ} \mathrm{C} \text {, capacitive } \\
\text { carbon, } 0.5 \mathrm{M} \\
\mathrm{Mg}\left(\mathrm{ClO}_{4}\right)_{2} \text { in } \\
\text { acetonitrile }\end{array}$ & Not provided & Not provided & $\begin{array}{l}65 \mathrm{~mA} \mathrm{~h} \mathrm{~g}^{-1} \text { (first } \\
\text { charge), } 40 \mathrm{~mA} \mathrm{~h} \\
\mathrm{~g}^{-1} \text { (cycle } 30 \text { ) }\end{array}$ & Not provided & S56 \\
\hline $\begin{array}{l}\mathrm{MgMn}_{2} \mathrm{O}_{4} \text { spinel } \\
\text { with vanadate } \\
\text { coating }\end{array}$ & $\begin{array}{l}100{ }^{\circ} \mathrm{C}, \mathrm{Mg} \text { foil, } 0.3 \\
\mathrm{M}[\mathrm{Mg}(\mathrm{G} 4)][\mathrm{TFSA}]_{2} \\
\text { in [P13][TFSA] }\end{array}$ & $\begin{array}{l}\text { No changes in } \\
\text { crystal structure } \\
\text { on discharge } \\
\text { (SAED) }\end{array}$ & Not provided & $\begin{array}{l}170 \mathrm{~mA} \mathrm{~h} \mathrm{~g}^{-1} \text { (first } \\
\text { discharge), } 35 \mathrm{~mA} \\
\mathrm{~h} \mathrm{~g}^{-1} \text { (cycle } 47 \text { ) }\end{array}$ & $\mathrm{Mg}_{1.5} \mathrm{Mn}_{2} \mathrm{O}_{4}(\mathrm{EDX})$ & S57 \\
\hline
\end{tabular}


Manganese Oxides

\begin{tabular}{|c|c|c|c|c|c|c|}
\hline Material & $\begin{array}{c}\text { Cycling } \\
\text { conditions: } \\
\text { Temperature, } \\
\text { anode, and } \\
\text { electrolyte } \\
\end{array}$ & $\begin{array}{l}\text { Magnesiated } \\
\text { Product phases }\end{array}$ & Redox changes & $\begin{array}{c}\text { Reported } \\
\text { Electrochemical } \\
\text { Capacity }\end{array}$ & $\begin{array}{l}\text { Experimentally } \\
\text { Verified } \\
\text { Magnesiated } \\
\text { Composition }\end{array}$ & Reference \\
\hline $\begin{array}{l}\mathrm{MgMn}_{1.8} \mathrm{Sr}_{0.2} \mathrm{O}_{4} \\
\text { spinel }\end{array}$ & $\begin{array}{l}\text { Room } \\
\text { temperature, } \mathrm{Mg} \\
\text { foil, } 1 \mathrm{M} \\
\left(\mathrm{Mg}\left(\mathrm{CF}_{3} \mathrm{SO}_{3}\right)_{2} \text { in }\right. \\
1: 1 \mathrm{EC} / \mathrm{DME}^{2}\end{array}$ & Not provided & Not provided & $\begin{array}{l}160 \mathrm{~mA} \mathrm{~h} \mathrm{~g}^{-1} \text { (first } \\
\text { discharge), } 60 \mathrm{~mA} \\
\mathrm{~h} \mathrm{~g}^{-1} \text { (cycle } 10 \text { ) }\end{array}$ & Not provided & S58 \\
\hline $\begin{array}{l}\mathrm{MgMn}_{2} \mathrm{O}_{4} \text { spinel } \\
\text { (tetragonal phase) }\end{array}$ & $\begin{array}{l}150{ }^{\circ} \mathrm{C}, \mathrm{Mg} \text { foil, } 10 \\
\mathrm{~mol} \% \mathrm{Mg}(\mathrm{TFSA})_{2} \text { in } \\
90 \text { mol\% CsTFSA }\end{array}$ & $\begin{array}{l}\text { Reversible two- } \\
\text { phase reaction of } \\
\text { spinel } \mathrm{Mg}_{1-x} \mathrm{Mn}_{2} \mathrm{O}_{4} \\
\text { and rock-salt type } \\
\mathrm{Mg}_{1-\mathrm{x}} \mathrm{MnO}_{2} \text { on } \mathrm{Mg} \\
\text { removal/insertion } \\
\text { (XRD) }\end{array}$ & $\begin{array}{l}\text { Partial Oxidation } \\
\text { of } \mathrm{Mn}^{3+} \text { to } \mathrm{Mn}^{4+} \\
\text { upon charge (XPS) }\end{array}$ & $\begin{array}{l}138 \mathrm{~mA} \mathrm{~h}^{-1} \text { (first } \\
\text { charge), } 185 \mathrm{~mA} \mathrm{~h} \\
\mathrm{~g}^{-1} \text { (first discharge) }\end{array}$ & $\begin{array}{l}\mathrm{Mg}_{0.58} \mathrm{Mn}_{2} \mathrm{O}_{4} \text { in the } \\
\text { charged state (ICP- } \\
\mathrm{AES} \text { ). Confirmation } \\
\text { of } \mathrm{Mg} \text { vacancies } \\
\text { (STEM). }\end{array}$ & S59 \\
\hline
\end{tabular}


Manganese Oxides

\begin{tabular}{|c|c|c|c|c|c|c|}
\hline Material & $\begin{array}{c}\text { Cycling } \\
\text { conditions: } \\
\text { Temperature, } \\
\text { anode, and } \\
\text { electrolyte }\end{array}$ & $\begin{array}{l}\text { Magnesiated } \\
\text { Product phases }\end{array}$ & Redox changes & $\begin{array}{c}\text { Reported } \\
\text { Electrochemical } \\
\text { Capacity }\end{array}$ & $\begin{array}{l}\text { Experimentally } \\
\text { Verified } \\
\text { Magnesiated } \\
\text { Composition }\end{array}$ & Reference \\
\hline $\begin{array}{l}\mathrm{MgMn}_{2} \mathrm{O}_{4} \cdot 0.7 \mathrm{H}_{2} \mathrm{O} \\
\text { spinel (tetragonal } \\
\text { phase) }\end{array}$ & $\begin{array}{l}\text { Room } \\
\text { temperature, } \\
\text { capacitive carbon, } \\
0.5 \mathrm{M} \mathrm{Mg}(\mathrm{TFSI})_{2} \\
\text { and } 0.5 \mathrm{M} \text { dipro- } \\
\text { glyme in } \\
\text { acetonitrile with a) } \\
\text { no water added or } \\
\text { b) } 3 \mathrm{M} \text { water } \\
\text { added }\end{array}$ & $\begin{array}{l}\text { a) New phase } \\
\text { formation with } \\
\text { charge (no } \\
\text { information on } \\
\text { new phase given) } \\
\text { b) No obvious } \\
\text { crystallographic } \\
\text { charges with } \\
\text { charge and } \\
\text { discharge (XRD) }\end{array}$ & Not provided & 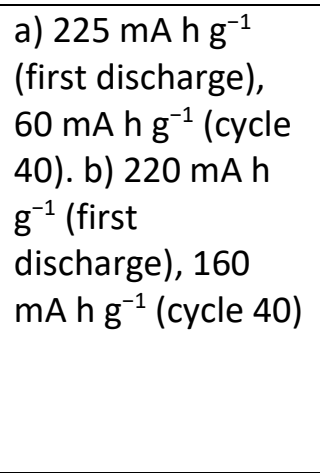 & Not provided & $S 60$ \\
\hline $\begin{array}{l}\mathrm{Mg}\left(\mathrm{Mn}_{0.4} \mathrm{Fe}_{0.6}\right)_{2} \mathrm{O}_{4} \\
\text { (cubic phase) }\end{array}$ & $\begin{array}{l}100^{\circ} \mathrm{C}, \mathrm{Mg} \text { foil, } \\
0.01 \mathrm{M} \mathrm{AgNO}_{3} \text { and } \\
0.10 \mathrm{M} \mathrm{Mg}\left(\mathrm{TFSA}_{2}\right. \\
\text { in triglyme }\end{array}$ & $\begin{array}{l}\text { Formation of rock- } \\
\text { salt phase } \\
\text { observed on } \\
\text { discharge, } \\
\text { reformation of } \\
\text { spinel on charge. } \\
\text { (XRD) }\end{array}$ & $\begin{array}{l}\text { Partially reversible } \\
\mathrm{Mn}^{2+} / \mathrm{Mn}^{3+} \text { redox } \\
\text { (major } \\
\text { contribution), fully } \\
\text { reversible } \\
\mathrm{Fe}^{2+} / \mathrm{Fe}^{3+} \text { redox } \\
\text { (minor } \\
\text { contribution). } \\
\text { (XAS) }\end{array}$ & $\begin{array}{l}180 \mathrm{~mA} \mathrm{~h} \mathrm{~g}^{-1} \text { (first } \\
\text { discharge), } 50 \mathrm{~mA} \\
\mathrm{~h} \mathrm{~g}^{-1} \text { (cycle } 30 \text { ) }\end{array}$ & Not provided & S61 \\
\hline $\mathrm{MgNiMnO}_{4}$ & $\begin{array}{l}80^{\circ} \mathrm{C}, \mathrm{Mo}_{6} \mathrm{~S}_{8}, 0.25 \\
\mathrm{M} \mathrm{Mg}(\mathrm{TFSI})_{2} \text { in } \\
\mathrm{BMPTFSI}\end{array}$ & $\begin{array}{l}\text { Dissolution of } \\
\mathrm{MgNiMnO}_{4} \text { and } \\
\text { deposition on } \\
\text { anode (HRSEM } \\
\text { and EDS) }\end{array}$ & Not provided & Not provided & $\begin{array}{l}\text { Dissolution of } \\
\mathrm{MgNiMnO}_{4} \text { and } \\
\text { deposition on } \\
\text { anode (HRSEM } \\
\text { and EDS) }\end{array}$ & S62 \\
\hline
\end{tabular}


Manganese Oxides

\begin{tabular}{|c|c|c|c|c|c|c|}
\hline Material & $\begin{array}{c}\text { Cycling } \\
\text { conditions: } \\
\text { Temperature, } \\
\text { anode, and } \\
\text { electrolyte }\end{array}$ & $\begin{array}{l}\text { Magnesiated } \\
\text { Product phases }\end{array}$ & Redox changes & $\begin{array}{c}\text { Reported } \\
\text { Electrochemical } \\
\text { Capacity }\end{array}$ & $\begin{array}{l}\text { Experimentally } \\
\text { Verified } \\
\text { Magnesiated } \\
\text { Composition }\end{array}$ & Reference \\
\hline $\mathrm{Mn}_{3} \mathrm{O}_{4}$ & $\begin{array}{l}\text { Room } \\
\text { temperature, } \mathrm{Mg} \\
\text { foil, } 0.4 \mathrm{M} \mathrm{APC} \\
\text { electrolyte } \\
\text { (PhMgCl and } \mathrm{AlCl}_{3} \\
\text { in } 2: 1 \text { molar ratio) } \\
\text { in } \mathrm{THF}\end{array}$ & $\begin{array}{l}\text { Broadened peaks } \\
\text { upon discharge, } \\
\text { with no shift in } \\
\text { Bragg reflections } \\
\text { or new phases, } \\
\text { recovery of } \\
\text { intensity on } \\
\text { charge(XRD) }\end{array}$ & Not provided & $\begin{array}{l}190 \mathrm{~mA} \mathrm{~h} \mathrm{~g}^{-1} \text { (first } \\
\text { cycle) } \\
90 \mathrm{~mA} \mathrm{~h} \mathrm{~g}^{-1} \text { (cycle } \\
20 \text { ) }\end{array}$ & Not provided & S63 \\
\hline $\mathrm{Mn}_{3} \mathrm{O}_{4}$ & $\begin{array}{l}\text { Room } \\
\text { temperature, } \mathrm{Mg} \\
\text { foil, } 0.4 \mathrm{M} \mathrm{APC} \\
\text { electrolyte } \\
\text { ( } \mathrm{PhMgCl} \text { and } \mathrm{AlCl}_{3} \\
\text { in } 2: 1 \text { molar ratio) } \\
\text { in THF, tetraglyme, } \\
\text { or DME. }\end{array}$ & $\begin{array}{l}\text { Rock-salt type } \\
\text { (Mg,Mn)O formed } \\
\text { at } \mathrm{Mn}_{3} \mathrm{O}_{4} \text { particle } \\
\text { surfaces, no bulk } \\
\text { reaction on } \\
\text { discharge(HAADF- } \\
\text { STEM) }\end{array}$ & $\begin{array}{l}\text { Mn oxidation state } \\
\text { unchanged in the } \\
\text { bulk, reduction of } \\
\mathrm{Mn}^{3+} \text { to } \mathrm{Mn}^{2+} \text { at } \\
\text { the surface on } \\
\text { discharge (EELS) }\end{array}$ & $\begin{array}{l}800 \mathrm{~mA} \mathrm{~h} \mathrm{~g}^{-1} \text { (first } \\
\text { cycle), } 580 \mathrm{~mA} \mathrm{~h} \\
\mathrm{~g}^{-1} \text { (cycle } 5 \text { ) }\end{array}$ & Not provided & S64 \\
\hline $\mathrm{Mn}_{2.15} \mathrm{Co}{ }_{0.37} \mathrm{O}_{4}$ & $\begin{array}{l}\text { Room } \\
\text { temperature, } \mathrm{Mg} \\
\text { rod, } 0.1 \mathrm{M} \\
\mathrm{Mg}\left(\mathrm{ClO}_{4}\right)_{2} \text { in } \mathrm{PC}\end{array}$ & Not provided & Not provided & $\begin{array}{l}57 \mathrm{~mA} \mathrm{~h} \mathrm{~g}^{-1} \text { (first } \\
\text { cycle), } 25 \mathrm{~mA} \mathrm{~h} \mathrm{~g}^{-1} \\
\text { (cycle } 30 \text { ) }\end{array}$ & Not provided & S65 \\
\hline
\end{tabular}


Manganese Oxides

\begin{tabular}{|c|c|c|c|c|c|c|}
\hline Material & $\begin{array}{c}\text { Cycling } \\
\text { conditions: } \\
\text { Temperature, } \\
\text { anode, and } \\
\text { electrolyte }\end{array}$ & $\begin{array}{l}\text { Magnesiated } \\
\text { Product phases }\end{array}$ & Redox changes & $\begin{array}{l}\text { Reported } \\
\text { Electrochemi } \\
\text { cal Capacity }\end{array}$ & $\begin{array}{c}\text { Experimentally } \\
\text { Verified Magnesiated } \\
\text { Composition }\end{array}$ & Reference \\
\hline $\mathrm{Mn}_{3} \mathrm{O}_{4}$ & $\begin{array}{l}\text { Room } \\
\text { temperature, } \\
\mathrm{Mn}_{3} \mathrm{O}_{4}, 1 \mathrm{M} \mathrm{MgSO}_{4} \\
\text { in water }\end{array}$ & $\begin{array}{l}\text { Formation of } \\
\text { Birnessite }(\delta- \\
\mathrm{MnO}_{2} \cdot \mathrm{xH}_{2} \mathrm{O} \text { ) after } \\
4 \text { cycles (XRD). } \\
\text { Layer-by-layer } \\
\text { formation of } \\
\text { Birnessite from } \\
\mathrm{Mn}_{3} \mathrm{O}_{4} \text { during } \\
\text { discharge with } \\
\text { peeling off (ABF- } \\
\text { STEM) Insertion of } \\
\text { crystal water with } \\
\text { cycling (TGA) and } \\
\mathrm{H}_{3} \mathrm{O}^{+} \text {species found } \\
\text { at the surface ( } 1 \\
\mathrm{~nm} \text { deep) (TOF- } \\
\text { SIMS) }\end{array}$ & Not provided & $\begin{array}{l}90 \mathrm{~mA} \mathrm{~h} \mathrm{~g}^{-1} \\
\text { (first } \\
\text { discharge), } \\
220 \mathrm{~mA} \mathrm{~h} \mathrm{~g}{ }^{-1} \\
\text { (cycle 4) }\end{array}$ & $\begin{array}{l}\mathrm{Mg}_{0.36} \mathrm{Mn}_{2} \mathrm{O}_{4} \\
\text { (discharged) }\end{array}$ & $S 66,67$ \\
\hline $\mathrm{Mn}_{2} \mathrm{O}_{3}$ & $\begin{array}{l}\text { Room } \\
\text { temperature, a) Pt } \\
\text { wire, } 0.1 \mathrm{M} \mathrm{MgCl} \\
\text { in water b) } \mathrm{Mg} \\
\text { wire, } 0.5 \mathrm{M} \\
\mathrm{Mg}\left(\mathrm{ClO}_{4}\right)_{2} \text { in } \\
\text { acetonitrile }\end{array}$ & $\begin{array}{l}\text { a) Dissolution to } \\
\text { form } \mathrm{Mn}^{2+} \text { on } \\
\text { discharge (UV-vis } \\
\text { spectroscopy) }\end{array}$ & Not provided & $\begin{array}{l}\text { a) } 2.8 \mathrm{~mA} \mathrm{~h} \\
\mathrm{~g}^{-1} \text { (unknown } \\
\text { cycle), b) Not } \\
\text { provided }\end{array}$ & $\begin{array}{l}\text { a) Mg found } \\
\text { predominantly at the } \\
\text { surface when } \\
\text { discharged, mostly } \\
\text { extracted on charge. b) } \\
\text { Mg found at the } \\
\text { surface and in bulk } \\
\text { when discharged, only } \\
\text { partially removed on } \\
\text { charge (XPS depth } \\
\text { profiling) }\end{array}$ & S68 \\
\hline
\end{tabular}




\begin{tabular}{|c|c|c|c|c|c|c|}
\hline Material & $\begin{array}{l}\text { Cycling } \\
\text { conditions: } \\
\text { Temperature, } \\
\text { anode, and } \\
\text { electrolyte }\end{array}$ & $\begin{array}{l}\text { Magnesiated } \\
\text { Product phases }\end{array}$ & Redox changes & $\begin{array}{l}\text { Reported } \\
\text { Electrochemi } \\
\text { cal Capacity }\end{array}$ & $\begin{array}{c}\text { Experimentally } \\
\text { Verified Magnesiated } \\
\text { Composition }\end{array}$ & Reference \\
\hline $\begin{array}{l}\text { a) } \\
\mathrm{Ag}_{1.22} \mathrm{Mn}_{8} \mathrm{O}_{15.6} \cdot 2.3 \\
\left.\mathrm{H}_{2} \mathrm{O}, \mathrm{b}\right) \\
\mathrm{K}_{0.81} \mathrm{Mn}_{8} \mathrm{O}_{14.5} \cdot 2.7 \mathrm{H}_{2} \\
\mathrm{O}\end{array}$ & $\begin{array}{l}\text { Room } \\
\text { temperature, } \\
\text { carbon, } 0.5 \mathrm{M} \\
\mathrm{Mg}(\mathrm{TFSI})_{2} \text { in } \\
\text { acetonitrile with } 3 \\
\mathrm{M} \text { water added }\end{array}$ & $\begin{array}{l}\text { a) and b) } \\
\text { Amorphization } \\
\text { following cycling } \\
\text { (XRD, SAED)) }\end{array}$ & $\begin{array}{l}\text { a) and b) Some } \\
\text { evidence of } \\
\text { reduction of } \mathrm{Mn} \\
\text { following } \\
\text { extended cycling. } \\
\text { (XPS) }\end{array}$ & $\begin{array}{l}\text { a) } 180 \mathrm{~mA} \mathrm{~h} \\
\mathrm{~g}^{-1} \text { (first } \\
\text { discharge), } 90 \\
\mathrm{~mA} \mathrm{~h} \mathrm{~g}^{-1} \\
\text { (cycle } 20 \text { ) b) } \\
250 \mathrm{~mA} \mathrm{~h} \mathrm{~g} \mathrm{~g}^{-1} \\
\text { (first } \\
\text { discharge), } \\
180 \mathrm{~mA} \mathrm{~h} \mathrm{~g} \\
\text { (cycle 20) }\end{array}$ & Not provided & S69 \\
\hline $\begin{array}{l}\mathrm{Na}_{0.44} \mathrm{MnO}_{2}(2 \times 4 \\
\text { tunnel structured } \\
\left.\mathrm{MnO}_{2}\right)\end{array}$ & $\begin{array}{l}\text { Room } \\
\text { temperature, } \\
\text { carbon, } 0.4 \mathrm{M} \\
\mathrm{Mg}(\mathrm{TFSI})_{2} \text { with } 2.4 \\
\mathrm{M} \text { water and } 0.5 \\
\mathrm{M} \text { dipro-glyme in } \\
\text { acetonitrile }\end{array}$ & $\begin{array}{l}\text { Evidence of phase } \\
\text { change/lattice } \\
\text { changes after } 100 \\
\text { cycles (XRD) }\end{array}$ & Not provided & $\begin{array}{l}310 \mathrm{~mA} \mathrm{~h} \mathrm{~g}^{-1} \\
\text { (first } \\
\text { discharge), } 50 \\
\mathrm{~mA} \mathrm{~h} \mathrm{~g}^{-1} \\
\text { (cycle 100) }\end{array}$ & Not provided & S70 \\
\hline $\mathrm{MnO}_{2} / \mathrm{V}_{2} \mathrm{C}$ & $\begin{array}{l}\text { Room } \\
\text { temperature, Mg } \\
\text { foil, APC } \\
\text { electrolyte } \\
\text { (concentration not } \\
\text { given) }\end{array}$ & Not provided & Not provided & $\begin{array}{l}190 \mathrm{~mA} \mathrm{~h} \mathrm{~g}^{-1} \\
\text { (first cycle), } \\
170 \mathrm{~mA} \mathrm{~h} \mathrm{~g}^{-1} \\
\text { (cycle 5) }\end{array}$ & Not provided & S71 \\
\hline
\end{tabular}


Molybdenum oxides

\begin{tabular}{|c|c|c|c|c|c|c|}
\hline Material & $\begin{array}{l}\text { Cycling conditions: } \\
\text { Temperature, anode, } \\
\text { and electrolyte }\end{array}$ & $\begin{array}{l}\text { Magnesiated } \\
\text { Product phases }\end{array}$ & Redox changes & $\begin{array}{c}\text { Reported } \\
\text { Electrochemical } \\
\text { Capacity }\end{array}$ & $\begin{array}{l}\text { Experimentally } \\
\text { Verified } \\
\text { Magnesiated } \\
\text { Composition }\end{array}$ & Reference \\
\hline $\mathrm{MoO}_{3}$ & $\begin{array}{l}80{ }^{\circ} \mathrm{C}, \mathrm{Mg} \text { foil, a) } \\
\text { 3:41:56 wt\% } \\
\mathrm{MgCl}_{2}: \mathrm{EMIC}^{\mathrm{AlCl}} \mathrm{Al}_{3} \text { b) } 1 \\
\mathrm{M} \mathrm{Mg}\left(\mathrm{ClO}_{4}\right)_{2} \text { in } \\
\text { acetonitrile with } 3 \\
\text { mol\% water }\end{array}$ & Not provided & Not provided & 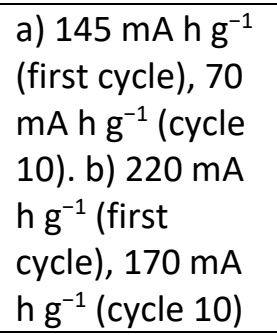 & Not provided & S72 \\
\hline $\mathrm{MoO}_{3}$ & $\begin{array}{l}\text { Room temperature, } \\
\mathrm{Pt} \text { foil, } 0.1 \mathrm{M} \\
\mathrm{Mg}\left(\mathrm{ClO}_{4}\right)_{2} \text { in PC }\end{array}$ & $\begin{array}{l}\text { Partially } \\
\text { reversible } \\
\text { expansion and } \\
\text { contraction of } \\
\text { lattice } \\
\text { parameters with } \\
\text { discharge and } \\
\text { charge } \\
\text { respectively, } \\
\text { especially the } \\
\text { layer } b \text {-axis } \\
\text { (XRD) }\end{array}$ & $\begin{array}{l}\text { Reduction of } \\
\mathrm{Mo}^{6+} \text { to a } \\
\text { mixture of } \\
\mathrm{Mo}^{3+}, \mathrm{Mo}^{4+} \\
\mathrm{Mo}^{5+}, \mathrm{Mo}^{6+} \text { on } \\
\text { discharge } \\
\text { (photoemission } \\
\text { spectroscopy). } \\
\text { Evidence of } \\
\text { surface } \\
\text { reduction on } \\
\text { discharge (XPS) }\end{array}$ & Not reported & Not provided & $S 73,74$ \\
\hline
\end{tabular}




\begin{tabular}{|c|c|c|c|c|c|c|}
\hline Material & $\begin{array}{l}\text { Cycling conditions: } \\
\text { Temperature, anode, } \\
\text { and electrolyte }\end{array}$ & $\begin{array}{l}\text { Magnesiated } \\
\text { Product phases }\end{array}$ & Redox changes & $\begin{array}{c}\text { Reported } \\
\text { Electrochemical } \\
\text { Capacity }\end{array}$ & $\begin{array}{l}\text { Experimentally } \\
\text { Verified } \\
\text { Magnesiated } \\
\text { Composition }\end{array}$ & Reference \\
\hline $\mathrm{MoO}_{3}$ (thin film) & $\begin{array}{l}\text { Room temperature, } \\
\text { capacitive carbon, } \\
0.1 \mathrm{M} \mathrm{MgTFSI}_{2} \text { in } \\
\text { acetonitrile }\end{array}$ & $\begin{array}{l}\text { Expansion of } \\
\text { layer axis ( } b \text { - } \\
\text { axis) on } \\
\text { discharge with } \\
\text { additional peaks } \\
\text { forming, } \\
\text { contraction on } \\
\text { charge. (XRD) } \\
\text { Change in local } \\
\text { structure on } \\
\text { discharge } \\
\text { (Raman). }\end{array}$ & Not provided & $\begin{array}{l}230 \mathrm{~mA} \mathrm{~h} \mathrm{~g}^{-1} \\
\text { (first cycle) }\end{array}$ & Not provided & S75 \\
\hline a) $\mathrm{MoO}_{3}$, b) $\mathrm{MoO}_{2.8} \mathrm{~F}_{0.2}$ & $\begin{array}{l}\text { Room temperature, } \\
\text { capacitive carbon, } 0.2 \\
\mathrm{M} \mathrm{Mg}(\mathrm{TFSI})_{2} \text { in } \mathrm{PC}\end{array}$ & $\begin{array}{l}\text { a) Not provided, } \\
\text { b) Two-phase } \\
\text { insertion } \\
\text { mechanism, } \\
\text { insertion breaks } \\
\text { crystal } \\
\text { symmetry. } \\
\text { Recharging did } \\
\text { not yield the } \\
\text { original crystal } \\
\text { structure (XRD) } \\
\text { Disordered Mg } \\
\text { insertion in the } \\
\text { oxide lattice on } \\
\text { discharge ( }{ }^{25} \mathrm{Mg} \\
\mathrm{NMR} \text { ) }\end{array}$ & Not provided & 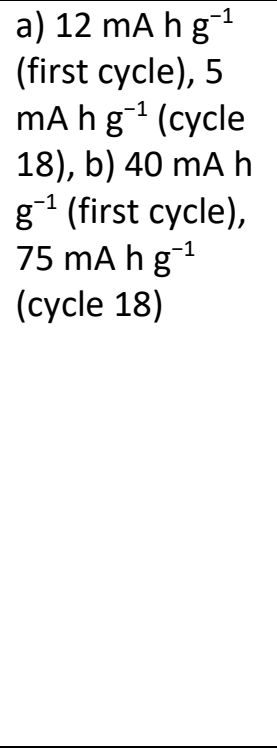 & $\begin{array}{l}\text { a) Not } \\
\text { provided, b) } \\
\mathrm{Mg}_{0.09} \mathrm{MoO}_{2.8} \mathrm{~F}_{0.2} \\
\text { (Rietveld } \\
\text { refinement) No } \\
\text { protons or } \\
\text { solvent species } \\
\text { detected }\left({ }^{1} \mathrm{H}\right. \\
\text { NMR) }\end{array}$ & S76 \\
\hline
\end{tabular}




\begin{tabular}{|c|c|c|c|c|c|c|}
\hline Material & $\begin{array}{l}\text { Cycling conditions: } \\
\text { Temperature, anode, } \\
\text { and electrolyte }\end{array}$ & $\begin{array}{l}\text { Magnesiated } \\
\text { Product phases }\end{array}$ & Redox changes & $\begin{array}{l}\text { Reported } \\
\text { Electrochemical } \\
\text { Capacity }\end{array}$ & $\begin{array}{l}\text { Experimentally } \\
\text { Verified } \\
\text { Magnesiated } \\
\text { Composition }\end{array}$ & Reference \\
\hline $\begin{array}{l}\mathrm{Na}_{15}\left\{\left[\mathrm{Mo}_{154} \mathrm{O}_{462} \mathrm{H}_{14}\left(\mathrm{H}_{2} \mathrm{O}\right)_{70}\right]_{0.5}\right. \\
\left.\left[\mathrm{Mo}_{152} \mathrm{O}_{457} \mathrm{H}_{14}\left(\mathrm{H}_{2} \mathrm{O}\right)_{68}\right]_{0.5}\right\} \cdot c a \\
400 \mathrm{H}_{2} \mathrm{O}\end{array}$ & $\begin{array}{l}\text { Room temperature, } \\
\mathrm{Mg} \text { foil, } 0.25 \mathrm{M} \mathrm{APC} \\
\text { electrolyte ( } \mathrm{PhMgCl} \\
\text { and } \mathrm{AlCl}_{3} \text { in } 2: 1 \text { molar } \\
\text { ratio) in THF }\end{array}$ & Not provided & Not provided & $\begin{array}{l}275 \mathrm{~mA} \mathrm{~h} \mathrm{~g}^{-1} \\
\text { (first } \\
\text { discharge), } 55 \\
\mathrm{~mA} \mathrm{~h} \mathrm{~g}^{-1} \text { (cycle } \\
500 \text { ) }\end{array}$ & Not provided & S77 \\
\hline $\mathrm{Mg}_{2} \mathrm{Mo}_{3} \mathrm{O}_{8}$ & $\begin{array}{l}\text { Room temperature, } \\
\mathrm{Mg} \text { foil, a) } 0.4 \mathrm{M} \mathrm{APC} \\
\text { electrolyte ( } \mathrm{PhMgCl} \\
\text { and } \mathrm{AlCl} \mathrm{I}_{3} \text { in } 2: 1 \mathrm{molar} \\
\text { ratio) in } \mathrm{THF}, \mathrm{b}) 0.5 \mathrm{M} \\
\mathrm{Mg}\left(\mathrm{ClO}_{4}\right)_{2} \text { in water }\end{array}$ & $\begin{array}{l}\text { Crystal structure } \\
\text { changes little } \\
\text { with chemical } \\
\text { demagnesiation, } \\
\text { suggesting a } \\
\text { two-phase } \\
\text { reaction } \\
\text { generating a } \\
\text { demagnesiated } \\
\text { amorphous } \\
\text { phase. (XRD) }\end{array}$ & Not provided & $\begin{array}{l}\text { a) and b): }<1 \\
\mathrm{~mA} \mathrm{~h} \mathrm{~g}^{-1} \text { (first } \\
\text { cycle) }\end{array}$ & Not provided & S78 \\
\hline $\mathrm{Mg}_{2} \mathrm{Mo}_{3} \mathrm{O}_{8}$ & $\begin{array}{l}\text { a) } 90{ }^{\circ} \mathrm{C}, \text { AZ31, } 0.5 \mathrm{M} \\
{[\mathrm{Mg}(\mathrm{G} 4)]} \\
{[\mathrm{TFSA}]_{2} /\left[\mathrm{PYR}_{13}\right][\mathrm{TFSA}]} \\
\text { electrolyte. b) } 60^{\circ} \mathrm{C} \text {, } \\
\text { Li metal, } 1 \mathrm{M} \mathrm{LiPF} / \\
\text { EC:DMC (1:2 vol. } \\
\text { ratio) }\end{array}$ & $\begin{array}{l}\text { b) Small } \\
\text { contraction of } \\
\text { unit cell and } \\
\text { some loss of } \\
\text { crystallinity } \\
\text { observed upon } \\
\text { charge (XRD) }\end{array}$ & $\begin{array}{l}\text { b) Oxidation of } \\
\mathrm{Mo}^{4+} \text { to } \mathrm{Mo}^{4.3+} \\
\text { on charge } \\
\text { (XANES) }\end{array}$ & $\begin{array}{l}\text { a) } 18 \mathrm{~mA} \mathrm{~h} \mathrm{~g}^{-1} \\
\text { (first charge), } \\
90 \mathrm{~mA} \mathrm{~h} \mathrm{~g}^{-1} \\
\text { (first } \\
\text { discharge), } 60 \\
\mathrm{~mA} \mathrm{~h} \mathrm{~g}^{-1} \\
\text { (second } \\
\text { charge), } 80 \mathrm{~mA} \\
\mathrm{~h} \mathrm{~g}^{-1} \text { (second } \\
\text { discharge) }\end{array}$ & $\begin{array}{l}\text { b) } \mathrm{Mg}_{1.67} \mathrm{Mo}_{3} \mathrm{O}_{8} \\
\text { (charged) } \\
\text { (Rietveld } \\
\text { refinement) }\end{array}$ & S79 \\
\hline
\end{tabular}




\begin{tabular}{|c|c|c|c|c|c|c|}
\hline Material & $\begin{array}{c}\text { Cycling } \\
\text { conditions: } \\
\text { Temperature, } \\
\text { anode, and } \\
\text { electrolyte } \\
\end{array}$ & $\begin{array}{l}\text { Magnesiated } \\
\text { Product phases }\end{array}$ & $\begin{array}{c}\text { Redox } \\
\text { changes }\end{array}$ & $\begin{array}{c}\text { Reported } \\
\text { Electrochemical } \\
\text { Capacity }\end{array}$ & $\begin{array}{c}\text { Experimentally Verified } \\
\text { Magnesiated } \\
\text { Composition }\end{array}$ & Reference \\
\hline $\mathrm{Mg}_{0.67} \mathrm{Ni}_{1.33} \mathrm{O}_{2}$ & $\begin{array}{c}\text { Room } \\
\text { temperature, } \\
\mathrm{Mg} \text { foil, } 1 \mathrm{M} \\
\mathrm{Mg}\left(\mathrm{ClO}_{4}\right)_{2} \text { in } \\
\text { acetonitrile. }\end{array}$ & $\begin{array}{l}\text { Possible lattice } \\
\text { contraction on } \\
\text { charge (XRD) }\end{array}$ & $\begin{array}{c}\text { Not } \\
\text { provided }\end{array}$ & Not provided & $\begin{array}{l}\text { Not reported, but } \mathrm{Mg} \\
\text { extraction verified by } \\
\text { observed } \mathrm{Mg} \text { plating in a Li-ion } \\
\text { cell, along with } \mathrm{Ni} \text { (implying } \mathrm{Ni} \\
\text { is co-extracted) }\end{array}$ & S5 \\
\hline $\mathrm{Mg}_{0.67} \mathrm{Ni}_{1.33} \mathrm{O}_{2}$ & $\begin{array}{c}70{ }^{\circ} \mathrm{C}, \\
\text { capacitive } \\
\text { carbon, } 1 \mathrm{M} \\
\mathrm{Mg}(\mathrm{TFSA})_{2} \text { in } \\
\text { adiponitrile }\end{array}$ & Not provided & $\begin{array}{c}\text { Not } \\
\text { provided }\end{array}$ & $\begin{array}{c}65 \mathrm{~mA} \mathrm{~h} \mathrm{~g}^{-1} \\
\text { (first cycle), } 40 \\
\mathrm{~mA} \mathrm{~h} \mathrm{~g}^{-1} \text { (cycle } \\
\text { 30) }\end{array}$ & Not provided & S10 \\
\hline
\end{tabular}


Nickel oxides

\begin{tabular}{|c|c|c|c|c|c|c|}
\hline Material & $\begin{array}{c}\text { Cycling } \\
\text { conditions: } \\
\text { Temperature, } \\
\text { anode, and } \\
\text { electrolyte } \\
\end{array}$ & $\begin{array}{l}\text { Magnesiated } \\
\text { Product phases }\end{array}$ & $\begin{array}{l}\text { Redox } \\
\text { changes }\end{array}$ & $\begin{array}{c}\text { Reported } \\
\text { Electrochemical } \\
\text { Capacity }\end{array}$ & $\begin{array}{l}\text { Experimentally Verified } \\
\text { Magnesiated } \\
\text { Composition }\end{array}$ & Reference \\
\hline $\begin{array}{l}\mathrm{Li}_{0.13} \mathrm{Mn}_{0.54} \mathrm{Ni}_{0.13} \mathrm{Co}_{0.13} \mathrm{O}_{2-\delta} \\
\text { (mixed } C 2 / m \text { and } R \overline{3} m \\
\quad \text { layered structures) }\end{array}$ & $\begin{array}{c}60{ }^{\circ} \mathrm{C}, \mathrm{AZ31} \\
\mathrm{Mg}: \mathrm{Al}: \mathrm{Zn}= \\
96: 3: 1 \text { alloy, } \\
0.5 \mathrm{M} \\
\mathrm{Mg}(\mathrm{TFSI})_{2} \text { in } \\
\text { acetonitrile }\end{array}$ & $\begin{array}{l}\text { Mg found to } \\
\text { partially insert } \\
\text { into transition } \\
\text { metal layer of } \\
\text { NMC layered } \\
\text { compound on } \\
\text { discharge, } \\
\text { contributing to } \\
\text { capacity fade. } \\
\text { Expansion of } \\
\text { both } C 2 / m \text { and } \\
R \overline{3} m \text { lattice } \\
\text { structures on } \\
\text { discharge. Mg } \\
\text { preferentially } \\
\text { inserted into the } \\
R \overline{3} m \text { structure. } \\
\text { Minor impurities } \\
\text { formed on } \\
\text { discharge (not } \\
\text { characterized) } \\
\text { (XRD) }\end{array}$ & $\begin{array}{c}\text { Reduction } \\
\text { of } \mathrm{Mn}^{4+} \text { to } \\
\mathrm{Mn}^{3+}, \mathrm{Co}^{3+} \\
\text { to } \mathrm{Co}^{2+} \\
(\text { partial), } \\
\text { and } \mathrm{Ni}^{4+} \text { to } \\
\mathrm{Ni}^{2+} \text { on } \\
\text { discharge } \\
\text { (XAS) }\end{array}$ & $\begin{array}{c}275 \text { mA h g-1 } \\
\text { (first } \\
\text { discharge), } 80 \\
\text { mA h g-1 (cycle } \\
\text { 3) }\end{array}$ & $\begin{array}{l}\mathrm{Mg}_{0.43} \mathrm{Li}_{0.13} \mathrm{Mn}_{0.54} \mathrm{Ni}_{0.13} \mathrm{Co}_{0.13} \mathrm{O}_{2-\delta} \\
\text { (Rietveld refinement of XRD) }\end{array}$ & S80 \\
\hline
\end{tabular}


Nickel oxides

\begin{tabular}{|c|c|c|c|c|c|c|}
\hline Material & $\begin{array}{c}\text { Cycling } \\
\text { conditions: } \\
\text { Temperature, } \\
\text { anode, and } \\
\text { electrolyte }\end{array}$ & $\begin{array}{l}\text { Magnesiated } \\
\text { Product phases }\end{array}$ & $\begin{array}{l}\text { Redox } \\
\text { changes }\end{array}$ & $\begin{array}{c}\text { Reported } \\
\text { Electrochemical } \\
\text { Capacity }\end{array}$ & $\begin{array}{c}\text { Experimentally Verified } \\
\text { Magnesiated } \\
\text { Composition }\end{array}$ & Reference \\
\hline $\begin{array}{l}\text { a) } \mathrm{Li}_{0.45} \mathrm{Ni}_{0.5} \mathrm{Mn}_{0.5} \mathrm{O}_{2}, \text { b) } \\
\mathrm{Li}_{0.28} \mathrm{Ni}_{0.5} \mathrm{Mn}_{0.5} \mathrm{O}_{2}\end{array}$ & $\begin{array}{c}60^{\circ} \mathrm{C}, \mathrm{AZ31} \\
0.5 \mathrm{M} \\
\mathrm{Mg}(\mathrm{TFSA}) 2 \text { in } \\
\text { acetonitrile }\end{array}$ & $\begin{array}{l}\mathrm{Mg}^{2+} \text { situated on } \\
\text { 3a positions. } \\
\text { Elongation of the } \\
a \text { axis and } \\
\text { contraction of } \\
\text { the } c \text { axis with } \\
\text { discharge. (XRD) }\end{array}$ & $\begin{array}{c}\text { a) } \\
\text { Reduction } \\
\text { of Ni with } \\
\text { discharge, } \\
\text { the redox } \\
\text { state of } \mathrm{Mn} \\
\text { did not } \\
\text { change } \\
\text { (XAS) }\end{array}$ & $\begin{array}{c}\text { a) } 100 \mathrm{~mA} \mathrm{~h} \mathrm{~g}^{-1} \\
\text { (first } \\
\text { discharge), } 180 \\
\mathrm{~mA} \mathrm{~h} \mathrm{~g} \text { (cycle } \\
\text { 5) } \\
\text { b) } 129 \mathrm{~mA} \mathrm{~h} \mathrm{~g}{ }^{-1} \\
\text { (first } \\
\text { discharge), } 185 \\
\mathrm{~mA} \mathrm{~h} \mathrm{~g}{ }^{-1} \text { (cycle } \\
\text { 5) }\end{array}$ & $\begin{array}{l}\text { a) } \mathrm{Mg}_{0.06} \mathrm{Li}_{0.45} \mathrm{Ni}_{0.5} \mathrm{Mn}_{0.5} \mathrm{O}_{2} \\
\text { (Rietveld refinement) }\end{array}$ & S81 \\
\hline
\end{tabular}


Tin oxides

\begin{tabular}{|c|c|c|c|c|c|c|}
\hline Material & $\begin{array}{l}\text { Cycling conditions: } \\
\text { Temperature, anode, } \\
\text { and electrolyte }\end{array}$ & $\begin{array}{l}\text { Magnesiated } \\
\text { Product phases }\end{array}$ & $\begin{array}{l}\text { Redox } \\
\text { changes }\end{array}$ & $\begin{array}{c}\text { Reported } \\
\text { Electrochemical } \\
\text { Capacity }\end{array}$ & $\begin{array}{l}\text { Experimentally } \\
\text { Verified } \\
\text { Magnesiated } \\
\text { Composition }\end{array}$ & Reference \\
\hline $\begin{array}{l}\text { a) Pure } \mathrm{SnO}_{2} \text {, b) } \mathrm{SnO}_{2} \text { on } \\
\text { reduced graphene oxide }\end{array}$ & $\begin{array}{c}\text { Room temperature, } \\
\mathrm{Mg} \text { foil, } 0.4 \mathrm{M} \mathrm{APC} \\
\text { electrolyte ( } \mathrm{PhMgCl} \\
\text { and } \mathrm{AlCl}_{3} \text { in } 2: 1 \mathrm{molar} \\
\text { ratio) in } \mathrm{THF}\end{array}$ & Not provided & Not provided & 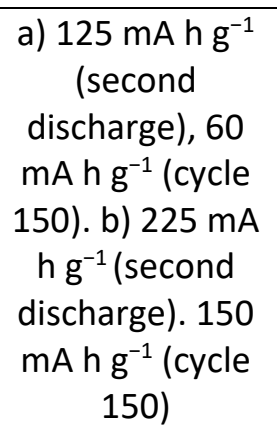 & Not provided & S82 \\
\hline
\end{tabular}


Titanium oxides

\begin{tabular}{|c|c|c|c|c|c|c|}
\hline Material & $\begin{array}{c}\text { Cycling } \\
\text { conditions: } \\
\text { Temperature, } \\
\text { anode, and } \\
\text { electrolyte }\end{array}$ & $\begin{array}{l}\text { Magnesiated } \\
\text { Product phases }\end{array}$ & Redox changes & $\begin{array}{c}\text { Reported } \\
\text { Electrochemical } \\
\text { Capacity }\end{array}$ & $\begin{array}{l}\text { Experimentally } \\
\text { Verified } \\
\text { Magnesiated } \\
\text { Composition }\end{array}$ & Reference \\
\hline $\begin{array}{l}\text { a) } \mathrm{Mg}_{0.5} \mathrm{Ti}_{2}\left(\mathrm{PO}_{4}\right)_{3} \\
\text { b) } \mathrm{MgFeTi}\left(\mathrm{PO}_{4}\right)_{3} \\
\text { c) } \mathrm{MgCrTi}\left(\mathrm{PO}_{4}\right)_{3}\end{array}$ & $\begin{array}{l}\text { Room } \\
\text { temperature, } \mathrm{Mg} \\
\text { foil, } 1 \mathrm{M} \\
\mathrm{Mg}\left(\mathrm{ClO}_{4}\right)_{2} \text { in } \mathrm{PC}\end{array}$ & $\begin{array}{l}\text { a - c) Little or no } \\
\text { change observed } \\
\text { in diffraction } \\
\text { pattern or unit } \\
\text { cell parameters } \\
\text { (XRD) }\end{array}$ & Not provided & $\begin{array}{l}\text { a) } 220 \mathrm{~mA} \mathrm{~h} \mathrm{~g}^{-1} \\
\text { (first discharge). } \\
\text { b) } 180 \mathrm{~mA} \mathrm{~h} \mathrm{~g}^{-1} \\
\text { (first discharge). } \\
\text { c) } 170 \mathrm{~mA} \mathrm{~h} \mathrm{~g}^{-1} \\
\text { (first discharge) }\end{array}$ & Not provided & S83-85 \\
\hline $\begin{array}{l}\text { a) } \\
\mathrm{Na}_{0.24} \mathrm{~V}_{1.25} \mathrm{Ti}_{0.75} \mathrm{O}_{4} \\
\text { b) } \\
\mathrm{Na}_{0.4} \mathrm{~V}_{1.25} \mathrm{Ti}_{0.75} \mathrm{O}_{4}\end{array}$ & $\begin{array}{l}60^{\circ} \mathrm{C}, \mathrm{Mg} \text { foil, } 0.5 \\
\mathrm{M} \mathrm{Mg}\left(\mathrm{CB}_{11} \mathrm{H}_{12}\right)_{2} \text { in } \\
\text { tetraglyme }\end{array}$ & $\begin{array}{l}\text { Mg-Na ion } \\
\text { exchange } \\
\text { experiments } \\
\text { (chemical) gave } \\
\text { lattice } \\
\text { contraction with } \\
\text { minor } \mathrm{Mg} \\
\text { incorporation (Mg } \\
<0.41 \text { per } \\
\text { formula unit } \\
\text { Large } \mathrm{Mg} \\
\text { substitution gave } \\
\text { complete } \\
\text { structural } \\
\text { transformation to } \\
\mathrm{MgM}_{2} \mathrm{O}_{5} \text { and } \\
\mathrm{MgMO}_{3} \text { Oxides ( } \mathrm{M} \\
=\mathrm{Ti}_{1} \mathrm{~V} \text { ) (XRD) } \\
\end{array}$ & $\begin{array}{l}\text { Ti was redox } \\
\text { inactive. } \mathrm{V}^{3.2+} \text { was } \\
\text { reduced to } \mathrm{V}^{4+} \\
\text { when } \\
\text { electrochemically } \\
\text { charged, and was } \\
\text { partially reduced } \\
\text { back when } \\
\text { chemically } \\
\text { magnesiated } \\
\text { (XANES) }\end{array}$ & $\begin{array}{l}\text { a) } 85 \mathrm{~mA} \mathrm{~h} \mathrm{~g}^{-1} \\
\text { (first discharge), } \\
35 \mathrm{~mA} \mathrm{~h} \mathrm{~g}^{-1} \text { (cycle } \\
\text { 3). b) } 65 \mathrm{~mA} \mathrm{~h} \mathrm{~g}^{-1} \\
\text { (first discharge), } \\
15 \mathrm{~mA} \mathrm{~h} \mathrm{~g}^{-1} \text { (cycle } \\
\text { 3). }\end{array}$ & $\begin{array}{l}\text { a) } \\
\mathrm{Mg}_{0.12(2)} \mathrm{Na}_{x} \mathrm{~V}_{1.25} \mathrm{Ti}_{0} \\
.75 \mathrm{O}_{4} \text { on discharge } \\
\text { (EDX). b) Not } \\
\text { provided }\end{array}$ & S86 \\
\hline
\end{tabular}


Titanium oxides

\begin{tabular}{|c|c|c|c|c|c|c|}
\hline Material & $\begin{array}{c}\text { Cycling } \\
\text { conditions: } \\
\text { Temperature, } \\
\text { anode, and } \\
\text { electrolyte }\end{array}$ & $\begin{array}{l}\text { Magnesiated } \\
\text { Product phases }\end{array}$ & Redox changes & $\begin{array}{l}\text { Reported } \\
\text { Electrochemical } \\
\text { Capacity }\end{array}$ & $\begin{array}{l}\text { Experimentally } \\
\text { Verified } \\
\text { Magnesiated } \\
\text { Composition }\end{array}$ & Reference \\
\hline $\mathrm{Li}_{4} \mathrm{Ti}_{5} \mathrm{O}_{12}$ & $\begin{array}{l}\text { Room } \\
\text { temperature, } \mathrm{Mg} \\
\text { foil, } 0.5 \mathrm{M} \\
\mathrm{Mg}(\mathrm{TFSI})_{2}+0.13 \\
\mathrm{M} \mathrm{MgCl} \cdot 6 \mathrm{H}_{2} \mathrm{O} \text { in } \\
\mathrm{DME}\end{array}$ & $\begin{array}{l}\text { No unit cell } \\
\text { changes with } 233 \\
\mathrm{~mA} \mathrm{~h}^{-1} \\
\text { discharge, slight } \\
\text { unit cell } \\
\text { expansion with } \\
\text { discharge to } 290 \\
\mathrm{~mA} \mathrm{~h}^{-1} \text {. } \\
\text { Formation of a } \\
\text { new phase (not } \\
\text { identified) } \\
\text { indicated by a } \\
\text { single new } \\
\text { diffraction peak. } \\
\text { (XRD) }\end{array}$ & $\begin{array}{l}\sim 65 \% \text { of surface } \\
\mathrm{Ti}^{4+} \text { was reduced } \\
\text { to } \mathrm{Ti}^{3+} \text { on } \\
\text { discharge to } 290 \\
\mathrm{~mA} \mathrm{~h} \mathrm{~g}^{-1} \text {. (XPS) }\end{array}$ & $\begin{array}{l}290 \mathrm{~mA} \mathrm{~h} \mathrm{~g}^{-1} \text { (first } \\
\text { discharge), } 40 \mathrm{~mA} \\
\mathrm{~h} \mathrm{~g}^{-1} \text { (cycle 3) }\end{array}$ & Not provided & S87 \\
\hline $\begin{array}{l}\text { a) } \mathrm{Li}_{4} \mathrm{Ti}_{5} \mathrm{O}_{12} \\
\text { b) } \\
\mathrm{Li}_{3.91} \mathrm{Cr}_{0.26} \mathrm{Ti}_{4.82} \mathrm{O}_{12}\end{array}$ & $\begin{array}{l}\text { Room } \\
\text { temperature, } \mathrm{Mg} \\
\text { foil, } 1 \mathrm{M} \text { Tris }(2 \mathrm{H}- \\
\text { hexafluoroisopro } \\
\text { pyl)borate and } \\
0.048 \mathrm{MgF}_{2} \text { in } \\
\text { DME }\end{array}$ & Not provided & Not provided & $\begin{array}{l}\text { a) } 45 \mathrm{~mA} \mathrm{~h} \mathrm{~g}^{-1} \\
\text { (first discharge), } \\
40 \mathrm{~mA} \mathrm{~h} \mathrm{~g}^{-1} \text { (cycle } \\
\text { 5) } \\
\text { b) } 130 \mathrm{~mA} \mathrm{~h} \mathrm{~g}^{-1} \\
\text { (first discharge), } \\
110 \mathrm{~mA} \mathrm{~h} \mathrm{~g}{ }^{-1} \\
\text { (cycle 5) }\end{array}$ & $\begin{array}{l}\text { a) } \mathrm{Li}_{\times} \mathrm{Mg}_{0.4} \mathrm{Ti}_{5} \mathrm{O}_{12} \\
\text { (up to } 10 \mathrm{~nm} \\
\text { below surface). } \\
\text { No } \mathrm{Mg} \text { found } \\
\text { beyond first } 10 \\
\mathrm{~nm} \text {. } \\
\text { b) } \\
\mathrm{Li}_{x} \mathrm{Mg}_{0.84} \mathrm{Cr}_{0.26} \mathrm{Ti}_{4.82} \\
\mathrm{O}_{12} \text { (up to at least } \\
30 \mathrm{~nm} \text { below } \\
\text { surface (XPS } \\
\text { depth profiling) }\end{array}$ & S88 \\
\hline
\end{tabular}


Titanium oxides

\begin{tabular}{|c|c|c|c|c|c|c|}
\hline Material & $\begin{array}{c}\text { Cycling } \\
\text { conditions: } \\
\text { Temperature, } \\
\text { anode, and } \\
\text { electrolyte }\end{array}$ & $\begin{array}{l}\text { Magnesiated } \\
\text { Product phases }\end{array}$ & Redox changes & $\begin{array}{c}\text { Reported } \\
\text { Electrochemical } \\
\text { Capacity }\end{array}$ & $\begin{array}{l}\text { Experimentally } \\
\text { Verified } \\
\text { Magnesiated } \\
\text { Composition }\end{array}$ & Reference \\
\hline $\mathrm{Li}_{4} \mathrm{Ti}_{5} \mathrm{O}_{12}$ & $\begin{array}{l}\text { Room } \\
\text { temperature, } \mathrm{Mg} \\
\text { foil, } 0.25 \mathrm{M} \\
\mathrm{Mg}\left(\mathrm{AlCl}_{2} \mathrm{BuEt}_{2}\right)_{2} \text { in } \\
\mathrm{THF}\end{array}$ & $\begin{array}{l}\text { No peak shifts or } \\
\text { new crystalline } \\
\text { phases observed } \\
\text { during cycling } \\
\text { (XRD) Suggested } \\
\text { Mg occupation on } \\
\text { the 16c site on } \\
\text { discharge due to } \\
\text { increased } \\
\text { contrast in } \\
\text { HAADF-STEM. } \\
\text { Extraction of } \\
\text { some Mg from } \\
\text { the 16c site, and } \\
\text { Mg occupation of } \\
\text { the } 8 \text { a sites, on } \\
\text { charge. } \\
\text { Magnesiation/de } \\
\text { magnesiation } \\
\text { leads to the } \\
\text { formation of } \\
\text { multiple spinel } \\
\text { phases with } \\
\text { different Mg } \\
\text { occupancies on } \\
\text { different sites } \\
\text { (HAADF-STEM). }\end{array}$ & Not provided & $\begin{array}{l}160 \mathrm{~mA} \mathrm{~h} \mathrm{~g}^{-1} \text { (first } \\
\text { discharge), } 158 \\
\mathrm{~mA} \mathrm{~h} \mathrm{~g}^{-1} \text { (cycle } \\
100 \text { ) }\end{array}$ & $\begin{array}{l}\mathrm{Mg}_{4} \mathrm{LiTi}_{5} \mathrm{O}_{12} \\
\text { (discharged), } \\
\mathrm{Mg}_{2.5} \mathrm{LiTi}_{5} \mathrm{O}_{12} \\
\text { (charged) (ICP- } \\
\text { AES) }\end{array}$ & $\mathrm{S} 89,90$ \\
\hline
\end{tabular}




\begin{tabular}{|c|c|c|c|c|c|c|}
\hline Material & $\begin{array}{l}\text { Cycling conditions: } \\
\text { Temperature, } \\
\text { anode, and } \\
\text { electrolyte }\end{array}$ & $\begin{array}{l}\text { Magnesiated } \\
\text { Product phases }\end{array}$ & Redox changes & $\begin{array}{l}\text { Reported } \\
\text { Electrochemical } \\
\text { Capacity }\end{array}$ & $\begin{array}{l}\text { Experimentally } \\
\text { Verified } \\
\text { Magnesiated } \\
\text { Composition }\end{array}$ & Reference \\
\hline $\mathrm{WO}_{3}$ & $\begin{array}{l}\text { Room temperature, } \\
\mathrm{Mg} \text { foil, } 1 \mathrm{M} \\
\mathrm{Mg}\left(\mathrm{ClO}_{4}\right)_{2} \text { in THF }\end{array}$ & Not provided & Not provided & $\begin{array}{l}116 \mathrm{~mA} \mathrm{~h} \mathrm{~g}^{-1} \\
\text { (first discharge) }\end{array}$ & Not provided & S91 \\
\hline a) $\mathrm{WO}_{3}$, b) $\mathrm{WO}_{3} \cdot \mathrm{H}_{2} \mathrm{O}$ & $\begin{array}{l}\text { Room temperature, } \\
\mathrm{Ag} \text { wire, } 0.1 \mathrm{M} \\
\mathrm{Mg}\left(\mathrm{ClO}_{4}\right)_{2} \text { in } \\
\text { acetonitrile }\end{array}$ & $\begin{array}{l}\text { a) and b) No } \\
\text { change in local } \\
\text { structure on } \\
\text { discharge (Raman } \\
\text { spectroscopy). a) } \\
\text { Change of unit cell } \\
\text { to cubic structure } \\
\text { (from monoclinic) } \\
\text { on discharge, } \\
\text { reverts to } \\
\text { monoclinic on } \\
\text { charge (XRD) }\end{array}$ & $\begin{array}{l}\text { a) and b) Thin } \\
\text { films display } \\
\text { electrochromism, } \\
\text { indicative of } \\
\text { reduction of } \\
\text { Mo }^{6+} \text { with } \\
\text { discharge }\end{array}$ & $\begin{array}{l}\text { a) } 75 \mathrm{~mA} \mathrm{~h} \mathrm{~g}^{-1} \\
\text { (unknown } \\
\text { cycle). b) } 33 \mathrm{~mA} \\
\mathrm{~h} \mathrm{~g}^{-1} \text { (unknown } \\
\text { cycle). }\end{array}$ & $\begin{array}{l}\text { Presence of Mg } \\
\text { verified by EDS } \\
\text { (was not } \\
\text { quantified) }\end{array}$ & S92 \\
\hline $\begin{array}{l}\left.\text { a) } \mathrm{K}_{0.26} \mathrm{~W}_{3} \mathrm{O}_{9}, \mathrm{~b}\right) \\
\left.\mathrm{K}_{0.26}\left(\mathrm{~W}_{0.9} \mathrm{Mo}_{0.1}\right)_{3} \mathrm{O}_{9}, \mathrm{c}\right) \\
\mathrm{K}_{0.26}\left(\mathrm{~W}_{0.95} \mathrm{Cr}_{0.05}\right)_{3} \mathrm{O}_{9}\end{array}$ & 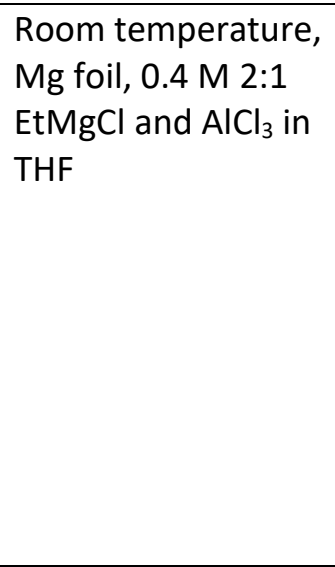 & $\begin{array}{l}\text { b) Lattice peak } \\
\text { shifts and } \\
\text { broadening on } \\
\text { discharge (XRD) }\end{array}$ & Not provided & $\begin{array}{l}\text { a) } 2 \mathrm{~mA} \mathrm{~h} \mathrm{~g}^{-1} \\
\text { (constant } \\
\text { current), } 5 \mathrm{~mA} \\
\mathrm{~h} \mathrm{~g}^{-1} \text { (volt hold), } \\
\text { b) } 5 \mathrm{~mA} \mathrm{~h} \mathrm{~g}^{-1} \\
\text { (constant } \\
\text { current), } 14 \mathrm{~mA} \\
\mathrm{~h} \mathrm{~g}^{-1} \text { (volt hold) } \\
\text { c) } 5 \mathrm{~mA} \mathrm{~h} \mathrm{~g} \\
\text { (constant } \\
\text { current), } 11 \mathrm{~mA} \\
\mathrm{~h} \mathrm{~g}^{-1} \text { (volt hold) }\end{array}$ & $\begin{array}{l}\text { Mg evident in } \\
\text { discharged } \\
\text { sample } \\
\text { (quantification } \\
\text { not provided) } \\
\text { (EDS) }\end{array}$ & S93 \\
\hline
\end{tabular}




\begin{tabular}{|c|c|c|c|c|c|c|}
\hline Material & $\begin{array}{c}\text { Cycling } \\
\text { conditions: } \\
\text { Temperature, } \\
\text { anode, and } \\
\text { electrolyte }\end{array}$ & $\begin{array}{l}\text { Magnesiated Product } \\
\text { phases }\end{array}$ & Redox changes & $\begin{array}{c}\text { Reported } \\
\text { Electrochemical } \\
\text { Capacity }\end{array}$ & $\begin{array}{c}\text { Experimentally } \\
\text { Verified Magnesiated } \\
\text { Composition }\end{array}$ & Reference \\
\hline$\alpha-\mathrm{V}_{2} \mathrm{O}_{5}$ & $\begin{array}{l}150{ }^{\circ} \mathrm{C} \text {, Li foil, a) } 1 \\
\mathrm{~mol} \mathrm{~kg}^{-1} \\
\mathrm{Mg}\left(\mathrm{ClO}_{4}\right)_{2} \text { in } \\
\mathrm{DMSO}_{2}, \text { b) } 0.7 \mathrm{~mol} \\
\mathrm{~kg}^{-1} \mathrm{Mg}\left(\mathrm{CF}_{3} \mathrm{SO}_{3}\right)_{2} \\
\text { in } \mathrm{TMSO}_{2} \text {. }\end{array}$ & $\begin{array}{l}\text { Small expansion of } \\
\text { unit cell volume with } \\
\text { discharge (XRD). }\end{array}$ & Not provided & $\begin{array}{l}\text { a) and b) } 140 \\
m A ~^{-1} \text { (first } \\
\text { discharge) }\end{array}$ & Not provided & S94 \\
\hline $\begin{array}{l}\text { a) } \mathrm{V}_{2} \mathrm{O}_{5} \text { Xerogel, b) } \mathrm{V}_{2} \mathrm{O}_{5} \\
\text { Xerogel/C composite }\end{array}$ & $\begin{array}{l}\text { Room } \\
\text { temperature, } \mathrm{Pt} \\
\text { foil, saturated } \\
\text { solution of } \\
\mathrm{Mg}\left(\mathrm{NO}_{3}\right)_{2} \text { in water }\end{array}$ & Not provided & Not provided & $\begin{array}{l}\text { a) } 50 \mathrm{~mA} \mathrm{~h} \mathrm{~g}^{-1} \\
\text { (unknown } \\
\text { cycle). b) } 107 \\
\mathrm{~mA} \mathrm{~h} \mathrm{~g}^{-1} \\
\text { (unknown } \\
\text { cycle) }\end{array}$ & Not provided & S95 \\
\hline$\alpha-V_{2} O_{5}$ & $\begin{array}{l}\text { Room } \\
\text { temperature, } \mathrm{Mg} \\
\text { foil, } 1 \mathrm{M} \\
\mathrm{Mg}\left(\mathrm{ClO}_{4}\right)_{2} \text { in a) } \\
<1000 \mathrm{ppm} \mathrm{H}_{2} \mathrm{O} \\
\text { acetonitrile or b) } \\
\text { acetonitrile with } \\
0.9 \text { or } 1 \mathrm{M} \text { water } \\
\text { added }\end{array}$ & $\begin{array}{l}\text { XRD of a discharged } \\
\text { single crystal } \\
\left(\mathrm{Mg}_{0.01} \mathrm{~V}_{2} \mathrm{O}_{5}\right) \\
\text { suggested a location } \\
\text { for } \mathrm{Mg} \text { in the lattice } \\
(\mathrm{XRD}) \text {. Delamination } \\
\text { of a } \mathrm{V}_{2} \mathrm{O}_{5} \text { single crystal } \\
\text { observed following } \\
\text { discharge (SEM) Slight } \\
\text { contraction of the unit } \\
\text { cell on discharge } \\
\text { (XRD) }\end{array}$ & Not provided & $\begin{array}{l}\text { a) } 25 \mathrm{~mA} \mathrm{~h} \mathrm{~g}^{-1} \\
\text { (first cycle). b) } \\
175 \mathrm{~mA} \mathrm{~h} \mathrm{~g}^{-1} \\
\text { (first cycle) }\end{array}$ & $\begin{array}{l}\text { Amount of Mg } \\
\text { corresponded to } \\
\text { discharge capacity, } \\
\text { values not provided } \\
\text { (AES) }\end{array}$ & S14,96,97 \\
\hline
\end{tabular}




\begin{tabular}{|c|c|c|c|c|c|c|}
\hline Material & $\begin{array}{c}\text { Cycling } \\
\text { conditions: } \\
\text { Temperature, } \\
\text { anode, and } \\
\text { electrolyte }\end{array}$ & $\begin{array}{c}\text { Magnesiated Product } \\
\text { phases }\end{array}$ & Redox changes & $\begin{array}{l}\text { Reported } \\
\text { Electrochemical } \\
\text { Capacity }\end{array}$ & $\begin{array}{c}\text { Experimentally } \\
\text { Verified Magnesiated } \\
\text { Composition }\end{array}$ & Reference \\
\hline $\begin{array}{l}\left.\text { a) } \mathrm{Mg}\left(\mathrm{V}_{3} \mathrm{O}_{8}\right)_{2}, \mathrm{~b}\right) \mathrm{NaV}_{3} \mathrm{O}_{8} \text {, } \\
\text { c) } \mathrm{V}_{2} \mathrm{O}_{5} \text { Xerogel }\end{array}$ & $\begin{array}{l}80{ }^{\circ} \mathrm{C} \text {, Al foil, a } \\
\text { salt melt } \\
\text { containing } 3 \mathrm{w} / \mathrm{o} \\
\mathrm{MgCl}_{2}, 56 \mathrm{w} / \mathrm{o} \\
\mathrm{AlCl}_{3} \text {, and } 41 \mathrm{w} / \mathrm{o} \\
\mathrm{EMIC}\end{array}$ & Not provided & Not provided & $\begin{array}{l}\text { a) } 155 \mathrm{~mA} \mathrm{~h} \mathrm{~g}^{-1} \\
\text { (first } \\
\text { discharge), } 60 \\
\mathrm{~mA} \mathrm{~h} \mathrm{~g}^{-1} \text { (cycle } \\
\text { 50) b) >80 mA h } \\
\mathrm{g}^{-1} \text { (long-term } \\
\text { cycling) c) } 170 \\
\mathrm{~mA} \mathrm{~h}^{-1} \text { (first } \\
\text { cycle), } 60-70 \\
\mathrm{~mA} \mathrm{~h} \mathrm{~g}^{-1} \\
\text { (subsequent } \\
\text { cycles) }\end{array}$ & Not provided & S98 \\
\hline $\begin{array}{l}\mathrm{V}_{2} \mathrm{O}_{5} \text { Xerogel } \\
\left(\mathrm{V}_{2} \mathrm{O}_{5} \cdot 0.4 \mathrm{H}_{2} \mathrm{O}\right)\end{array}$ & $\begin{array}{l}25^{\circ} \mathrm{C} \text {, chemically- } \\
\text { inserted } \mathrm{V}_{2} \mathrm{O}_{5} \\
\left(\mathrm{Mg}_{0.62} \mathrm{~V}_{2} \mathrm{O}_{5}\right), 0.1 \\
\mathrm{M} \mathrm{Mg}\left(\mathrm{CF}_{3} \mathrm{SO}_{3}\right)_{2} \text { in } \\
\mathrm{PC} \text {. }\end{array}$ & Not provided & Not provided & 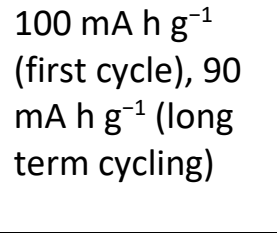 & Not provided & S99 \\
\hline$\alpha-\mathrm{V}_{2} \mathrm{O}_{5}$ & $\begin{array}{l}\text { Room } \\
\text { temperature, } \\
\text { capacitive carbon, } \\
0.5 \mathrm{M} \mathrm{Mg}\left(\mathrm{ClO}_{4}\right)_{2} \\
\text { in } \mathrm{PC}(18 \mathrm{ppm} \\
\text { water) }\end{array}$ & Not provided & Not provided & $\begin{array}{l}260 \mathrm{~mA} \mathrm{~h} \mathrm{~g}^{-1} \\
\text { (first } \\
\text { discharge), } 190 \\
\mathrm{~mA} \mathrm{~h} \mathrm{~g}^{-1} \text { (cycle } \\
\text { 2) }\end{array}$ & Not provided & S100 \\
\hline
\end{tabular}




\begin{tabular}{|c|c|c|c|c|c|c|}
\hline Material & $\begin{array}{c}\text { Cycling } \\
\text { conditions: } \\
\text { Temperature, } \\
\text { anode, and } \\
\text { electrolyte } \\
\end{array}$ & $\begin{array}{c}\text { Magnesiated Product } \\
\text { phases }\end{array}$ & Redox changes & $\begin{array}{c}\text { Reported } \\
\text { Electrochemical } \\
\text { Capacity }\end{array}$ & $\begin{array}{c}\text { Experimentally } \\
\text { Verified Magnesiated } \\
\text { Composition }\end{array}$ & Reference \\
\hline $\mathrm{V}_{2} \mathrm{O}_{5}$ Xerogel/C & $\begin{array}{l}\text { Room } \\
\text { temperature, } \mathrm{Mg} \\
\text { foil, } 1 \mathrm{M} \\
\mathrm{Mg}\left(\mathrm{ClO}_{4}\right)_{2} \text { in } \\
\text { acetonitrile }\end{array}$ & $\begin{array}{l}\text { Lattice parameters } a \\
\text { and } b \text { expand, } c \\
\text { contracts, with } \\
\text { discharge. Reversed } \\
\text { on charge. (XRD) } \\
\text { Changes observed in } \\
\text { local structure with } \\
\text { discharge, reversed } \\
\text { with charge (FT-IR) }\end{array}$ & Not provided & $\begin{array}{l}445 \mathrm{~mA} \mathrm{~h} \mathrm{~g}^{-1} \\
\text { (first } \\
\text { discharge), } 290 \\
\mathrm{~mA} \mathrm{~h} \mathrm{~g}^{-1} \text { (cycle } \\
\text { 35) }\end{array}$ & $\begin{array}{l}\mathrm{Mg}_{1.84} \mathrm{~V}_{2} \mathrm{O}_{5} \text { on } \\
\text { discharge (ICP-AES) }\end{array}$ & S101,102 \\
\hline$\alpha-\mathrm{V}_{2} \mathrm{O}_{5}$ & $\begin{array}{l}\text { Room } \\
\text { temperature, } \mathrm{Mg} \\
\text { foil, } 0.1 \mathrm{M} \\
\mathrm{Mg}\left(\mathrm{ClO}_{4}\right)_{2} \text { in } \mathrm{PC} \\
\text { with a) no added } \\
\text { water and b) } 1.79 \\
\mathrm{M} \text { water added }\end{array}$ & $\begin{array}{l}\text { b) Destruction of } \alpha- \\
\mathrm{V}_{2} \mathrm{O}_{5} \text { and creation of } \\
\text { new crystalline phases } \\
\text { on discharge (XRD) }\end{array}$ & Not provided & $\begin{array}{l}\text { a) } 60 \mathrm{~mA} \mathrm{~h} \mathrm{~g}^{-1} \\
\text { (first } \\
\text { discharge). b) } \\
160 \mathrm{~mA} \mathrm{~h} \mathrm{~g}{ }^{-1} \\
\text { (first } \\
\text { discharge), } 40 \\
\mathrm{~mA} \mathrm{~h} \mathrm{~g}^{-1} \text { (cycle } \\
\text { 9) }\end{array}$ & Not provided & S103 \\
\hline $\mathrm{V}_{2} \mathrm{O}_{5}$ Xerogel & $\begin{array}{l}25^{\circ} \mathrm{C}, \mathrm{Mg}- \\
\text { inserted } \mathrm{V}_{2} \mathrm{O}_{5} \\
\text { Xerogel, } 0.3 \mathrm{M} \\
\mathrm{Mg}\left(\mathrm{ClO}_{4}\right)_{2} \text { in } \mathrm{PC}\end{array}$ & Not provided & Not provided & $\begin{array}{l}180 \mathrm{~mA} \mathrm{~h} \mathrm{~g}^{-1} \\
\text { (first } \\
\text { discharge), } 400 \\
\mathrm{~mA} \mathrm{~h} \mathrm{~g}^{-1} \text { (cycle } \\
\text { 3) }\end{array}$ & Not provided & S104 \\
\hline
\end{tabular}




\begin{tabular}{|c|c|c|c|c|c|c|}
\hline Material & $\begin{array}{c}\text { Cycling } \\
\text { conditions: } \\
\text { Temperature, } \\
\text { anode, and } \\
\text { electrolyte } \\
\end{array}$ & $\begin{array}{l}\text { Magnesiated Product } \\
\text { phases }\end{array}$ & Redox changes & $\begin{array}{c}\text { Reported } \\
\text { Electrochemical } \\
\text { Capacity }\end{array}$ & $\begin{array}{c}\text { Experimentally } \\
\text { Verified Magnesiated } \\
\text { Composition }\end{array}$ & Reference \\
\hline$\alpha-V_{2} O_{5}$ (thin film) & $\begin{array}{l}\text { Room } \\
\text { temperature, } \\
\text { capacitive carbon, } \\
\text { a) } 0.1 \mathrm{M} \mathrm{Mg}(\mathrm{TFSI})_{2} \\
\text { in acetonitrile }(32 \\
\left.\text { ppm } \mathrm{H}_{2} \mathrm{O}\right) \text { b) } 0.5 \\
\mathrm{M} \mathrm{Mg}\left(\mathrm{ClO}_{4}\right)_{2} \text { in } \\
\text { acetonitrile }(212 \\
\left.\text { ppm } \mathrm{H}_{2} \mathrm{O}\right)\end{array}$ & $\begin{array}{l}\text { b) Expansion of the } \\
\text { interlayer distance ( } b \\
\text { lattice parameter) } \\
\text { with discharge (and } \\
\text { appearance of new } \\
\text { diffraction peaks). } \\
\text { Contraction upon } \\
\text { charge. (XRD) } \\
\text { Increased local } \\
\text { disorder with } \\
\text { discharge (Raman } \\
\text { Spectroscopy) }\end{array}$ & Not provided & $\begin{array}{l}\text { a) } 150 \mathrm{~mA} \mathrm{~h} \mathrm{~g}^{-1} \\
\text { (first } \\
\text { discharge). b) } \\
180 \mathrm{~mA} \mathrm{~h} \mathrm{~g}^{-1} \\
\text { (first } \\
\text { discharge), } 150 \\
\mathrm{~mA} \mathrm{~h} \mathrm{~g}^{-1} \text { (cycle } \\
\text { 36) }\end{array}$ & $\begin{array}{l}\text { b) Mg present in } \\
\text { discharged electrode, } \\
\text { and is not present in } \\
\text { charged electrode } \\
\text { (EDS, quantification } \\
\text { not provided) }\end{array}$ & S75 \\
\hline $\begin{array}{l}\text { Mg-enriched } \mathrm{Mg}_{x} \mathrm{~V}_{2} \mathrm{O}_{5} \\
\text { (from } \mathrm{V}_{2} \mathrm{O}_{5} \cdot 0.6 \mathrm{H}_{2} \mathrm{O} \text { ) }\end{array}$ & $\begin{array}{l}\text { Room } \\
\text { temperature, Sn } \\
\text { metal, } 1 \mathrm{M} \\
\mathrm{Mg}\left(\mathrm{ClO}_{4}\right)_{2} \text { in } \\
\text { acetonitrile }\end{array}$ & $\begin{array}{l}\text { Contraction of the } \\
\text { layer axis ( } c \text { lattice } \\
\text { parameter) with } \\
\text { discharge. Expansion } \\
\text { upon charge. (SAXS } \\
\text { and WAXS) }\end{array}$ & $\begin{array}{l}\text { Oxidation of } \mathrm{V}^{5+} \\
\left(\mathrm{V}_{2} \mathrm{O}_{5}\right) \text { to } \mathrm{V}^{4+} \\
\left(\mathrm{Mg}_{x} \mathrm{~V}_{2} \mathrm{O}_{5}\right) \text { on } \\
\text { electrochemical } \\
\text { enrichment. } \\
\text { Oxidation to } \mathrm{V}^{4.8+} \\
\text { on charge, } \\
\text { reduction to } \mathrm{V}^{4+} \text { on } \\
\text { discharge (XANES) }\end{array}$ & $\begin{array}{l}210 \mathrm{~mA} \mathrm{~h} \mathrm{~g}^{-1} \\
\text { (first charge), } \\
155 \mathrm{~mA} \mathrm{~h} \mathrm{~g}^{-1} \\
\text { (cycle 2) }\end{array}$ & $\begin{array}{l}\mathrm{Mg}_{0.7} \mathrm{~V}_{2} \mathrm{O}_{5} \cdot \mathrm{xH}_{2} \mathrm{O} \\
\text { (discharged, enriched } \\
\text { sample), } \\
\mathrm{Mg}_{0.25} \mathrm{~V}_{2} \mathrm{O}_{5} \cdot \mathrm{xH}_{2} \mathrm{O} \\
\text { (charged). (XRF) }\end{array}$ & S105 \\
\hline $\mathrm{Mg}_{0.1} \mathrm{~V}_{2} \mathrm{O}_{5} \cdot 1.8 \mathrm{H}_{2} \mathrm{O}$ & $\begin{array}{l}\text { Room } \\
\text { temperature, } \mathrm{Pt} \\
\text { foil, } 0.5 \mathrm{M} \\
\mathrm{Mg}\left(\mathrm{ClO}_{4}\right)_{2} \text { in } \\
\text { acetonitrile }\end{array}$ & $\begin{array}{l}\text { No change in } \\
\text { interlayer spacing ( } b \\
\text { lattice parameter) on } \\
\text { discharge/charge } \\
\text { (XRD). }\end{array}$ & Not provided & $\begin{array}{l}300 \mathrm{~mA} \mathrm{~h} \mathrm{~g}^{-1} \\
\text { (first } \\
\text { discharge), } 285 \\
\mathrm{~mA} \mathrm{~h} \mathrm{~g} \text { g }^{-1} \text { (cycle } \\
\text { 7) }\end{array}$ & Not provided & S106 \\
\hline
\end{tabular}




\begin{tabular}{|c|c|c|c|c|c|c|}
\hline Material & $\begin{array}{c}\text { Cycling } \\
\text { conditions: } \\
\text { Temperature, } \\
\text { anode, and } \\
\text { electrolyte } \\
\end{array}$ & $\begin{array}{l}\text { Magnesiated Product } \\
\text { phases }\end{array}$ & Redox changes & $\begin{array}{c}\text { Reported } \\
\text { Electrochemical } \\
\text { Capacity }\end{array}$ & $\begin{array}{c}\text { Experimentally } \\
\text { Verified Magnesiated } \\
\text { Composition }\end{array}$ & Reference \\
\hline $\begin{array}{l}\text { a) } \alpha-\mathrm{V}_{2} \mathrm{O}_{5} / \text { Graphene, b) } \\
\mathrm{V}_{2} \mathrm{O}_{5} \cdot 1.42 \mathrm{H}_{2} \mathrm{O} / \text { Graphene }\end{array}$ & $\begin{array}{l}\text { Room } \\
\text { temperature, } \\
\text { capacitive carbon, } \\
0.5 \mathrm{M} \mathrm{Mg}(\mathrm{TFSI})_{2} \text { in } \\
\text { acetonitrile }\end{array}$ & Not provided & Not provided & $\begin{array}{l}\text { a) } 105 \mathrm{~mA} \mathrm{~h} \mathrm{~g}^{-1} \\
\text { (first } \\
\text { discharge), } 50 \\
\mathrm{~mA} \mathrm{~h} \mathrm{~g}^{-1} \text { (cycle } \\
\text { 30) b) } 210 \mathrm{~mA} \mathrm{~h} \\
\mathrm{~g}^{-1} \text { (first } \\
\text { discharge), } 280 \\
\mathrm{~mA} \mathrm{~h} \mathrm{~g}^{-1} \text { (max } \\
\text { discharge, cycle } \\
\text { 8), } 210 \mathrm{~mA} \mathrm{~h} \mathrm{~g} \\
\text { (cycle } 30 \text { ) }\end{array}$ & Not provided & S107 \\
\hline $\begin{array}{l}\text { Amorphous } \mathrm{V}_{2} \mathrm{O}_{5}: \mathrm{P}_{2} \mathrm{O}_{5} \text { in } \\
\text { a } 75: 25 \text { mol\% ratio }\end{array}$ & $\begin{array}{l}\text { Room } \\
\text { temperature, } \mathrm{Mg} \\
\text { foil, } 1 \mathrm{M} \\
\mathrm{Mg}\left(\mathrm{ClO}_{4}\right)_{2} \text { in } \\
\text { acetonitrile }(<50 \\
\left.\text { ppm } \mathrm{H}_{2} \mathrm{O}\right) \text {. }\end{array}$ & Not provided & $\begin{array}{l}\text { Evidence of } \\
\text { reduction of } V \text { with } \\
\text { discharge, and } \\
\text { limited reoxidation } \\
\text { on charge (not } \\
\text { quantified, XPS). }\end{array}$ & 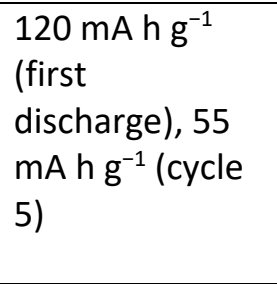 & $\begin{array}{l}\text { Mg present at surface } \\
\text { following discharge, } \\
\text { partially removed on } \\
\text { charge (not quantified, } \\
\text { XPS). }\end{array}$ & S108 \\
\hline$\alpha-\mathrm{V}_{2} \mathrm{O}_{5} /$ Graphite & $\begin{array}{l}\text { Room } \\
\text { temperature, } \mathrm{Pt} \\
\text { foil, } 10.2 \mathrm{M} \\
\mathrm{Mg}\left(\mathrm{NO}_{3}\right)_{2} \text { in water }\end{array}$ & $\begin{array}{l}\text { Some dissolution of } \\
\mathrm{V}_{2} \mathrm{O}_{5} \text { into the } \\
\text { electrolyte (UV/Vis } \\
\text { spectroscopy) }\end{array}$ & Not provided & $\begin{array}{l}88 \mathrm{~mA} \mathrm{~h} \mathrm{~g}^{-1} \\
\text { (first } \\
\text { discharge), } 102 \\
\mathrm{~mA} \mathrm{~h} \mathrm{~g}^{-1} \text { (cycle } \\
\text { 2), } 80 \mathrm{~mA} \mathrm{~h} \mathrm{~g}^{-1} \\
\text { (cycle 10) }\end{array}$ & Not provided & S109 \\
\hline$\alpha-\mathrm{V}_{2} \mathrm{O}_{5} /$ Graphene Oxide & $\begin{array}{l}\text { Room } \\
\text { temperature, } \mathrm{Mg} \\
\text { foil, } 0.25 \mathrm{M} \\
\mathrm{Mg}\left(\mathrm{AlCl}{ }_{2} \mathrm{EtBu}\right)_{2} \text { in } \\
\mathrm{THF}\end{array}$ & Not provided & Not provided & $\begin{array}{l}180 \mathrm{~mA} \mathrm{~h} \mathrm{~g}^{-1} \\
\text { (first } \\
\text { discharge), } 130 \\
\mathrm{~mA} \mathrm{~h} \mathrm{~g}{ }^{-1} \text { (cycle } \\
\text { 20) }\end{array}$ & Not provided & S110 \\
\hline
\end{tabular}




\begin{tabular}{|c|c|c|c|c|c|c|}
\hline Material & $\begin{array}{c}\text { Cycling } \\
\text { conditions: } \\
\text { Temperature, } \\
\text { anode, and } \\
\text { electrolyte }\end{array}$ & $\begin{array}{l}\text { Magnesiated Product } \\
\text { phases }\end{array}$ & Redox changes & $\begin{array}{c}\text { Reported } \\
\text { Electrochemical } \\
\text { Capacity }\end{array}$ & $\begin{array}{c}\text { Experimentally } \\
\text { Verified Magnesiated } \\
\text { Composition }\end{array}$ & Reference \\
\hline $\begin{array}{l}\text { a) } \mathrm{VO}_{2.37}\left[\mathrm{C}_{18} \mathrm{H}_{40} \mathrm{~N}\right]_{0.26} \\
\text { nanotubes }\left(\mathrm{VO}_{\mathrm{x}}-\mathrm{NTs}\right) \text { b) } \\
\text { b) Cu-doped } \mathrm{VO}_{\mathrm{x}}-\mathrm{NTs}\end{array}$ & $\begin{array}{l}\text { Room } \\
\text { temperature, } \mathrm{Mg} \\
\text { foil, } 0.25 \mathrm{M} \\
\mathrm{Mg}\left(\mathrm{AlBu}_{2} \mathrm{Cl}_{2}\right)_{2} / \mathrm{THF}\end{array}$ & $\begin{array}{l}\text { a) Expansion of layer } \\
\text { axis (c lattice } \\
\text { parameter) with } \\
\text { discharge (XRD) }\end{array}$ & $\begin{array}{l}\text { a) Conversion of } \\
\text { surface } \mathrm{V}^{5+} \text { to } \mathrm{V}^{4+} \\
\text { (XPS) }\end{array}$ & $\begin{array}{l}\text { a) } 76 \mathrm{~mA} \mathrm{~h} \mathrm{~g}^{-1} \\
\text { (first discharge) } \\
\text { b) } 120 \mathrm{~mA} \mathrm{~h} \mathrm{~g}^{-1} \\
\text { (first discharge) }\end{array}$ & $\begin{array}{l}\text { a) } \\
\mathrm{Mg}_{0.1} \mathrm{VO}_{2.37}\left[\mathrm{C}_{18} \mathrm{H}_{40} \mathrm{~N}\right]_{0.26} \\
\text { (XPS). Electrochemical } \\
\text { stoichiometry } \\
\text { supported by ICP-AES } \\
\text { (values not given). }\end{array}$ & S111-113 \\
\hline $\mathrm{VO}_{\mathrm{x}}$ Nanotubes & $\begin{array}{l}\text { Room } \\
\text { temperature, } \\
\mathrm{AZ31} \mathrm{Mg} \text { alloy, } 0.5 \\
\mathrm{M} \mathrm{Mg}\left(\mathrm{ClO}_{4}\right)_{2} \text { in } \\
\text { acetonitrile }\end{array}$ & Not provided & $\begin{array}{l}\text { Irreversible surface } \\
\text { oxidation of surface } \\
\mathrm{V}^{3+} \text { to } \mathrm{V}^{4+} \text { and } \mathrm{V}^{5+} \\
\text { after cycling (XPS) }\end{array}$ & $\begin{array}{l}210 \mathrm{~mA} \mathrm{~h} \mathrm{g-1} \\
\text { (first } \\
\text { discharge), } 145 \\
\mathrm{~mA} \mathrm{~h} \mathrm{~g}^{-1} \text { (cycle } \\
\text { 20). }\end{array}$ & Not provided & S114 \\
\hline $\begin{array}{l}\text { Nanocluster } \\
\mathrm{V}_{2} \mathrm{O}_{5} / \text { Porous Carbon }\end{array}$ & $\begin{array}{l}\text { Room } \\
\text { temperature, } \mathrm{Mg} \\
\text { foil, } 0.2 \mathrm{M}\left[\mathrm{Mg}_{2}(\mu-\right. \\
\left.\mathrm{Cl})_{2}(\mathrm{DME})_{4}\right]\left[\mathrm{AlCl}_{4}\right]_{2} \\
\text { in } \mathrm{DME}\end{array}$ & $\begin{array}{l}\text { Decreased intensity } \\
\text { and resolution of }{ }^{51} \mathrm{~V} \text { - } \\
\mathrm{NMR} \text { signal on } \\
\text { discharge, only } \\
\text { partially recovered on } \\
\text { charge, attributed to } \\
\text { formation of localized } \\
V \text { ions ( }{ }^{51} \mathrm{~V} \text {-NMR). } \\
\text { Magnesium vanadate } \\
\text { signature observed in } \\
\text { discharged state } \\
\text { (Raman } \\
\text { Spectroscopy). }\end{array}$ & $\begin{array}{l}\text { Reduction of } \\
\text { surface } \mathrm{V}^{5+} \text { to } \mathrm{V}^{4+} \\
\text { on discharge, } \\
\text { partial reoxidation } \\
\text { on charge (XPS) }\end{array}$ & $\begin{array}{l}340 \mathrm{~mA} \mathrm{~h} \mathrm{~g}^{-1} \\
\text { (first } \\
\text { discharge), } 80 \\
\mathrm{~mA} \mathrm{~h} \mathrm{~g}^{-1} \text { (cycle } \\
\text { 40) }\end{array}$ & $\begin{array}{l}\mathrm{Mg}_{1.5} \mathrm{~V}_{2} \mathrm{O}_{5} \\
\text { (discharged), } \\
\mathrm{Mg}_{0.15} \mathrm{~V}_{2} \mathrm{O}_{5} \text { (charged) } \\
\text { (XPS) EDS showed } \\
\text { increase and decrease } \\
\text { in } \mathrm{Mg} \text { content with } \\
\text { discharge and charge, } \\
\text { respectively (not } \\
\text { quantified). }\end{array}$ & S115 \\
\hline$\beta-\mathrm{NaV}_{6} \mathrm{O}_{15}$ & $\begin{array}{l}\text { Room } \\
\text { temperature, } \mathrm{Mg} \\
\text { foil, } 1 \mathrm{M} \\
\mathrm{Mg}\left(\mathrm{ClO}_{4}\right)_{2} \text { in } \\
\text { acetonitrile }\end{array}$ & Not provided & Not provided & $\begin{array}{l}90 \mathrm{~mA} \mathrm{~h} \mathrm{~g}^{-1} \\
\text { (first } \\
\text { discharge), } 0 \\
\mathrm{~mA} \mathrm{~h}^{-1} \text { (cycle } \\
25 \text { ) }\end{array}$ & Not provided & S116 \\
\hline
\end{tabular}




\begin{tabular}{|c|c|c|c|c|c|c|}
\hline Material & $\begin{array}{c}\text { Cycling } \\
\text { conditions: } \\
\text { Temperature, } \\
\text { anode, and } \\
\text { electrolyte } \\
\end{array}$ & $\begin{array}{c}\text { Magnesiated Product } \\
\text { phases }\end{array}$ & Redox changes & $\begin{array}{c}\text { Reported } \\
\text { Electrochemical } \\
\text { Capacity }\end{array}$ & $\begin{array}{c}\text { Experimentally } \\
\text { Verified Magnesiated } \\
\text { Composition }\end{array}$ & Reference \\
\hline$\alpha-V_{2} O_{5}$ & $\begin{array}{l}\text { Room } \\
\text { temperature, a) } \\
\text { Mg foil or b) } \\
\text { capacitive carbon, } \\
1 \mathrm{M} \mathrm{Mg}(\mathrm{TFSI})_{2} \text { in } \\
\text { diglyme with a) } 15 \\
\text { ppm } \mathrm{H}_{2} \mathrm{O} \text { and b) } \\
2600 \text { ppm } \mathrm{H}_{2} \mathrm{O}\end{array}$ & $\begin{array}{l}\text { a) No protons evident } \\
\text { within } \mathrm{V}_{2} \mathrm{O}_{5} \text { on } \\
\left.\text { discharge ( }{ }^{1} \mathrm{H}-\mathrm{NMR}\right) \text {. } \\
\text { Little change in local } \\
\mathrm{V}_{2} \mathrm{O}_{5} \text { structure on } \\
\text { discharge (EXAFS). A } \\
\text { mixture of } \delta-\mathrm{MgV}_{2} \mathrm{O}_{5} \\
\text { and an unknown new } \\
\text { phase was observed. } \\
\text { b) Protons co- } \\
\text { intercalate into } \mathrm{V}_{2} \mathrm{O}_{5} \\
\text { on discharge }\left({ }^{1} \mathrm{H}_{-}\right. \\
\mathrm{NMR})\end{array}$ & $\begin{array}{l}\text { a). } \mathrm{V}^{5+} \text { partially } \\
\text { reduced to } \mathrm{V}^{4+} \text { on } \\
\text { discharge } \\
\text { (corresponding to } \\
\mathrm{Mg}_{0.2} \mathrm{~V}_{2} \mathrm{O}_{5} \text { ). Charge } \\
\text { led to partial re- } \\
\text { oxidation back to } \\
\mathrm{V}^{5+} \text {. }\end{array}$ & $\begin{array}{l}\text { a). } 82 \mathrm{~mA} \mathrm{~h} \mathrm{~g}^{-1} \\
\text { (first } \\
\text { discharge), } 57 \\
\mathrm{~mA} \mathrm{~h} \mathrm{~g}^{-1} \text { (cycle } \\
\text { 10). } \\
\text { b) } 60 \mathrm{~mA} \mathrm{~h} \mathrm{~g}^{-1} \\
\text { (first } \\
\text { discharge), } 255 \\
\mathrm{~mA} \mathrm{~h} \mathrm{~g}{ }^{-1} \text { (cycle } \\
\text { 10) }\end{array}$ & $\begin{array}{l}\text { Mg present in } \\
\text { discharged electrode, } \\
\text { and a small amount } \\
\text { remained after } \\
\text { charging (EDS, } \\
\text { quantification not } \\
\text { provided). }\end{array}$ & S117 \\
\hline $\mathrm{Mo}_{2.48} \mathrm{VO}_{9.93}$ & $\begin{array}{l}\text { Room } \\
\text { temperature, } \\
\text { capacitive carbon, } \\
0.5 \mathrm{M} \mathrm{Mg}(\mathrm{TFSI})_{2} \text { in } \\
\text { acetonitrile }\end{array}$ & $\begin{array}{l}\text { Expansion of the } a, b \\
\text { and c lattice } \\
\text { parameters with } \\
\text { chemical Mg insertion } \\
\text { (data for } \\
\text { electrochemically } \\
\text { discharged electrodes } \\
\text { not provided) (XRD) }\end{array}$ & Not provided & $\begin{array}{l}400 \mathrm{~mA} \mathrm{~h} \mathrm{~g}^{-1} \\
\text { (first } \\
\text { discharge), } 230 \\
\mathrm{~mA} \mathrm{~h} \mathrm{~g}{ }^{-1} \text { (cycle } \\
15 \text { ) }\end{array}$ & Not provided & S118 \\
\hline $\mathrm{Mg}_{0.1} \mathrm{~V}_{2} \mathrm{O}_{5} \cdot 2.35 \mathrm{H}_{2} \mathrm{O}$ & $\begin{array}{l}\text { Room } \\
\text { temperature, } \\
\text { carbon, } 0.5 \mathrm{M} \\
\mathrm{Mg}(\mathrm{TFSI})_{2} \text { and } 0.5 \\
\mathrm{M} \text { dipro-glyme in } \\
\text { acetonitrile }\end{array}$ & Not provided & Not provided & 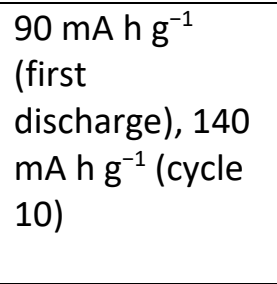 & $\begin{array}{l}\mathrm{Mg}_{0.55} \mathrm{~V}_{2} \mathrm{O}_{5} \\
\text { (discharged) } \\
\mathrm{Mg}_{0.05} \mathrm{~V}_{2} \mathrm{O}_{5} \text { (charged) } \\
\text { (ICP-AES) }\end{array}$ & S119 \\
\hline
\end{tabular}




\begin{tabular}{|c|c|c|c|c|c|c|}
\hline Material & $\begin{array}{c}\text { Cycling } \\
\text { conditions: } \\
\text { Temperature, } \\
\text { anode, and } \\
\text { electrolyte }\end{array}$ & $\begin{array}{l}\text { Magnesiated Product } \\
\text { phases }\end{array}$ & Redox changes & $\begin{array}{c}\text { Reported } \\
\text { Electrochemical } \\
\text { Capacity }\end{array}$ & $\begin{array}{c}\text { Experimentally } \\
\text { Verified Magnesiated } \\
\text { Composition }\end{array}$ & Reference \\
\hline $\mathrm{VO}_{\mathrm{x}}$ nanotubes & $\begin{array}{l}24{ }^{\circ} \mathrm{C}, \mathrm{Pt} \text { foil, } 0.5 \\
\mathrm{M} \mathrm{Mg}\left(\mathrm{ClO}_{4}\right)_{2} \text { in a) } \\
\text { acetonitrile, b) } \\
\mathrm{TMS}, \text { or } \mathrm{c}) \mathrm{TMS} \\
\text { with } 10 \text { vol\% EA }\end{array}$ & $\begin{array}{l}\text { a) Evidence of } \\
\text { electrolyte } \\
\text { decomposition (SEM). } \\
\text { c). Little or no } \\
\text { evidence of } \\
\text { electrolyte } \\
\text { decomposition (SEM) }\end{array}$ & Not provided & $\begin{array}{l}\text { a) } 220 \mathrm{~mA} \mathrm{~h} \mathrm{~g}^{-1} \\
\text { (first } \\
\text { discharge), } 65 \\
\mathrm{~mA} \mathrm{~h} \mathrm{~g}{ }^{-1} \text { (cycle } \\
60 \text { ). } \\
\text { b) } 70 \mathrm{~mA} \mathrm{~h} \mathrm{~g}^{-1} \text {, } \\
60 \mathrm{~mA} \mathrm{~h}^{-1} \\
\text { (cycle 60) c) } 120 \\
\mathrm{~mA} \mathrm{~h} \mathrm{~g}^{-1} \text { (first } \\
\text { discharge), } 90 \\
\mathrm{~mA} \mathrm{~h} \mathrm{~g} \text { (cycle } \\
\text { 60). }\end{array}$ & Not provided & S120 \\
\hline $\begin{array}{l}\mathrm{V}_{2} \mathrm{O}_{5} \cdot 1.8 \mathrm{H}_{2} \mathrm{O} \text { Xerogel } \\
\text { Material }\end{array}$ & $\begin{array}{l}\text { Room } \\
\text { temperature, } \mathrm{Mg} \\
\text { foil, } 1 \mathrm{M} \mathrm{Mg}(\mathrm{TFSI})_{2} \\
\text { in diglyme }\end{array}$ & $\begin{array}{l}\text { Consistent contraction } \\
\text { in the layer axis ( } c \\
\text { lattice parameter) } \\
\text { with discharge (X-ray } \\
\text { PDF). Mg intercalated } \\
\text { with an octahedral } \\
\text { oxygen environment } \\
\left({ }^{25} \mathrm{Mg} \text { NMR). Co- }\right. \\
\text { intercalation of Mg } \\
\text { with diglyme with } \\
\left.\text { discharge ( }{ }^{1} \mathrm{H} N \mathrm{NR}\right)\end{array}$ & $\begin{array}{l}\mathrm{V}^{5+} \text { was reduced to } \\
\mathrm{V}^{4.5+} \text { on discharge } \\
\text { (XANES) }\end{array}$ & $\begin{array}{l}22 \mathrm{~mA} \mathrm{~h} \mathrm{~g}^{-1} \\
\text { (first } \\
\text { discharge), } 45 \\
\mathrm{~mA} \mathrm{~h} \mathrm{~g}^{-1} \text { (cycle } \\
\text { 10) }\end{array}$ & Not provided & S121 \\
\hline
\end{tabular}




\begin{tabular}{|c|c|c|c|c|c|c|}
\hline Material & $\begin{array}{c}\text { Cycling } \\
\text { conditions: } \\
\text { Temperature, } \\
\text { anode, and } \\
\text { electrolyte }\end{array}$ & $\begin{array}{l}\text { Magnesiated Product } \\
\text { phases }\end{array}$ & Redox changes & $\begin{array}{c}\text { Reported } \\
\text { Electrochemical } \\
\text { Capacity }\end{array}$ & $\begin{array}{c}\text { Experimentally } \\
\text { Verified Magnesiated } \\
\text { Composition }\end{array}$ & Reference \\
\hline $\begin{array}{l}\left.\text { a) } \mathrm{V}_{2} \mathrm{O}_{5} \cdot n \mathrm{H}_{2} \mathrm{O}, \mathrm{b}\right) \\
\mathrm{V}_{2} \mathrm{O}_{5} \cdot n \mathrm{H}_{2} \mathrm{O}+5.8 \mathrm{wt} \% \\
\text { interlayer PEO, c) } \\
\mathrm{V}_{2} \mathrm{O}_{5} \cdot n \mathrm{H}_{2} \mathrm{O}+20.0 \mathrm{wt} \% \\
\text { interlayer PEO }\end{array}$ & $\begin{array}{l}\text { Room } \\
\text { temperature, } \\
\text { capacitive carbon, } \\
0.5 \mathrm{M} \mathrm{Mg}\left(\mathrm{ClO}_{4}\right)_{2} \\
\text { in acetonitrile }\end{array}$ & Not provided & Not provided & 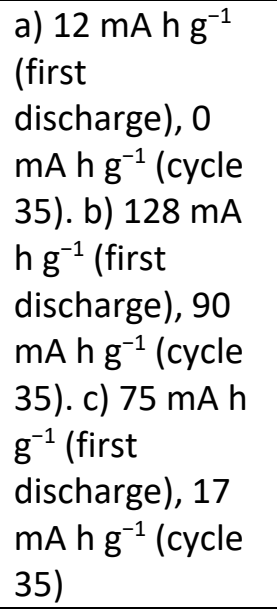 & Not provided & S122 \\
\hline$\alpha-\mathrm{V}_{2} \mathrm{O}_{5}$ & $\begin{array}{l}\text { Room } \\
\text { temperature, } \\
\text { capacitive carbon, } \\
0.5 \mathrm{M} \mathrm{Mg}\left(\mathrm{ClO}_{4}\right)_{2} \\
\text { with a) } 2 \mathrm{ppm} \mathrm{H}_{2} \mathrm{O} \\
\text { and b) } 2.0 \mathrm{M} \mathrm{H}_{2} \mathrm{O} \\
\text { in acetonitrile }\end{array}$ & $\begin{array}{l}\text { b) Unit cell expansion } \\
\text { with low depth of } \\
\text { discharge, with the } a \text { - } \\
\text { lattice parameter } \\
\text { increasing and b and } \\
\text { c lattice parameter } \\
\text { decreasing. Formation } \\
\text { of a new crystalline } \\
\text { phase with large } \\
\text { depth of discharge. } \\
\text { These changes were } \\
\text { reversed on charge } \\
\text { (XRD) }\end{array}$ & Not provided & $\begin{array}{l}\text { a) Low capacity } \\
\text { (values not } \\
\text { given) b) } 295 \\
\mathrm{~mA} \mathrm{~h}^{-1} \text { (first } \\
\text { discharge) }\end{array}$ & $\begin{array}{l}\text { b) } \mathrm{Mg}_{0.17} \mathrm{H}_{x} \mathrm{~V}_{2} \mathrm{O}_{5} \text { in } \\
\text { discharged state (EDX } \\
\text { and ICP). Quantity of } \\
\text { protons was estimated } \\
\text { in the range } 0.66 \leq x \leq \\
1.16\end{array}$ & S123 \\
\hline
\end{tabular}




\begin{tabular}{|c|c|c|c|c|c|c|}
\hline Material & $\begin{array}{c}\text { Cycling } \\
\text { conditions: } \\
\text { Temperature, } \\
\text { anode, and } \\
\text { electrolyte } \\
\end{array}$ & $\begin{array}{c}\text { Magnesiated Product } \\
\text { phases }\end{array}$ & Redox changes & $\begin{array}{c}\text { Reported } \\
\text { Electrochemical } \\
\text { Capacity }\end{array}$ & $\begin{array}{c}\text { Experimentally } \\
\text { Verified Magnesiated } \\
\text { Composition }\end{array}$ & Reference \\
\hline $\mathrm{V}_{2} \mathrm{MoO}_{8}$ & $\begin{array}{l}\left.\text { a) } 25^{\circ} \mathrm{C} \mathrm{b}\right) 50{ }^{\circ} \mathrm{C} \mathrm{c} \text { ) } \\
60{ }^{\circ} \mathrm{C}, \mathrm{Mg} \text { foil, } 0.4 \\
\mathrm{M} \mathrm{APC} \text { electrolyte } \\
\text { ( } \mathrm{PhMgCl} \text { and } \mathrm{AlCl}_{3} \\
\text { in } 2: 1 \text { molar ratio) } \\
\text { in THF }\end{array}$ & $\begin{array}{l}\text { Large irreversible } \\
\text { transformations to } \\
\mathrm{MgV}_{2} \mathrm{O}_{4} \text { and } \mathrm{MgVO}_{3} \\
\text { on discharge (XRD) }\end{array}$ & Not provided & $\begin{array}{l}\text { a) } 200 \mathrm{~mA} \mathrm{~h} \mathrm{~g}^{-1} \\
\text { (first } \\
\text { discharge), } 60 \\
\mathrm{~mA} \mathrm{~h}^{-1} \text { (cycle } \\
\text { 2). b) } 215 \mathrm{~mA} \mathrm{~h} \\
\mathrm{~g}^{-1} \text { (first } \\
\text { discharge), } 100 \\
\mathrm{~mA} \mathrm{~h}^{-1} \text { (cycle } \\
\text { 2). c) } 225 \mathrm{~mA} \mathrm{~h} \\
\mathrm{~g}^{-1} \text { (first } \\
\text { discharge), } 135 \\
\mathrm{~mA} \mathrm{~h} \mathrm{~g}^{-1} \text { (cycle } \\
\text { 2). }\end{array}$ & Not provided & S124 \\
\hline$\alpha-\mathrm{V}_{2} \mathrm{O}_{5}$ & $\begin{array}{l}\text { Room } \\
\text { temperature, } \mathrm{Mg} \\
\text { foil, } 1.0 \mathrm{M} \\
\mathrm{Mg}(\mathrm{TFSI})_{2} \text { in } \\
\text { diglyme }(<20 \mathrm{ppm} \\
\left.\mathrm{H}_{2} \mathrm{O}\right)\end{array}$ & $\begin{array}{l}\text { Partial formation of } \\
\text { the } \varepsilon-\mathrm{Mg}_{0.5} \mathrm{~V}_{2} \mathrm{O}_{5} \text { phase } \\
\text { on discharge (HAADF- } \\
\text { STEM) }\end{array}$ & Not provided & $\begin{array}{l}\text { a) } 10 \mathrm{~mA} \mathrm{~h} \mathrm{~g}^{-1} \\
\text { (first } \\
\text { discharge), } 33 \\
\mathrm{~mA} \mathrm{~h} \mathrm{g-1} \text { (cycle } \\
\text { 6) }\end{array}$ & $\mathrm{Mg}_{0.17} \mathrm{~V}_{2} \mathrm{O}_{5}(\mathrm{EDX})$ & S125 \\
\hline $\mathrm{H}_{2} \mathrm{~V}_{3} \mathrm{O}_{8}$ & $\begin{array}{l}\text { Room } \\
\text { temperature, } \\
\text { capacitive carbon, } \\
0.5 \mathrm{M} \mathrm{Mg}\left(\mathrm{ClO}_{4}\right)_{2} \\
\text { in acetonitrile }\end{array}$ & Not provided & Not provided & $\begin{array}{l}305 \mathrm{~mA} \mathrm{~h} \mathrm{~g}^{-1} \\
\text { (first } \\
\text { discharge), } 250 \\
\mathrm{~mA} \mathrm{~h} \mathrm{~g}{ }^{-1} \text { (cycle } \\
\text { 20) }\end{array}$ & Not provided & S126 \\
\hline
\end{tabular}




\begin{tabular}{|c|c|c|c|c|c|c|}
\hline Material & $\begin{array}{c}\text { Cycling } \\
\text { conditions: } \\
\text { Temperature, } \\
\text { anode, and } \\
\text { electrolyte }\end{array}$ & $\begin{array}{c}\text { Magnesiated Product } \\
\text { phases }\end{array}$ & Redox changes & $\begin{array}{c}\text { Reported } \\
\text { Electrochemical } \\
\text { Capacity }\end{array}$ & $\begin{array}{c}\text { Experimentally } \\
\text { Verified Magnesiated } \\
\text { Composition }\end{array}$ & Reference \\
\hline $\begin{array}{l}\left(\mathrm{C}_{12} \mathrm{H}_{25} \mathrm{NH}_{3}\right)_{0.24} \mathrm{VO}_{x} \\
\text { nanotubes }\end{array}$ & $\begin{array}{l}\text { Room } \\
\text { temperature, } \mathrm{Mg} \\
\text { foil, a) } 1 \mathrm{M} \\
\mathrm{Mg}(\mathrm{TFSI})_{2} \text { in } \\
\text { diglyme b) } 1 \mathrm{M} \\
\mathrm{Mg}\left(\mathrm{ClO}_{4}\right)_{2} \text { in } \\
\text { acetonitrile }\end{array}$ & $\begin{array}{l}\text { b) Expansion of } a, b \\
\text { and } c \text { lattice } \\
\text { parameters with } \\
\text { discharge (XRD) }\end{array}$ & $\begin{array}{l}\text { Reduction of } \mathrm{V}^{4+} \text { to } \\
\mathrm{V}^{3+} \text { suggested by } \\
\text { change in } \\
\text { coordination } \\
\text { geometries (X-ray } \\
\text { PDF) }\end{array}$ & $\begin{array}{l}\text { a) } 72 \pm 5 \mathrm{~mA} \mathrm{~h} \\
\mathrm{~g}^{-1} \text { (first } \\
\text { discharge, } \\
\text { averaged over } 3 \\
\text { cells), } 35 \mathrm{~mA} \mathrm{~g}^{-1} \\
\text { (cycle 10) b) } \\
146 \pm 35 \mathrm{~mA} \mathrm{~h} \\
\mathrm{~g}^{-1} \text { (first } \\
\text { discharge, } \\
\text { averaged over } 3 \\
\text { cells), } 25 \mathrm{~mA} \mathrm{~g}^{-1} \\
\text { (cycle 10) }\end{array}$ & $\begin{array}{l}\text { a) } \mathrm{Mg}_{1.75} \mathrm{~V}_{7} \mathrm{O}_{16} \\
\text { (discharged), } \\
\mathrm{Mg}_{0.5} \mathrm{~V}_{7} \mathrm{O}_{16} \text { (charged) } \\
\text { b) } \mathrm{Mg}_{5.5} \mathrm{~V}_{7} \mathrm{O}_{16} \\
\text { (discharged), } \\
\mathrm{Mg}_{2.75} \mathrm{~V}_{7} \mathrm{O}_{16} \text { (charged) }\end{array}$ & S127 \\
\hline $\begin{array}{l}\mathrm{Mg}_{0.07}\left(\mathrm{C}_{12} \mathrm{H}_{25} \mathrm{NH}_{3}\right)_{0.13} \mathrm{VO}_{\mathrm{x}} \\
\text { nanotubes }\end{array}$ & $\begin{array}{l}\text { Room } \\
\text { temperature, } \mathrm{Mg} \\
\text { foil, a) } 1 \mathrm{M} \\
\mathrm{Mg}(\mathrm{TFSI})_{2} \text { in } \\
\text { diglyme b) } 1 \mathrm{M} \\
\mathrm{Mg}\left(\mathrm{ClO}_{4}\right)_{2} \text { in } \\
\text { acetonitrile }\end{array}$ & $\begin{array}{l}\text { b) Expansion of } a, b \\
\text { and contraction of } c \\
\text { lattice parameters } \\
\text { with discharge (XRD) }\end{array}$ & $\begin{array}{l}\text { Reduction of } \mathrm{V}^{4+} \text { to } \\
\mathrm{V}^{3+} \text { suggested by } \\
\text { change in } \\
\text { coordination } \\
\text { geometries (X-ray } \\
\text { PDF) }\end{array}$ & $\begin{array}{l}\text { a) } 33 \pm 12 \mathrm{~mA} \mathrm{~h} \\
\mathrm{~g}^{-1} \text { (first } \\
\text { discharge, } \\
\text { averaged over } 3 \\
\text { cells), } 25 \mathrm{~mA} \mathrm{~g}^{-1} \\
\text { (cycle 10) b). } 58 \\
\pm 8 \mathrm{~mA} \mathrm{~h} \mathrm{~g}^{-1} \\
\text { (first discharge, } \\
\text { averaged over } 3 \\
\text { cells), } 0 \mathrm{~mA} \mathrm{~h} \\
\mathrm{~g}^{-1} \text { (cycle } 10 \text { ) }\end{array}$ & $\begin{array}{l}\text { a) } \mathrm{Mg}_{1.0} \mathrm{~V}_{7} \mathrm{O}_{16} \\
\text { (discharged), } \\
\mathrm{Mg}_{0.8} \mathrm{~V}_{7} \mathrm{O}_{16} \text { (charged) } \\
\text { b) } \mathrm{Mg}_{1.8} \mathrm{~V}_{7} \mathrm{O}_{16} \\
\text { (discharged), } \\
\mathrm{Mg}_{1.7} \mathrm{~V}_{7} \mathrm{O}_{16} \text { (charged) }\end{array}$ & S127 \\
\hline $\mathrm{NaV}_{3} \mathrm{O}_{8} \cdot 1.69 \mathrm{H}_{2} \mathrm{O}$ & $\begin{array}{l}25^{\circ} \mathrm{C}, \mathrm{Mg} \text { foil, } 0.4 \\
\mathrm{M} \mathrm{APC} \text { electrolyte } \\
\text { ( } \mathrm{PhMgCl} \text { and } \mathrm{AlCl}_{3} \\
\text { in } 2: 1 \text { molar ratio) } \\
\text { in THF }\end{array}$ & $\begin{array}{l}\text { Contraction of unit } \\
\text { cell with discharge, } \\
\text { was not reversed on } \\
\text { charge. (XRD) }\end{array}$ & Not provided & $\begin{array}{l}61 \mathrm{~mA} \mathrm{~h} \mathrm{~g}^{-1} \\
\text { (first } \\
\text { discharge), } 38 \\
\mathrm{~mA} \mathrm{~h} \mathrm{~g}^{-1} \text { (cycle } \\
100) .\end{array}$ & $\begin{array}{l}\text { Mg entrapment in } \\
\text { samples with cycling, } \\
\text { stoichiometric ratios } \\
\text { not given (ICP-MS) }\end{array}$ & S128 \\
\hline
\end{tabular}




\begin{tabular}{|c|c|c|c|c|c|c|}
\hline Material & $\begin{array}{c}\text { Cycling } \\
\text { conditions: } \\
\text { Temperature, } \\
\text { anode, and } \\
\text { electrolyte }\end{array}$ & $\begin{array}{c}\text { Magnesiated Product } \\
\text { phases }\end{array}$ & Redox changes & $\begin{array}{c}\text { Reported } \\
\text { Electrochemical } \\
\text { Capacity }\end{array}$ & $\begin{array}{c}\text { Experimentally } \\
\text { Verified Magnesiated } \\
\text { Composition }\end{array}$ & Reference \\
\hline $\mathrm{MgV}_{2} \mathrm{O}_{6}$ & $\begin{array}{l}\text { Room } \\
\text { temperature, } \mathrm{Mg} \\
\text { foil, } 1 \mathrm{M} \\
\mathrm{Mg}\left(\mathrm{ClO}_{4}\right)_{2} \text { in } \\
\text { acetonitrile }\end{array}$ & Not provided & Not provided & $\begin{array}{l}120 \mathrm{~mA} \mathrm{~h} \mathrm{~g}^{-1} \\
\text { (first } \\
\text { discharge), } 40 \\
\mathrm{~mA} \mathrm{~h} \mathrm{~g}^{-1} \text { (cycle } \\
\text { 10) }\end{array}$ & Not provided & S129 \\
\hline $\begin{array}{l}\text { a) } \mathrm{VOPO}_{4} \cdot 2 \mathrm{H}_{2} \mathrm{O}, \text { b) } \\
\mathrm{VOPO}_{4} \cdot \mathrm{xH}_{2} \mathrm{O} \cdot \mathrm{PA}\end{array}$ & $\begin{array}{l}25^{\circ} \mathrm{C}, \mathrm{Mg} \text { foil, } \\
0.25 \mathrm{M} \text { APC } \\
\text { electrolyte } \\
\text { (PhMgCl and } \mathrm{AlCl}_{3} \\
\text { in } 2: 1 \text { molar ratio) } \\
\text { in THF }\end{array}$ & $\begin{array}{l}\text { Contraction of layer } \\
\text { axis ( } c \text { lattice } \\
\text { parameter) on } \\
\text { discharge. Expansion } \\
\text { to original position } \\
\text { observed with charge. } \\
\text { (XRD) }\end{array}$ & $\begin{array}{l}\text { Surface vanadium } \\
\text { was reduced on } \\
\text { discharge, and } \\
\text { oxidized on charge } \\
\text { (XPS) }\end{array}$ & $\begin{array}{l}\text { a) } 280 \mathrm{~mA} \mathrm{~h} \mathrm{~g}^{-1} \\
\text { (first } \\
\text { discharge), } 120 \\
\mathrm{~mA} \mathrm{~h} \mathrm{~g}^{-1} \text { (cycle } \\
\text { 140) b) } 280 \mathrm{~mA} \\
\mathrm{~h} \mathrm{~g}^{-1} \text { (first } \\
\text { discharge), } 192 \\
\mathrm{~mA} \mathrm{~h} \mathrm{~g}^{-1} \text { (cycle } \\
500 \text { ). }\end{array}$ & $\begin{array}{l}\text { a) Sole incorporation } \\
\text { of } \mathrm{Mg} \text {. b) 1:1 co- } \\
\text { incorporation of } \mathrm{Mg} \\
\text { and } \mathrm{Cl} \text { on discharge. } \\
\text { Absolute } \\
\text { quantification of } \mathrm{Mg} \\
\text { w.r.t } \mathrm{V} \text { not given. } \\
\text { Limited removal of } \mathrm{Mg} \\
\text { and } \mathrm{Cl} \text { on charge (as } \\
\text { opposed to } \mathrm{MgCl}{ }^{+} \text {). } \\
\text { (ICP-AES, EDS, XPS, } \\
\text { weighing electrodes) }\end{array}$ & S130 \\
\hline
\end{tabular}




\begin{tabular}{|c|c|c|c|c|c|c|}
\hline Material & $\begin{array}{c}\text { Cycling } \\
\text { conditions: } \\
\text { Temperature, } \\
\text { anode, and } \\
\text { electrolyte }\end{array}$ & $\begin{array}{l}\text { Magnesiated Product } \\
\text { phases }\end{array}$ & Redox changes & $\begin{array}{c}\text { Reported } \\
\text { Electrochemical } \\
\text { Capacity }\end{array}$ & $\begin{array}{c}\text { Experimentally } \\
\text { Verified Magnesiated } \\
\text { Composition }\end{array}$ & Reference \\
\hline $\mathrm{NaV}_{2}\left(\mathrm{PO}_{4}\right)_{3} / \mathrm{C}$ & $\begin{array}{l}\text { Room } \\
\text { temperature, } \\
\text { capacitive carbon, } \\
0.1 \mathrm{M} \mathrm{Mg}(\mathrm{TFSI})_{2} \text { in } \\
\text { acetonitrile (< } \\
0.002 \% \text { water) }\end{array}$ & $\begin{array}{l}\text { Expansion of the unit } \\
\text { cell with discharge, } \\
\text { yielding two phases } \\
\text { with different degrees } \\
\text { of expansion at partial } \\
\text { discharge. Full } \\
\text { discharge yielded a } \\
\text { single phase with } \\
\text { expanded lattice } \\
\text { parameters. The cell } \\
\text { contracted back to the } \\
\text { pristine with charge, } \\
\text { with only one phase } \\
\text { observed. (XRD) }\end{array}$ & $\begin{array}{l}\text { Surface } \mathrm{V}^{3.5+} \text { was } \\
\text { reduced to } \mathrm{V}^{3.1+} \text { on } \\
\text { discharge (XPS). } \\
\text { Evidence of bulk } \\
\text { reduction on } \\
\text { discharge, not } \\
\text { quantified (EPR) }\end{array}$ & $\begin{array}{l}100 \mathrm{~mA} \mathrm{~h} \mathrm{~g}^{-1} \\
\text { (maximum } \\
\text { discharge } \\
\text { capacity) }\end{array}$ & Not provided & S131 \\
\hline $\mathrm{VOPO}_{4}$ & $\begin{array}{l}\text { Room } \\
\text { temperature, } \\
\text { capacitive carbon, } \\
0.1 \mathrm{M} \text { of } \\
\mathrm{Mg}\left(\mathrm{ClO}_{4}\right)_{2} \text { in } \mathrm{PC} \\
\text { with a) no water } \\
\text { added or b) } 0.6 \mathrm{M} \\
\text { water added }\end{array}$ & Not provided & Not provided & $\begin{array}{l}\text { a) } 8 \mathrm{~mA} \mathrm{~h} \mathrm{~g}^{-1} \\
\text { (first } \\
\text { discharge). b) } \\
11 \mathrm{~mA} \mathrm{~h} \mathrm{~g}^{-1} \\
\text { (first discharge) }\end{array}$ & Not provided & S132 \\
\hline
\end{tabular}




\begin{tabular}{|c|c|c|c|c|c|c|}
\hline Material & $\begin{array}{c}\text { Cycling } \\
\text { conditions: } \\
\text { Temperature, } \\
\text { anode, and } \\
\text { electrolyte }\end{array}$ & $\begin{array}{c}\text { Magnesiated Product } \\
\text { phases }\end{array}$ & Redox changes & $\begin{array}{l}\text { Reported } \\
\text { Electrochemical } \\
\text { Capacity }\end{array}$ & $\begin{array}{c}\text { Experimentally } \\
\text { Verified Magnesiated } \\
\text { Composition }\end{array}$ & Reference \\
\hline $\mathrm{VOPO}_{4} \cdot 2 \mathrm{H}_{2} \mathrm{O}$ & $\begin{array}{l}\text { Room } \\
\text { temperature, } \\
\text { capacitive carbon, } \\
0.1 \mathrm{M} \text { of } \\
\mathrm{Mg}\left(\mathrm{ClO}_{4}\right)_{2} \text { in } \mathrm{PC} \\
\text { with a) no water } \\
\text { added or b) } 0.6 \mathrm{M} \\
\text { water added }\end{array}$ & $\begin{array}{l}\text { a) Contraction of layer } \\
\text { axis (c-lattice } \\
\text { parameter) and large } \\
\text { reduction of } \\
\text { crystallinity with } \\
\text { discharge. Partial re- } \\
\text { expansion of the c- } \\
\text { lattice parameter with } \\
\text { charge (does not } \\
\text { return to original } \\
\text { pristine value).b) } \\
\text { Contraction of layer } \\
\text { axis (c-lattice } \\
\text { parameter) with } \\
\text { discharge. Crystallinity } \\
\text { is retained. Partial re- } \\
\text { expansion of the c- } \\
\text { lattice parameter with } \\
\text { charge (does not } \\
\text { return to original } \\
\text { pristine value). }\end{array}$ & Not provided & $\begin{array}{l}\text { a) } 14 \mathrm{~mA} \mathrm{~h} \mathrm{~g}^{-1} \\
\text { (first discharge) } \\
\text { b) } 92 \mathrm{~mA} \mathrm{~h} \mathrm{~g}^{-1} \\
\text { (first discharge) }\end{array}$ & Not provided & S132 \\
\hline
\end{tabular}




\begin{tabular}{|c|c|c|c|c|c|c|}
\hline Material & $\begin{array}{c}\text { Cycling } \\
\text { conditions: } \\
\text { Temperature, } \\
\text { anode, and } \\
\text { electrolyte }\end{array}$ & $\begin{array}{l}\text { Magnesiated Product } \\
\text { phases }\end{array}$ & Redox changes & $\begin{array}{c}\text { Reported } \\
\text { Electrochemical } \\
\text { Capacity }\end{array}$ & $\begin{array}{c}\text { Experimentally } \\
\text { Verified Magnesiated } \\
\text { Composition }\end{array}$ & Reference \\
\hline$\alpha-\mathrm{V}_{2} \mathrm{O}_{5}$ & $\begin{array}{l}\text { Room } \\
\text { temperature, } \\
\text { capacitive carbon, } \\
\text { a) } 0.25 \mathrm{M} \\
\mathrm{Mg}\left(\mathrm{ClO}_{4}\right)_{2} \text { in } \\
\text { acetonitrile, b) } \\
0.25 \mathrm{M} \mathrm{Mg}\left(\mathrm{ClO}_{4}\right)_{2} \\
\text { in } \\
\text { acetonitrile:DME } \\
\text { (1:1 vol ratio) }\end{array}$ & Not provided & Not provided & $\begin{array}{l}\text { a) } 165 \mathrm{~mA} \mathrm{~h} \mathrm{~g}^{-1} \\
\text { (first discharge) } \\
\text { b) } 115 \mathrm{~mA} \mathrm{~h} \mathrm{~g}^{-1} \\
\text { (first discharge) }\end{array}$ & Not provided & S133 \\
\hline$\zeta-\mathrm{V}_{2} \mathrm{O}_{5}$ & $\begin{array}{l}50{ }^{\circ} \mathrm{C} \text {, capacitive } \\
\text { carbon, } 0.2 \mathrm{M} \\
\mathrm{Mg}(\mathrm{TFSI})_{2} \text { in } \mathrm{PC}\end{array}$ & $\begin{array}{l}\text { Discharge/charge } \\
\text { gave partially } \\
\text { reversible lattice } \\
\text { expansion/contraction } \\
\text { (XRD) }\end{array}$ & $\begin{array}{l}\text { Evidence for } \mathrm{V}^{5+} \\
\text { reduction to } \mathrm{V}^{4+} \\
\text { with discharge } \\
\text { (EELS) }\end{array}$ & 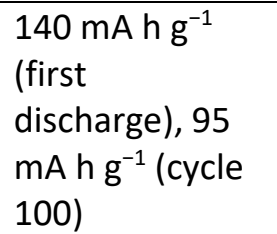 & $\begin{array}{l}\mathrm{Mg}_{0.26} \mathrm{~V}_{2} \mathrm{O}_{5}-\mathrm{Mg}_{0.32} \mathrm{~V}_{2} \mathrm{O}_{5} \\
\text { in the discharged state } \\
\text { (EDX line scan) }\end{array}$ & S134 \\
\hline $\mathrm{VO}_{2}(\mathrm{~B})$ & $\begin{array}{l}20{ }^{\circ} \mathrm{C} \text {, carbon, } 1 \mathrm{M} \\
\mathrm{Mg}\left(\mathrm{ClO}_{4}\right)_{2} \text { in } \\
\text { acetonitrile }\end{array}$ & $\begin{array}{l}\text { Partial loss of } \\
\text { crystallinity on } \\
\text { discharge, which is } \\
\text { restored on charge. } \\
\text { No other obvious } \\
\text { crystallographic } \\
\text { changes (XRD) }\end{array}$ & $\begin{array}{l}\text { Reduction of } \\
\text { surface } \mathrm{V}^{4.1+} \text { to } \mathrm{V}^{3.0+} \\
\text { on discharge, } \\
\text { oxidation to } \mathrm{V}^{3.9+} \text { on } \\
\text { charge (XPS). } \\
\text { Surface } \mathrm{Mg} \text { evident } \\
\text { on discharge, not } \\
\text { evident on charge } \\
\text { (XPS). }\end{array}$ & $\begin{array}{l}400 \mathrm{~mA} \mathrm{~h} \mathrm{~g}^{-1} \\
\text { (first } \\
\text { discharge), } 200 \\
\mathrm{~mA} \mathrm{~h} \mathrm{~g}^{-1} \text { (cycle } \\
60 \text { ) }\end{array}$ & Not provided & S135 \\
\hline$\alpha-V_{2} O_{5}$ (thin film) & $\begin{array}{l}\text { Room } \\
\text { temperature, } \mathrm{Pt} \\
\text { foil, } 0.075 \mathrm{M} \\
\mathrm{MgCl}_{2} \text { in water }\end{array}$ & $\begin{array}{l}\text { Expansion of unit cell } \\
\text { with discharge (XRD). }\end{array}$ & Not provided & $\begin{array}{l}427 \mathrm{~mA} \mathrm{~h} \mathrm{~g}^{-1} \\
\text { (first } \\
\text { discharge), } 350 \\
\mathrm{~mA} \mathrm{~h} \mathrm{~g}^{-1} \text { (cycle } \\
2000 \text { ) }\end{array}$ & $\begin{array}{l}\sim \mathrm{Mg}_{40} \mathrm{~V}_{2} \mathrm{O}_{5} \text { (XPS depth } \\
\text { profiling) }\end{array}$ & S136 \\
\hline
\end{tabular}




\begin{tabular}{|c|c|c|c|c|c|c|}
\hline Material & $\begin{array}{c}\text { Cycling } \\
\text { conditions: } \\
\text { Temperature, } \\
\text { anode, and } \\
\text { electrolyte }\end{array}$ & $\begin{array}{c}\text { Magnesiated Product } \\
\text { phases }\end{array}$ & Redox changes & $\begin{array}{c}\text { Reported } \\
\text { Electrochemical } \\
\text { Capacity }\end{array}$ & $\begin{array}{c}\text { Experimentally } \\
\text { Verified Magnesiated } \\
\text { Composition }\end{array}$ & Reference \\
\hline$\alpha-V_{2} O_{5}$ & $\begin{array}{l}100{ }^{\circ} \mathrm{C}, \mathrm{Mg} \text { foil, a) } \\
0.3 \mathrm{M} \mathrm{Mg}(\mathrm{TFSI})_{2} \text { in } \\
\text { 1:1 volume ratio } \\
\text { of EC:PC with <25 } \\
\mathrm{ppm} \text { water. b) } 0.3 \\
\mathrm{M} \mathrm{Mg}(\mathrm{TFSI})_{2} \text { in } 1: 1 \\
\text { volume ratio of } \\
\mathrm{EC}: \mathrm{PC} \text { with } \\
>15000 \mathrm{ppm} \\
\text { water. c) } 0.3 \mathrm{M} \\
\mathrm{Mg}\left(\mathrm{ClO}_{4}\right)_{2} \text { in } 1: 1 \\
\text { volume ratio of } \\
\text { EC:PC with } \\
>15000 \text { ppm } \\
\text { water. }\end{array}$ & $\begin{array}{l}\text { a-c) Formation of } \\
\text { various protonated } \\
\text { phases during } \\
\text { discharge, culminating } \\
\text { in Häggite }\left(\mathrm{H}_{4} \mathrm{~V}_{4} \mathrm{O}_{10}\right) \text { at } \\
\text { the full depth of } \\
\text { discharge. Occupation } \\
\text { of } \mathrm{Mg} \text { in the lattice. } \\
\text { was either zero or } \\
\text { very little. } \\
\text { Reformation of } \alpha-\mathrm{V}_{2} \mathrm{O}_{5} \\
\text { on recharge. } \\
\text { Formation of } \\
\text { protonated phases } \\
\text { was favored by water } \\
\text { inclusion, but even } \\
\text { occurred in dry } \\
\text { electrolytes. } \\
\text { Formation of } \\
\text { protonated phases } \\
\text { even occurred at } \\
\text { elevated } \\
\text { temperatures in the } \\
\text { Mg electrolytes with } \\
\text { no current or voltage } \\
\text { applied. (XRD and } \\
\text { electron diffraction) }\end{array}$ & Not provided & $\begin{array}{l}\text { a-b) Not } \\
\text { provided, c) } \\
530 \mathrm{~mA} \mathrm{~h} \mathrm{~g}^{-1} \\
\text { (first discharge) }\end{array}$ & Not provided & S137 \\
\hline
\end{tabular}




\begin{tabular}{|c|c|c|c|c|c|c|}
\hline Material & $\begin{array}{c}\text { Cycling } \\
\text { conditions: } \\
\text { Temperature, } \\
\text { anode, and } \\
\text { electrolyte }\end{array}$ & $\begin{array}{c}\text { Magnesiated Product } \\
\text { phases }\end{array}$ & Redox changes & $\begin{array}{c}\text { Reported } \\
\text { Electrochemical } \\
\text { Capacity }\end{array}$ & $\begin{array}{c}\text { Experimentally } \\
\text { Verified Magnesiated } \\
\text { Composition }\end{array}$ & Reference \\
\hline$\alpha-V_{2} O_{5}$ & $\begin{array}{l}110^{\circ} \mathrm{C} \text {, capacitive } \\
\text { carbon, } 0.5 \mathrm{M} \\
\mathrm{Mg}(\mathrm{TFSI})_{2} \text { in } \\
\mathrm{PY}_{14} \mathrm{TFSI}(43.7 \\
\text { ppm water) }\end{array}$ & $\begin{array}{l}\text { Complex phase } \\
\text { changes of } \mathrm{V}_{2} \mathrm{O}_{5} \\
\text { occurred during } \\
\text { discharge. The newly } \\
\text { formed phases did not } \\
\text { correspond to any } \\
\text { known protonated or } \\
\mathrm{Mg} \text {-containing } \mathrm{V}_{2} \mathrm{O}_{5} \\
\text { polymorphs. Charging } \\
\text { recovered the } \alpha-\mathrm{V}_{2} \mathrm{O}_{5} \\
\text { lattice. Discharge in } \\
\text { pure } \mathrm{PY}_{14} \mathrm{TFSI} \text { yielded } \\
\text { different products } \\
\text { alongside the pristine } \\
\mathrm{V}_{2} \mathrm{O}_{5} \text {. (XRD) Structural } \\
\text { distortion and a lack } \\
\text { of protons detected in } \\
\text { the } \mathrm{V}_{2} \mathrm{O}_{5} \text { lattice on } \\
\text { discharge (FTIR). } \\
\text { Structural } \\
\text { delamination of } \\
\text { particles during } \\
\text { discharge (STEM). }\end{array}$ & $\begin{array}{l}\text { Signature of } \mathrm{MgO} \\
\text { was absent. } \\
\text { Reduction of } \mathrm{V}^{5+} \text { to } \\
\mathrm{V}^{4+} \text { on discharge } \\
\text { (bulk), reduction to } \\
\mathrm{V}^{3+} \text { at the surface. } \\
\text { Changes were } \\
\text { largely reversed on } \\
\text { charge (XAS). } \\
\text { Smaller particles } \\
\text { demonstrated } \\
\text { greater redox } \\
\text { activity, and } \\
\text { enhanced reactivity } \\
\text { of the surface was } \\
\text { observed (STXM) }\end{array}$ & $\begin{array}{l}295 \mathrm{~mA} \mathrm{~h} \mathrm{~g}^{-1} \\
\text { (first } \\
\text { discharge), } 200 \\
\mathrm{~mA} \mathrm{~h} \mathrm{~g}{ }^{-1} \text { (cycle } \\
50 \text { ) }\end{array}$ & $\begin{array}{l}\mathrm{Mg}_{1.1 .} \mathrm{V}_{2} \mathrm{O}_{5} \\
\text { (discharged), } \\
\mathrm{Mg}_{0.2} \mathrm{~V}_{2} \mathrm{O}_{5} \text { (charged). } \\
\text { Small quantity of } \mathrm{S} \\
\text { and } \mathrm{N} \text { (discharged) } \\
\text { suggested co- } \\
\text { intercalation of TFSI } \\
\text { was insignificant. } \\
\text { (EDS). }\end{array}$ & S138 \\
\hline
\end{tabular}




\begin{tabular}{|c|c|c|c|c|c|c|}
\hline Material & $\begin{array}{c}\text { Cycling } \\
\text { conditions: } \\
\text { Temperature, } \\
\text { anode, and } \\
\text { electrolyte }\end{array}$ & $\begin{array}{l}\text { Magnesiated Product } \\
\text { phases }\end{array}$ & Redox changes & $\begin{array}{c}\text { Reported } \\
\text { Electrochemical } \\
\text { Capacity }\end{array}$ & $\begin{array}{c}\text { Experimentally } \\
\text { Verified Magnesiated } \\
\text { Composition }\end{array}$ & Reference \\
\hline$\beta-\mathrm{NaV}_{6} \mathrm{O}_{15}$ & $\begin{array}{l}\text { Room } \\
\text { temperature, } \\
\text { capacitive carbon, } \\
0.5 \mathrm{M} \mathrm{Mg}\left(\mathrm{ClO}_{4}\right)_{2} \\
\text { in acetonitrile } \\
\text { (anhydrous) }\end{array}$ & $\begin{array}{l}\text { Contraction of the c- } \\
\text { axis on low depth of } \\
\text { discharge ( } 75 \mathrm{~mA} \mathrm{~h} \\
\mathrm{~g}^{-1} \text { ), followed by } \\
\text { expansion at deeper } \\
\text { discharge (from } 75 \\
\mathrm{~mA} \mathrm{~h} \mathrm{~g}^{-1} \text { to } 215 \mathrm{~mA} \mathrm{~h} \\
\left.\mathrm{~g}^{-1}\right) . \text { The changes } \\
\text { were reversed on } \\
\text { charge. (XRD, TEM) }\end{array}$ & $\begin{array}{l}\text { Surface } \mathrm{V}^{5+} \text { is } \\
\text { reduced to } \mathrm{V}^{4.5+} \text { on } \\
\text { discharge to } 215 \\
\mathrm{~mA} \mathrm{~h}^{-1} \text {, and is } \\
\text { reversed on charge } \\
\text { (XPS) }\end{array}$ & $\begin{array}{l}215 \mathrm{~mA} \mathrm{~h} \mathrm{~g}^{-1} \\
\text { (first } \\
\text { discharge), } 225 \\
\mathrm{~mA} \mathrm{~h} \mathrm{~g}^{-1} \text { (cycle } \\
\text { 5) }\end{array}$ & Not provided & S139 \\
\hline $\begin{array}{l}\left.\text { a) } \mathrm{LiV}_{3} \mathrm{O}_{8}, \text { b) } \mathrm{NaV}_{3} \mathrm{O}_{8}, \mathrm{c}\right) \\
\mathrm{KV}_{3} \mathrm{O}_{8}\end{array}$ & $\begin{array}{l}\text { Room } \\
\text { temperature, } \mathrm{Mg} \\
\text { foil, } 0.5 \mathrm{M} \\
\mathrm{Mg}\left(\mathrm{ClO}_{4}\right)_{2} \text { in } \\
\text { acetonitrile }\end{array}$ & 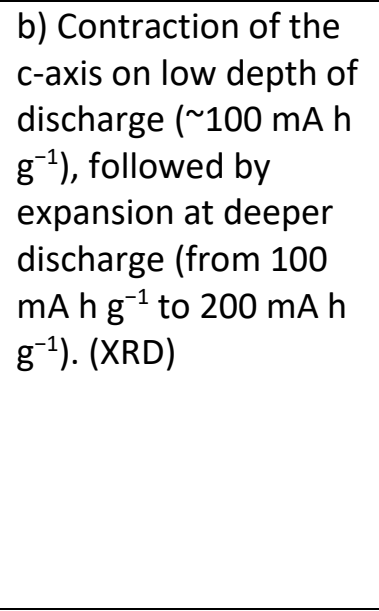 & $\begin{array}{l}\text { b) Evidence of } \\
\text { reversible surface } \\
\mathrm{V}^{5+} / \mathrm{V}^{4+} \text { redox } \\
\text { activity with } \\
\text { discharge and } \\
\text { charge (not } \\
\text { quantified) (XPS) }\end{array}$ & 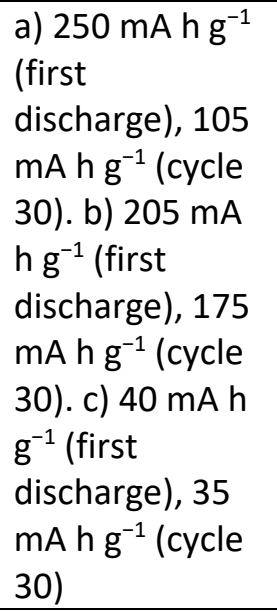 & $\begin{array}{l}\text { b) Uniform dispersion } \\
\text { of Mg found in } \\
\text { discharged particle } \\
\text { (not quantified) } \\
\text { (HAADF-EDS) }\end{array}$ & S140 \\
\hline$\alpha-\mathrm{V}_{2} \mathrm{O}_{5}$ & $\begin{array}{l}\text { Room } \\
\text { temperature, } \\
\text { capacitive carbon, } \\
0.2 \mathrm{M} \mathrm{Mg}(\mathrm{TFSI})_{2} \text { in } \\
\text { acetonitrile }(<21 \\
\text { ppm water })\end{array}$ & Not provided & Not provided & Not provided & $\begin{array}{l}\text { Evidence of } \mathrm{MgF}_{2} \\
\text { decomposition } \\
\text { product on the } \mathrm{V}_{2} \mathrm{O}_{5} \\
\text { electrode surface } \\
\text { (XPS) }\end{array}$ & S141 \\
\hline
\end{tabular}




\begin{tabular}{|c|c|c|c|c|c|c|}
\hline Material & $\begin{array}{c}\text { Cycling } \\
\text { conditions: } \\
\text { Temperature, } \\
\text { anode, and } \\
\text { electrolyte }\end{array}$ & $\begin{array}{l}\text { Magnesiated Product } \\
\text { phases }\end{array}$ & Redox changes & $\begin{array}{c}\text { Reported } \\
\text { Electrochemical } \\
\text { Capacity }\end{array}$ & $\begin{array}{c}\text { Experimentally } \\
\text { Verified Magnesiated } \\
\text { Composition }\end{array}$ & Reference \\
\hline $\begin{array}{l}\left.\text { a) } \mathrm{Mg}_{0.3} \mathrm{~V}_{2} \mathrm{O}_{5}, \mathrm{~b}\right) \\
\mathrm{Mg}_{0.3} \mathrm{~V}_{2} \mathrm{O}_{5} \cdot 1.1 \mathrm{H}_{2} \mathrm{O}\end{array}$ & $\begin{array}{l}\text { Room } \\
\text { temperature, } \\
\text { capacitive carbon, } \\
0.3 \mathrm{M} \mathrm{Mg}(\mathrm{TFSI})_{2} \text { in } \\
\text { acetonitrile }\end{array}$ & $\begin{array}{l}\text { b) Reduced } \\
\text { crystallinity and layer } \\
\text { axis contraction (c- } \\
\text { lattice parameter) } \\
\text { with discharge. These } \\
\text { changes were } \\
\text { reversed on charge. } \\
\text { (XRD) }\end{array}$ & $\begin{array}{l}\text { b) Evidence of } \\
\text { reduction of } \mathrm{V}^{5+} \text { to } \\
\mathrm{V}^{4+} \text { on discharge } \\
\text { (XANES) }\end{array}$ & $\begin{array}{l}\text { a) } 95 \mathrm{~mA} \mathrm{~h} \mathrm{~g}^{-1} \\
\text { (first cycle), } 10 \\
\mathrm{~mA} \mathrm{~h} \mathrm{~g} \text { (cycle } \\
500) . \text { b) } 165 \mathrm{~mA} \\
\mathrm{~h} \mathrm{~g}^{-1} \text { (first } \\
\text { discharge), } 170 \\
\mathrm{~mA} \mathrm{~h} \mathrm{~g} \text { (cycle } \\
500) .\end{array}$ & $\begin{array}{l}\text { b) Evidence for lattice } \\
\mathrm{Mg} \text {, which increases } \\
\text { on discharge, and } \\
\text { decreases on charge } \\
\left({ }^{25} \mathrm{Mg} \mathrm{NMR}\right) \text {. } \\
\mathrm{Mg}_{0.56} \mathrm{~V}_{2} \mathrm{O}_{5} \cdot \mathrm{nH}_{2} \mathrm{O} \\
\text { (discharged), } \\
\mathrm{Mg}_{0.17} \mathrm{~V}_{2} \mathrm{O}_{5} \cdot \mathrm{nH}_{2} \mathrm{O} \\
\text { (charged) (EDS). Water } \\
\text { molecules coordinate } \\
\text { to lattice } \mathrm{Mg} \text {, but the } \\
\text { amount of lattice } \\
\text { water remains } \\
\text { unchanged during } \\
\text { cycling (TGA) }\end{array}$ & S142 \\
\hline $\begin{array}{l}\text { a) } \alpha-V_{2} O_{5} \text { b) amorphous } \\
\mathrm{V}_{2} \mathrm{O}_{5}\end{array}$ & $\begin{array}{l}\text { Room } \\
\text { temperature, } \\
\text { capacitive carbon, } \\
0.3 \mathrm{M} \mathrm{Mg}(\mathrm{TFSI})_{2} \text { in } \\
\text { glyme:diglyme } \\
\text { (1:1 vol. ratio) }\end{array}$ & Not provided & Not provided & 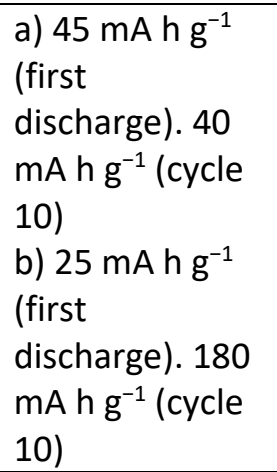 & Not provided & S143 \\
\hline
\end{tabular}




\begin{tabular}{|c|c|c|c|c|c|c|}
\hline Material & $\begin{array}{c}\text { Cycling } \\
\text { conditions: } \\
\text { Temperature, } \\
\text { anode, and } \\
\text { electrolyte } \\
\end{array}$ & $\begin{array}{l}\text { Magnesiated Product } \\
\text { phases }\end{array}$ & Redox changes & $\begin{array}{c}\text { Reported } \\
\text { Electrochemical } \\
\text { Capacity }\end{array}$ & $\begin{array}{c}\text { Experimentally } \\
\text { Verified Magnesiated } \\
\text { Composition }\end{array}$ & Reference \\
\hline $\mathrm{Mn}_{0.04} \mathrm{~V}_{2} \mathrm{O}_{5} \cdot 1.17 \mathrm{H}_{2} \mathrm{O}$ & $\begin{array}{l}\text { Room } \\
\text { temperature, } \\
\text { capacitive carbon, } \\
0.3 \mathrm{M} \mathrm{Mg}(\mathrm{TFSI})_{2} \text { in } \\
\text { acetonitrile }\end{array}$ & $\begin{array}{l}\text { Contraction of layer } \\
\text { axis (c-lattice } \\
\text { parameter) with } \\
\text { discharge. Changes } \\
\text { are reversed during } \\
\text { charge. (in-situ XRD } \\
\text { and HRTEM) }\end{array}$ & $\begin{array}{l}\text { Reversible } \mathrm{V}^{5+} / \mathrm{V}^{4+} \\
\text { surface redox } \\
\text { activity with } \\
\text { discharge and } \\
\text { charge (XPS) }\end{array}$ & $\begin{array}{l}142 \mathrm{~mA} \mathrm{~h} \mathrm{~g}^{-1} \\
\text { (first } \\
\text { discharge), } 130 \\
\mathrm{~mA} \mathrm{~h} \mathrm{~g}^{-1} \text { (cycle } \\
100 \text { ) }\end{array}$ & $\begin{array}{l}\text { Mg present in } \\
\text { discharged electrode, } \\
\text { and is absent in } \\
\text { charged electrode } \\
\text { (XPS) Quantification } \\
\text { not given. }\end{array}$ & S144 \\
\hline$\beta-\mathrm{NaV}_{6} \mathrm{O}_{15}$ & $\begin{array}{l}\text { Room } \\
\text { temperature, } \mathrm{Mg} \\
\text { foil, } 0.5 \mathrm{M} \\
\mathrm{Mg}(\mathrm{TFSI})_{2} \text { in } \mathrm{DME} \\
\text { with a) through } \\
\text { electrolyte drying } \\
\text { steps b) } \\
\text { electrolyte used } \\
\text { as-received } \\
\text { (water content } \\
\text { not specified) }\end{array}$ & $\begin{array}{l}\text { b) Initial expansion in } \\
\text { the } b c \text { plane and } \\
\text { contraction in the } a \\
\text { lattice parameter with } \\
\text { discharge to } 100 \mathrm{~mA} \\
\mathrm{~h} \mathrm{~g}^{-1} \text {, further } \\
\text { discharge to } ~ 215 \mathrm{~mA} \\
\mathrm{~h} \mathrm{~g}^{-1} \text { gave expansion } \\
\text { in the ac plane and } \\
\text { contraction in the } b \\
\text { lattice parameter. } \\
\text { (the unit cell volume } \\
\text { always increased } \\
\text { during discharge). } \\
\text { Changes were largely } \\
\text { reversed upon charge. } \\
\text { (XRD) }\end{array}$ & Not provided & $\begin{array}{l}\text { a) } 155 \mathrm{~mA} \mathrm{~h} \mathrm{~g}{ }^{-1} \\
\text { (first } \\
\text { discharge), } 60 \\
\mathrm{~mA} \mathrm{~h} \mathrm{~g}^{-1} \\
\text { (second cycle). } \\
\text { b) } 270 \mathrm{~mA} \mathrm{~h} \mathrm{~g} \\
\text { (first } \\
\text { discharge), } 125 \\
\mathrm{~mA} \mathrm{~h} \mathrm{~g}{ }^{-1} \text { (cycle } \\
\text { 8) }\end{array}$ & $\begin{array}{l}\text { b) } \mathrm{Mg}_{1.1} \mathrm{NaV}_{6} \mathrm{O}_{15} \\
\text { (discharged), } \mathrm{NaV}_{6} \mathrm{O}_{15} \\
\text { (charged) (EDX) }\end{array}$ & S145 \\
\hline
\end{tabular}




\begin{tabular}{|c|c|c|c|c|c|c|}
\hline Material & $\begin{array}{c}\text { Cycling } \\
\text { conditions: } \\
\text { Temperature, } \\
\text { anode, and } \\
\text { electrolyte } \\
\end{array}$ & $\begin{array}{l}\text { Magnesiated Product } \\
\text { phases }\end{array}$ & Redox changes & $\begin{array}{l}\text { Reported } \\
\text { Electrochemical } \\
\text { Capacity }\end{array}$ & $\begin{array}{c}\text { Experimentally } \\
\text { Verified Magnesiated } \\
\text { Composition }\end{array}$ & Reference \\
\hline $\mathrm{V}_{4} \mathrm{Nb}_{18} \mathrm{O}_{55}$ & $\begin{array}{l}110^{\circ} \mathrm{C} \text {, capacitive } \\
\text { carbon, } 0.5 \mathrm{M} \\
\mathrm{Mg}(\mathrm{TFSI})_{2} \text { in } \\
\mathrm{PY}_{14} \mathrm{TFSI}(43.7 \\
\text { ppm water) }\end{array}$ & $\begin{array}{l}\text { Expansion of the } a, b \\
\text { and } c \text { lattice } \\
\text { parameters on } \\
\text { discharge. Contraction } \\
\text { of the } b \text { and } c \\
\text { parameters on } \\
\text { charge. (XRD) }\end{array}$ & $\begin{array}{l}\text { Reduction of } \mathrm{V}^{5+} \text { to } \\
\mathrm{V}^{3+} \text {, and partial } \\
\text { reduction of } \mathrm{Nb}^{5+} \text { to } \\
\mathrm{Nb}^{4+} \text { on discharge. } \\
\mathrm{Oxidation} \text { of } \mathrm{V}^{3+} \text { to } \\
\mathrm{V}^{4+} \text { and } \mathrm{Nb}^{4+} \text { to } \mathrm{Nb}^{5+} \\
\text { on charge. (XAS) }\end{array}$ & $\begin{array}{l}70 \mathrm{~mA} \mathrm{~h} \mathrm{~g}^{-1} \\
\text { (first } \\
\text { discharge), } 50 \\
\mathrm{~mA} \mathrm{~h} \mathrm{~g}^{-1} \text { (cycle } \\
\text { 50) }\end{array}$ & $\begin{array}{l}\mathrm{Mg}_{4} \mathrm{~V}_{4} \mathrm{Nb}_{18} \mathrm{O}_{55} \\
\text { (discharged), } \\
\mathrm{Mg}_{0.13} \mathrm{~V}_{4} \mathrm{Nb}_{18} \mathrm{O}_{55} \\
\text { (charged) }\end{array}$ & S146 \\
\hline $\mathrm{Mg}\left(\mathrm{Mg}_{0.5} \mathrm{~V}_{1.4} \mathrm{Ni}_{0.1}\right) \mathrm{O}_{4}$ & $\begin{array}{l}90{ }^{\circ} \mathrm{C}, \mathrm{AZ31} \text { alloy, } \\
1 \mathrm{M} \\
\mathrm{Mg}\left[\mathrm{N}\left(\mathrm{SO}_{2} \mathrm{CF}_{3}\right)_{2}\right]_{2} \text { in } \\
\text { triglyme }\end{array}$ & Not provided & $\begin{array}{l}\text { Oxidation of } \mathrm{V}^{3.3+} \text { on } \\
\text { charge (not } \\
\text { quantified). } \\
\text { Oxidation state of } \\
\mathrm{Ni}^{2+} \text { did not change. } \\
\text { (XAS) }\end{array}$ & 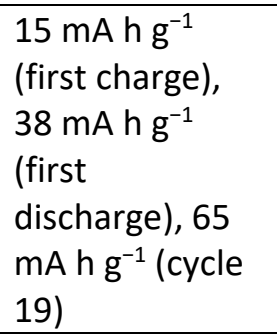 & Not provided & S147 \\
\hline
\end{tabular}




\begin{tabular}{|c|c|c|c|c|c|c|}
\hline Material & $\begin{array}{c}\text { Cycling } \\
\text { conditions: } \\
\text { Temperature, } \\
\text { anode, and } \\
\text { electrolyte }\end{array}$ & $\begin{array}{l}\text { Magnesiated Product } \\
\text { phases }\end{array}$ & Redox changes & $\begin{array}{c}\text { Reported } \\
\text { Electrochemical } \\
\text { Capacity }\end{array}$ & $\begin{array}{c}\text { Experimentally } \\
\text { Verified Magnesiated } \\
\text { Composition }\end{array}$ & Reference \\
\hline $\mathrm{Mg}_{4} \mathrm{~V}_{4.7} \mathrm{Ni}_{0.3} \mathrm{O}_{12}$ & $\begin{array}{l}90^{\circ} \mathrm{C}, \mathrm{AZ31} \text { alloy, } \\
1 \mathrm{M} \\
\mathrm{Mg}\left[\mathrm{N}\left(\mathrm{SO}_{2} \mathrm{CF}_{3}\right)_{2}\right]_{2} \text { in } \\
\text { triglyme }\end{array}$ & $\begin{array}{l}\text { Mg removed from 8a } \\
\text { sites and increased } \\
\text { occupation on } 16 \mathrm{c} \\
\text { sites on charge. } \\
\text { Subsequent discharge } \\
\text { increases occupation } \\
\text { of } \mathrm{Mg} \text { on both } 8 \mathrm{a} \text { and } \\
\text { 16c sites. This is } \\
\text { observed again during } \\
\text { a repeat cycle, with a } \\
\text { greater proportion of } \\
\text { Mg occupying } 16 \mathrm{c} \\
\text { sites after the } 2^{\text {nd }} \\
\text { discharge compared } \\
\text { to the first discharge } \\
\text { (XRD). }\end{array}$ & $\begin{array}{l}\text { Oxidation of } \mathrm{V}^{3.3+} \text { on } \\
\text { charge, reduction } \\
\text { on discharge (not } \\
\text { quantified). } \\
\text { Oxidation state of } \\
\mathrm{Ni}^{2+} \text { did not change } \\
\text { during the first } \\
\text { cycle, but was } \\
\text { oxidized/reduced } \\
\text { with } \\
\text { charge/discharge } \\
\text { respectively on the } \\
\text { second cycle (XAS) }\end{array}$ & $\begin{array}{l}130 \mathrm{~mA} \mathrm{~h} \mathrm{~g}^{-1} \\
\text { (first charge), } \\
195 \mathrm{~mA} \mathrm{~h} \mathrm{~g}{ }^{-1} \\
\text { (first } \\
\text { discharge). }\end{array}$ & $\begin{array}{l}\mathrm{Mg}_{3} \mathrm{~V}_{4.7} \mathrm{Ni}_{0.3} \mathrm{O}_{12} \\
\text { (charged), } \\
\mathrm{Mg}_{4} \mathrm{~V}_{4.7} \mathrm{Ni}_{0.3} \mathrm{O}_{12} \\
\text { (discharged) (XRD } \\
\text { Rietveld refinement) }\end{array}$ & S148 \\
\hline$\alpha-V_{2} O_{5}$ & $\begin{array}{l}\text { Room } \\
\text { temperature, } \\
\mathrm{Mg}_{x} \mathrm{Mo}_{6} \mathrm{~S}_{8}, 1 \mathrm{M} \\
\mathrm{Mg}\left(\mathrm{ClO}_{4}\right)_{2} \text { in } \\
\text { acetonitrile }\end{array}$ & $\begin{array}{l}\text { Smooth, reversible } \\
\text { changes in crystal } \\
\text { structure with } \\
\text { discharge and charge. } \\
\text { Discharged phase has } \\
\text { great similarity to } \beta \text { - } \\
\mathrm{H}_{\mathrm{x}} \mathrm{V}_{2} \mathrm{O}_{5} \text { (XRD) }\end{array}$ & $\begin{array}{l}\text { Reversible } \\
\text { reduction/oxidation } \\
\text { of the } \mathrm{V}^{5+} / \mathrm{V}^{4+} \text { redox } \\
\text { couple with } \\
\text { discharge and } \\
\text { charge (XAS) }\end{array}$ & $\begin{array}{l}102 \mathrm{~mA} \mathrm{~h} \mathrm{~g}^{-1} \\
\text { (first } \\
\text { discharge), } 38 \\
\mathrm{~mA} \mathrm{~h} \mathrm{~g}^{-1} \text { (cycle } \\
100 \text { ) }\end{array}$ & Not provided & S149 \\
\hline
\end{tabular}




\begin{tabular}{|c|c|c|c|c|c|c|}
\hline Material & $\begin{array}{c}\text { Cycling } \\
\text { conditions: } \\
\text { Temperature, } \\
\text { anode, and } \\
\text { electrolyte }\end{array}$ & $\begin{array}{l}\text { Magnesiated Product } \\
\text { phases }\end{array}$ & Redox changes & $\begin{array}{c}\text { Reported } \\
\text { Electrochemical } \\
\text { Capacity }\end{array}$ & $\begin{array}{c}\text { Experimentally } \\
\text { Verified Magnesiated } \\
\text { Composition }\end{array}$ & Reference \\
\hline $\begin{array}{l}\text { a) Amorphous hydrated } \\
\mathrm{V}_{2} \mathrm{O}_{5} \text { (thin film), b) } \alpha- \\
\mathrm{V}_{2} \mathrm{O}_{5} \text { (thin film) }\end{array}$ & $\begin{array}{l}\text { Room } \\
\text { temperature, } \\
\text { capacitive carbon, } \\
0.1 \mathrm{M} \mathrm{Mg}\left(\mathrm{ClO}_{4}\right)_{2} \\
\text { in } \mathrm{PC} \text { with } 0.6 \mathrm{M} \\
\text { water }\end{array}$ & Not provided & Not provided & $\begin{array}{l}\text { a) } 220 \mathrm{~mA} \mathrm{~h} \mathrm{~g}^{-1} \\
\text { (first } \\
\text { discharge), } 105 \\
\mathrm{~mA} \mathrm{~h} \mathrm{~g}{ }^{-1} \text { (cycle } \\
\text { 4). b) } 30 \mathrm{~mA} \mathrm{~h} \\
\mathrm{~g}^{-1} \text { (first } \\
\text { discharge), } 25 \\
\mathrm{~mA} \mathrm{~h} \mathrm{~g}{ }^{-1} \text { (cycle } \\
\text { 4) }\end{array}$ & Not provided & S150 \\
\hline$\alpha-V_{2} O_{5}$ & $\begin{array}{l}\text { Room } \\
\text { temperature, } \\
\text { capacitive carbon, } \\
0.2 \mathrm{M} \mathrm{Mg}\left(\mathrm{ClO}_{4}\right)_{2} \\
\text { solution in } \\
\text { acetonitrile }\end{array}$ & $\begin{array}{l}\text { New } \mathrm{XRD} \text { peaks } \\
\text { appear on discharge, } \\
\text { referenced to } \\
\mathrm{Mg}_{0.45} \mathrm{~V}_{2} \mathrm{O}_{5} \text { (reference } \\
\text { pattern not } \\
\text { definitively } \\
\text { magnesiated } \mathrm{V}_{2} \mathrm{O}_{5}, \mathrm{Mg} \\
\text { intercalation was not } \\
\text { verified in that } \\
\text { report). Some loss of } \\
\text { crystallinity on } \\
\text { discharge (Raman } \\
\text { spectroscopy) }\end{array}$ & Not provided & $\begin{array}{l}225 \mathrm{~mA} \mathrm{~h} \mathrm{~g}^{-1} \\
\text { (first } \\
\text { discharge). }\end{array}$ & $\begin{array}{l}\mathrm{Mg}_{0.35} \mathrm{~V}_{2} \mathrm{O}_{5} \\
\text { (discharged) (ICP-AES) }\end{array}$ & S151 \\
\hline
\end{tabular}




\begin{tabular}{|c|c|c|c|c|c|c|}
\hline Material & $\begin{array}{c}\text { Cycling } \\
\text { conditions: } \\
\text { Temperature, } \\
\text { anode, and } \\
\text { electrolyte } \\
\end{array}$ & $\begin{array}{l}\text { Magnesiated Product } \\
\text { phases }\end{array}$ & Redox changes & $\begin{array}{c}\text { Reported } \\
\text { Electrochemical } \\
\text { Capacity }\end{array}$ & $\begin{array}{c}\text { Experimentally } \\
\text { Verified Magnesiated } \\
\text { Composition }\end{array}$ & Reference \\
\hline $\mathrm{MgV}_{2} \mathrm{O}_{4}$ & $\begin{array}{l}\text { a) } 110{ }^{\circ} \mathrm{C} \text {, } \\
\text { capacitive carbon, } \\
0.5 \mathrm{M} \mathrm{Mg}(\mathrm{TFSI})_{2} \text { in } \\
\mathrm{PY}_{14} \mathrm{TFSI}(43 \mathrm{ppm} \\
\text { water }) . \mathrm{b}) 85^{\circ} \mathrm{C} \text {, } \\
\mathrm{Mg} \text { foil, } 0.1 \mathrm{M} \\
\mathrm{Mg}(\mathrm{TPFA})_{2} \text { in } \\
\text { triglyme }\end{array}$ & $\begin{array}{l}\text { a) Contraction of unit } \\
\text { cell on charge, partial } \\
\text { re-expansion on } \\
\text { discharge. Partial loss } \\
\text { of crystallinity after } \\
\text { cycling. (XRD). } \\
\text { Disappearance and re- } \\
\text { emergence of Mg in } \\
\text { tetrahedral sites with } \\
\text { discharge and charge, } \\
\text { respectively ( }{ }^{25} \mathrm{Mg}- \\
\text { NMR). Spinel } \\
\text { structure preserved } \\
\text { with cycling (STEM). }\end{array}$ & $\begin{array}{l}\text { a) Partial oxidation } \\
\text { of } \mathrm{V}^{3+} \text { to } \mathrm{V}^{4+} \text { on } \\
\text { charging, reduction } \\
\text { to } \mathrm{V}^{3+} \text { on discharge. } \\
\text { (XAS) }\end{array}$ & $\begin{array}{l}\text { a) } 285 \mathrm{~mA} \mathrm{~h} \mathrm{~g}^{-1} \\
\text { (first charge), } \\
235 \mathrm{~mA} \mathrm{~h} \mathrm{~g}^{-1} \\
\text { (first } \\
\text { discharge). } 150 \\
\mathrm{~mA} \mathrm{~h} \mathrm{~g}^{-1} \text { (cycle } \\
\text { 15). } \\
\text { b) } 120 \mathrm{~mA} \mathrm{~h} \mathrm{~g}^{-1} \\
\text { (first charge), } \\
170 \mathrm{~mA} \mathrm{~h} \mathrm{~g}^{-1} \\
\text { (first } \\
\text { discharge), } 215 \\
\mathrm{~mA} \mathrm{~h} \mathrm{~g}{ }^{-1} \\
\text { (second } \\
\text { charge), } 150 \\
\mathrm{~mA} \mathrm{~h} \mathrm{~g}{ }^{-1} \\
\text { (second } \\
\text { discharge) }\end{array}$ & $\begin{array}{l}\text { a) } \mathrm{Mg}_{0.3} \mathrm{~V}_{2} \mathrm{O}_{4} \\
\text { (charged), } \mathrm{Mg}_{1.11} \mathrm{~V}_{2} \mathrm{O}_{4} \\
\text { (discharged) (EDX) }\end{array}$ & S152 \\
\hline
\end{tabular}




\begin{tabular}{|c|c|c|c|c|c|c|}
\hline Material & $\begin{array}{c}\text { Cycling } \\
\text { conditions: } \\
\text { Temperature, } \\
\text { anode, and } \\
\text { electrolyte } \\
\end{array}$ & $\begin{array}{c}\text { Magnesiated Product } \\
\text { phases }\end{array}$ & Redox changes & $\begin{array}{c}\text { Reported } \\
\text { Electrochemical } \\
\text { Capacity }\end{array}$ & $\begin{array}{c}\text { Experimentally } \\
\text { Verified Magnesiated } \\
\text { Composition }\end{array}$ & Reference \\
\hline $\mathrm{Mg}\left(\mathrm{Mg}_{0.5} \mathrm{~V}_{1.5}\right) \mathrm{O}_{4}$ & $\begin{array}{l}\text { Room } \\
\text { temperature, } \\
\text { capacitive carbon, } \\
0.3 \mathrm{M} \mathrm{Mg}(\mathrm{TFSI})_{2} \text { in } \\
\text { acetonitrile }(55.9 \\
\text { ppm water) }\end{array}$ & $\begin{array}{l}\text { Negligible shifts in } \\
\text { diffraction peaks and } \\
\text { lattice spacing with } \\
\text { discharge and charge. } \\
\text { Possible emergence of } \\
\mathrm{Mg}_{0.5} \mathrm{~V}_{1.5} \mathrm{O}_{4} \text { phase } \\
\text { (two phase reaction) } \\
\text { on discharge } \\
\text { (XRD/TEM) }\end{array}$ & $\begin{array}{l}\text { Oxidation of mixed } \\
\mathrm{V}^{3+} / \mathrm{V}^{4+} \text { to majority } \\
\mathrm{V}^{4+} \text { at the surface } \\
\text { on charge, } \\
\text { reduction to } \mathrm{V}^{3+} \text { on } \\
\text { discharge (XPS). } \\
\text { Some oxidation was } \\
\text { evident in the bulk } \\
\text { on charge, but only } \\
\text { partial reduction } \\
\text { observed on } \\
\text { discharge (XAS) }\end{array}$ & $\begin{array}{l}250 \mathrm{~mA} \mathrm{~h} \mathrm{~g}^{-1} \\
\text { (first charge), } \\
225 \mathrm{~mA} \mathrm{~h} \mathrm{~g}^{-1} \\
\text { (cycle 100) }\end{array}$ & $\begin{array}{l}\mathrm{Mg}_{0.5} \mathrm{~V}_{1.5} \mathrm{O}_{4} \text { (charged), } \\
\mathrm{Mg}\left(\mathrm{Mg}_{0.44}\right) \mathrm{V}_{1.5} \mathrm{O}_{4} \\
\text { (discharged) (ICP-OES) }\end{array}$ & S153 \\
\hline $\mathrm{V}_{2} \mathrm{O}_{3} /$ Graphene Oxide & $\begin{array}{l}\text { Room } \\
\text { temperature, } \\
\text { capacitive carbon, } \\
0.3 \mathrm{M} \mathrm{Mg}(\mathrm{TFSI})_{2} \text { in } \\
\text { acetonitrile }\end{array}$ & $\begin{array}{l}\text { Unit cell expansion on } \\
\text { discharge, contraction } \\
\text { on recharge (XRD) }\end{array}$ & $\begin{array}{l}\text { Reduction of } \\
\text { surface } \mathrm{V}^{3+} \text { to } \mathrm{V}^{2+} \text { on } \\
\text { discharge, which } \\
\text { re-oxidized to } \mathrm{V}^{3+} \\
\text { on charge (XPS) }\end{array}$ & $\begin{array}{l}285 \mathrm{~mA} \mathrm{~h} \mathrm{~g}^{-1} \\
\text { (first } \\
\text { discharge), } 220 \\
\mathrm{~mA} \mathrm{~h} \mathrm{~g}^{-1} \text { (cycle } \\
100 \text { ) }\end{array}$ & $\begin{array}{l}\text { Mg well dispersed in } \\
\text { discharged particles, } \\
\text { but quantification not } \\
\text { provided (EDS) }\end{array}$ & S155 \\
\hline
\end{tabular}




\begin{tabular}{|c|c|c|c|c|c|c|}
\hline Material & $\begin{array}{c}\text { Cycling } \\
\text { conditions: } \\
\text { Temperature, } \\
\text { anode, and } \\
\text { electrolyte }\end{array}$ & $\begin{array}{l}\text { Magnesiated Product } \\
\text { phases }\end{array}$ & Redox changes & $\begin{array}{c}\text { Reported } \\
\text { Electrochemical } \\
\text { Capacity }\end{array}$ & $\begin{array}{c}\text { Experimentally } \\
\text { Verified Magnesiated } \\
\text { Composition }\end{array}$ & Reference \\
\hline$\zeta-\mathrm{V}_{2} \mathrm{O}_{5}$ & $\begin{array}{l}110^{\circ} \mathrm{C} \text {, capacitive } \\
\text { carbon, } 0.5 \mathrm{M} \\
\mathrm{Mg}(\mathrm{TFSI})_{2} \text { in } \\
\mathrm{PY}_{14} \mathrm{TFSI} \text { with a) } \\
43.7 \mathrm{ppm} \text { water } \\
\text { b) } 2823 \mathrm{ppm} \\
\text { water }\end{array}$ & $\begin{array}{l}\text { a,b) Anisotropic } \\
\text { expansion of unit cell, } \\
\text { where lattice } \\
\text { parameter } a \text { showed } \\
\text { the largest expansion } \\
\text { increase compared to } \\
b \text { and } c \text {, on discharge. } \\
\text { Changes were largely } \\
\text { reversed on charge. } \\
(\mathrm{XRD})\end{array}$ & $\begin{array}{l}\text { Greater reduction } \\
\text { of } V \text { in wet } \\
\text { electrolyte (b) than } \\
\text { dry electrolyte (a). } \\
\text { Original oxidation } \\
\text { state recovered on } \\
\text { charge. (XAS). }\end{array}$ & $\begin{array}{l}\mathrm{a}, \mathrm{b}) 300 \mathrm{~mA} \mathrm{~h} \\
\mathrm{~g}^{-1} \text { (first and } \\
24^{\text {th }} \text { discharge) }\end{array}$ & $\begin{array}{l}\text { a) } \mathrm{Mg}_{0.36} \mathrm{~V}_{2} \mathrm{O}_{5} \\
\text { (discharged), } \\
\mathrm{Mg}_{0.09} \mathrm{~V}_{2} \mathrm{O}_{5} \text { (charged) } \\
\text { b) } \mathrm{Mg}_{0.29} \mathrm{~V}_{2} \mathrm{O}_{5} \\
\text { (discharged), } \\
\mathrm{Mg}_{0.11} \mathrm{~V}_{2} \mathrm{O}_{5} \text { (charged) } \\
\text { (EDS) }\end{array}$ & S156 \\
\hline$\zeta-\mathrm{V}_{2} \mathrm{O}_{5}$ & $\begin{array}{l}110^{\circ} \mathrm{C} \text {, capacitive } \\
\text { carbon, } 0.5 \mathrm{M} \\
\mathrm{Mg}(\mathrm{TFSI})_{2} \text { in } \\
\mathrm{PY}_{14} \mathrm{TFSI} \text { with } 43 \\
\text { ppm water }\end{array}$ & $\begin{array}{l}\text { Discharge gave two } \\
\mathrm{C} 2 / \mathrm{m} \text { structures } \\
\text { assigned to } \mathrm{Mg} \text {-rich } \\
\text { and } \mathrm{Mg} \text {-poor phases } \\
\text { (both structures had } \\
\text { increased unit cell } \\
\text { volume compared to } \\
\text { the pristine } \zeta-\mathrm{V}_{2} \mathrm{O}_{5} \text { ). } \\
\text { The original structure } \\
\text { and lattice } \\
\text { parameters were } \\
\text { almost entirely } \\
\text { regained on charge } \\
\text { (XRD) }\end{array}$ & $\begin{array}{l}\text { Partial reduction of } \\
\mathrm{V}^{5+} \text { to } \mathrm{V}^{4+} \text { on } \\
\text { discharge, and this } \\
\text { was partially } \\
\text { reversed upon } \\
\text { charge (XAS/EELS) }\end{array}$ & $\begin{array}{l}103 \mathrm{~mA} \mathrm{~h} \mathrm{~g}^{-1} \\
\text { (first } \\
\text { discharge), } 122 \\
\mathrm{~mA} \mathrm{~h} \mathrm{~g}{ }^{-1} \text { (cycle } \\
15 \text { ) }\end{array}$ & $\begin{array}{l}\mathrm{Mg}_{0.18(11)} \mathrm{V}_{2} \mathrm{O}_{5} \\
\text { (discharged), } \\
\mathrm{Mg}_{0.09(5)} \mathrm{V}_{2} \mathrm{O}_{5} \text { (charged) }\end{array}$ & S157 \\
\hline
\end{tabular}




\section{References}

(1) Bayliss, R. D.; Key, B.; Sai Gautam, G.; Canepa, P.; Kwon, B. J.; Lapidus, S. H.; Dogan, F.; Adil, A. A.; Lipton, A. S.; Baker, P. J.; Ceder, G.; Vaughey, J. T.; Cabana, J. Probing Mg Migration in Spinel Oxides. Chem. Mater. 2020, 32 (15), 663-670.

(2) Hu, L.; Johnson, I. D.; Kim, S.; Nolis, G. M.; Freeland, J.; Yoo, H. D.; Fister, T. T.; McCafferty, L.; Ashton, T. E.; Darr, J. A.; Cabana, J. Tailoring the Electrochemical Activity of Magnesium Chromium Oxide Towards Mg Batteries Through Control of Size and Crystal Structure. Nanoscale 2019, 11, 639-646.

(3) Kwon, B. J.; Lau, K. C.; Park, H.; Wu, Y. A.; Hawthorne, K. L.; Li, H.; Kim, S.; Bolotin, I. L.; Fister, T. T.; Zapol, P.; Klie, R. F.; Cabana, J.; Liao, C.; Lapidus, S. H.; Key, B.; Vaughey, J. T. Probing Electrochemical Mg-lon Activity in $\mathrm{MgCr}_{2-\mathrm{x}} \mathrm{V}_{\mathrm{x}} \mathrm{O}_{4}$ Spinel Oxides. Chem. Mater. 2020, 32 (3), $1162-1171$.

(4) Kwon, B. J.; Yin, L.; Park, H.; Parajuli, P.; Kumar, K.; Kim, S.; Yang, M.; Murphy, M.; Zapol, P.; Liao, C.; Fister, T. T.; Klie, R. F.; Cabana, J.; Vaughey, J. T.; Lapidus, S. H.; Key, B. High Voltage Mg-Ion Battery Cathode via a Solid Solution Cr-Mn Spinel Oxide. Chem. Mater. 2020, 32 (15), 6577-6587.

(5) Ichitsubo, T.; Adachi, T.; Yagi, S.; Doi, T. Potential Positive Electrodes for High-Voltage Magnesium-lon Batteries. J. Mater. Chem. 2011, 21 (32), 11764-11772.

(6) Okamoto, S.; Ichitsubo, T.; Kawaguchi, T.; Kumagai, Y.; Oba, F.; Yagi, S.; Shimokawa, K.; Goto, N.; Doi, T.; Matsubara, E. Intercalation and Push-Out Process with Spinel-to-Rocksalt Transition on Mg Insertion into Spinel Oxides in Magnesium Batteries. Adv. Sci. 2015, 2 (8), 1500072.

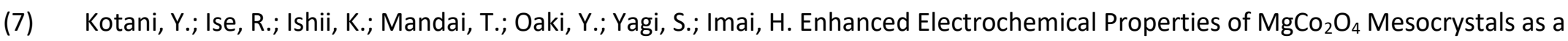
Positive Electrode Active Material for Mg Batteries. J. Alloys Compd. 2018, 739, 793-798.

(8) Sa, N.; Mukherjee, A.; Han, B.; Ren, Y.; Klie, R. F.; Key, B.; Vaughey, J. T. Direct Observation of MgO Formation at Cathode Electrolyte Interface of a Spinel $\mathrm{MgCO}_{2} \mathrm{O}_{4}$ Cathode upon Electrochemical Mg Removal and Insertion. J. Power Sources 2019, 424, 68-75.

(9) Shimokawa, K.; Atsumi, T.; Harada, M.; Ward, R. E.; Nakayama, M.; Kumagai, Y.; Oba, F.; Okamoto, N. L.; Kanamura, K.; Ichitsubo, T. ZincBased Spinel Cathode Materials for Magnesium Rechargeable Batteries: Toward the Reversible Spinel-Rocksalt Transition. J. Mater. Chem. A 2019, 7 (19), 12225-12235.

(10) Truong, Q. D.; Kobayashi, H.; Honma, I. Rapid Synthesis of $\mathrm{MgCo}_{2} \mathrm{O}_{4}$ and $\mathrm{Mg}_{2 / 3} \mathrm{Ni}_{4 / 3} \mathrm{O}_{2}$ Nanocrystals in Supercritical Fluid for $\mathrm{Mg}$-lon Batteries. RSC Adv. 2019, 9 (63), 36717-36725.

(11) Idemoto, Y.; Mizutani, Y.; Ishibashi, C.; Ishida, N.; Kitamura, N. Synthesis, Crystal Structure and Electrode Properties of Spinel-Type $\mathrm{MgCO}_{2-\mathrm{x}} \mathrm{Mn}_{\times} \mathrm{O}_{4}$. Electrochemistry 2019, 87 (4), 220-228. 


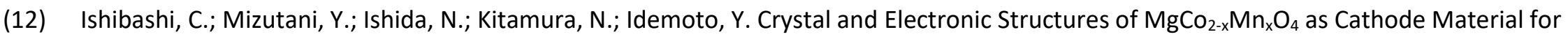
Magnesium Secondary Batteries Using First-Principles Calculations and Quantum Beam Measurements. Bull. Chem. Soc. Jpn. 2019, 92 (12), 1950-1959.

(13) Idemoto, Y.; Ichiyama, M.; Ishida, N.; Kitamura, N. Structural and Electronic Properties of Spinel Type $\mathrm{Mg}_{1+y} \mathrm{Co}_{2-x-y} \mathrm{Mn}_{x} \mathrm{O}_{4}$ for Cathode Applications in Magnesium Rechargeable Batteries. J. Power Sources 2021, 482, 228920.

(14) Novák, P. Electrochemical Insertion of Magnesium in Metal Oxides and Sulfides from Aprotic Electrolytes. J. Electrochem. Soc. 1993, 140 (1), 140.

(15) Nuli, Y.; Zheng, Y.; Wang, Y.; Yang, J.; Wang, J. Electrochemical Intercalation of Mg2+ in 3D Hierarchically Porous Magnesium Cobalt Silicate and Its Application as an Advanced Cathode Material in Rechargeable Magnesium Batteries. J. Mater. Chem. 2011, 21 (33), 12437-12443.

(16) Zheng, Y.; Nuli, Y.; Chen, Q.; Wang, Y.; Yang, J.; Wang, J. Magnesium Cobalt Silicate Materials for Reversible Magnesium lon Storage. Electrochim. Acta 2012, 66, 75-81.

(17) Chen, X.; Bleken, F. L.; Løvvik, O. M.; Vullum-Bruer, F. Comparing Electrochemical Performance of Transition Metal Silicate Cathodes and Chevrel Phase $\mathrm{Mo}_{6} \mathrm{~S}_{8}$ in the Analogous Rechargeable Mg-lon Battery System. J. Power Sources 2016, 321, 76-86.

(18) Li, Y.; Nuli, Y. N.; Yang, J.; Yilinuer, T.; Wang, J. L. MgFeSiO4 Prepared via a Molten Salt Method as a New Cathode Material for Rechargeable Magnesium Batteries. Chinese Sci. Bull. 2011, 56 (4-5), 386-390.

(19) Huang, Z. D.; Masese, T.; Orikasa, Y.; Mori, T.; Minato, T.; Tassel, C.; Kobayashi, Y.; Kageyama, H.; Uchimoto, Y. MgFePO ${ }_{4} F$ as a Feasible Cathode Material for Magnesium Batteries. J. Mater. Chem. A 2014, 2 (30), 11578-11582.

(20) Orikasa, Y.; Kisu, K.; Iwama, E.; Naoi, W.; Yamaguchi, Y.; Yamaguchi, Y.; Okita, N.; Ohara, K.; Munesada, T.; Hattori, M.; Yamamoto, K.; Rozier, P.; Simon, P.; Naoi, K. Noncrystalline Nanocomposites as a Remedy for the Low Diffusivity of Multivalent lons in Battery Cathodes. Chem. Mater. 2020, 32 (3), 1011-1021.

(21) Watanabe, A.; Yamamoto, K.; Orikasa, Y.; Masese, T.; Mori, T.; Uchiyama, T.; Matsunaga, T.; Uchimoto, Y. Reaction Mechanism of Electrochemical Insertion/Extraction of Magnesium lons in Olivine-Type $\mathrm{FePO}_{4}$. Solid State lonics 2020, 349 (December 2019 ), 115311.

(22) Le Poul, N.; Baudrin, E.; Morcrette, M.; Gwizdala, S.; Masquelier, C.; Tarascon, J. M. Development of Potentiometric lon Sensors Based on Insertion Materials as Sensitive Element. Solid State lonics 2003, 159 (1-2), 149-158.

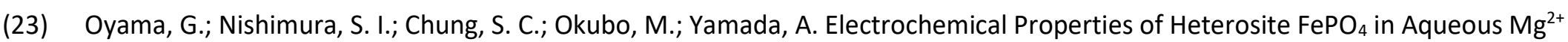
Electrolytes. Electrochemistry 2014, 82 (10), 855-858. 
(24) Zhang, R.; Ling, C. Unveil the Chemistry of Olivine $\mathrm{FePO}_{4}$ as Magnesium Battery Cathode. ACS Appl. Mater. Interfaces 2016, 8 (28), 1801818026.

(25) Song, J.; Noked, M.; Gillette, E.; Duay, J.; Rubloff, G.; Lee, S. B. Activation of a MnO 2 Cathode by Water-Stimulated Mg ${ }^{2+}$ Insertion for a Magnesium lon Battery . Phys. Chem. Chem. Phys. 2015, 17 (7), 5256-5264.

(26) Sahadeo, E.; Song, J.; Gaskell, K.; Kim, N.; Rubloff, G.; Lee, S. B. Investigation of the Water-Stimulated Mg ${ }^{2+}$ Insertion Mechanism in an Electrodeposited $\mathrm{MnO}_{2}$ Cathode Using X-Ray Photoelectron Spectroscopy. Phys. Chem. Chem. Phys. 2018, 20 (4), $2517-2526$.

(27) Rasul, S.; Suzuki, S.; Yamaguchi, S.; Miyayama, M. High Capacity Positive Electrodes for Secondary Mg-lon Batteries. Electrochim. Acta 2012, 82, 243-249.

(28) Sun, X.; Duffort, V.; Mehdi, B. L.; Browning, N. D.; Nazar, L. F. Investigation of the Mechanism of Mg Insertion in Birnessite in Nonaqueous and Aqueous Rechargeable Mg-Ion Batteries. Chem. Mater. 2016, 28 (2), 534-542.

(29) Yin, J.; Takeuchi, E. S.; Takeuchi, K. J.; Marschilok, A. C. Synthetic Control of Manganese Birnessite: Impact of Crystallite Size on Li, Na, and Mg Based Electrochemistry. Inorganica Chim. Acta 2016,

(30) Nam, K. W.; Kim, S.; Lee, S.; Salama, M.; Shterenberg, I.; Gofer, Y.; Kim, J. S.; Yang, E.; Park, C. S.; Kim, J. S.; Lee, S. S.; Chang, W. S.; Doo, S. G.; Jo, Y. N.; Jung, Y.; Aurbach, D.; Choi, J. W. The High Performance of Crystal Water Containing Manganese Birnessite Cathodes for Magnesium Batteries. Nano Lett. 2015, 15 (6), 4071-4079.

(31) Wu, C.; Zhao, G.; Bao, X.; Chen, X.; Sun, K. Hierarchically Porous Delta-Manganese Dioxide Films Prepared by an Electrochemically Assistant Method for Mg Ion Battery Cathodes with High Rate Performance. Journal of Alloys and Compounds. 2019, pp 914-919.

(32) Wang, M.; Yagi, S. Layered Birnessite $\mathrm{MnO}_{2}$ with Enlarged Interlayer Spacing for Fast Mg-lon Storage. J. Alloys Compd. $2020,820,153135$.

(33) Li, Y.; Xu, D.; Zhang, D.; Wei, Y.; Zhang, R.; Guo, Y. Study on $\mathrm{MnO}_{2} / \mathrm{MXene-} \mathrm{Ti}_{3} \mathrm{C}_{2}$ Composite Materials as Cathode Materials for Magnesium Batteries. RSC Adv. 2019, 9 (58), 33572-33577.

(34) Kwon, B. J.; Kim, C.; Jokisaari, J. R.; Yoo, H. D.; Han, S.-D.; Lau, K.; Liu, Y.; Guo, J.; Key, B.; Klie, R. F.; Cabana, J.; Kim, S.; Lau, K.; Liu, Y.; Guo, J.; Key, B.; Klie, R. F.; Cabana, J. Intercalation of Mg into a Few-Layer Phyllomanganate in Non-Aqueous Electrolytes at Room Temperature. Chem. Mater. 2020, 32 (14), 6014-6025.

(35) Kumagai, N.; Komaba, S.; Sakai, H.; Kumagai, N. Preparation of Todorokite-Type Manganese-Based Oxide and Its Application as Lithium and Magnesium Rechargeable Battery Cathode. J. Power Sources 2001, 97-98, 515-517.

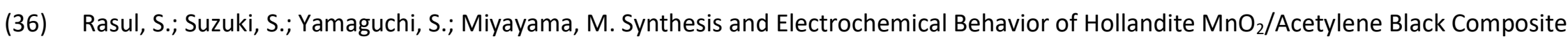
Cathode for Secondary Mg-Ion Batteries. Solid State Ionics 2012, 225, 542-546. 
(37) Zhang, R.; Yu, X.; Nam, K. W.; Ling, C.; Arthur, T. S.; Song, W.; Knapp, A. M.; Ehrlich, S. N.; Yang, X. Q.; Matsui, M. $\alpha-M_{2 n O}$ as a Cathode Material for Rechargeable Mg Batteries. Electrochem. commun. 2012, 23 (1), 110-113.

(38) Zhang, R.; Arthur, T. S.; Ling, C.; Mizuno, F. Manganese Dioxides as Rechargeable Magnesium Battery Cathode; Synthetic Approach to Understand Magnesiation Process. J. Power Sources 2015, 282, 630-638.

(39) Kim, J. S.; Chang, W. S.; Kim, R. H.; Kim, D. Y.; Han, D. W.; Lee, K. H.; Lee, S. S.; Doo, S. G. High-Capacity Nanostructured Manganese Dioxide Cathode for Rechargeable Magnesium Ion Batteries. J. Power Sources 2015, 273, 210-215.

(40) Venkateswarlu, G.; Madhu, D.; Vatsala Rani, J. Graphene/ $\beta-\mathrm{MnO}_{2}$ Composites-Synthesis and Its Electroanalytical Properties Study in the Mg Storage Battery. Int. J. Energy Res. 2020, 44 (13), 10238-10250.

(41) Rasul, S.; Suzuki, S.; Yamaguchi, S.; Miyayama, M. Manganese Oxide Octahedral Molecular Sieves as Insertion Electrodes for Rechargeable Mg Batteries. Electrochim. Acta 2013, 110, 247-252.

(42) NuLi, Y.; Yang, J.; Wang, J.; Li, Y. Electrochemical Intercalation of Mg2+in Magnesium Manganese Silicate and Its Application as HighEnergy Rechargeable Magnesium Battery Cathode. J. Phys. Chem. C 2009, 113 (28), 12594-12597.

(43) Sun, J.; Jiang, Z. Synthesis of Mgmnsio4 and Its Application as Cathode Material for Magnesium Battery. J. New Mater. Electrochem. Syst. 2014, $17(1), 9-11$.

(44) Mori, T.; Masese, T.; Orikasa, Y.; Huang, Z. D.; Okado, T.; Kim, J.; Uchimoto, Y. Anti-Site Mixing Governs the Electrochemical Performances of Olivine-Type $\mathrm{MgMnSiO}_{4}$ Cathodes for Rechargeable Magnesium Batteries. Phys. Chem. Chem. Phys. 2016, 18 (19), $13524-13529$.

(45) Nuli, Y.; Zheng, Y.; Wang, F.; Yang, J.; Minett, A. I.; Wang, J.; Chen, J. MWNT/C/Mg $1.03 \mathrm{Mn}_{0.97} \mathrm{SiO}_{4}$ Hierarchical Nanostructure for Superior Reversible Magnesium Ion Storage. Electrochem. commun. 2011, 13 (10), 1143-1146.

(46) Chen, W.; Zhan, X.; Luo, B.; Ou, Z.; Shih, P. C.; Yao, L.; Pidaparthy, S.; Patra, A.; An, H.; Braun, P. V.; Stephens, R. M.; Yang, H.; Zuo, J. M.; Chen, Q. Effects of Particle Size on $\mathrm{Mg}^{2+}$ Ion Intercalation into $\lambda-\mathrm{MnO}_{2}$ Cathode Materials. Nano Lett. 2019, 19 (7), $4712-4720$.

(47) Kim, C.; Phillips, P. J.; Key, B.; Yi, T.; Nordlund, D.; Yu, Y. S.; Bayliss, R. D.; Han, S. D.; He, M.; Zhang, Z.; Burrell, A. K.; Klie, R. F.; Cabana, J. Direct Observation of Reversible Magnesium Ion Intercalation into a Spinel Oxide Host. Adv. Mater. 2015, 27 (22), $3377-3384$.

(48) Tekin, B.; Demir-Cakan, R. Understanding the Role of Water-Based Electrolytes on Magnesium-Ion Insertion/Extraction into $\lambda$-MnO ${ }_{2}$ Lattice Structure. Solid State Ionics 2019, 335, 67-73.

(49) Truong, Q. D.; Kempaiah Devaraju, M.; Tran, P. D.; Gambe, Y.; Nayuki, K.; Sasaki, Y.; Honma, I. Unravelling the Surface Structure of $\mathrm{MgMn}_{2} \mathrm{O}_{4}$ Cathode Materials for Rechargeable Magnesium-Ion Battery. Chem. Mater. 2017, 29 (15), 6245-6251.

(50) Cabello, M.; Alcántara, R.; Nacimiento, F.; Ortiz, G.; Lavela, P.; Tirado, J. L. Electrochemical and Chemical Insertion/Deinsertion of 
Magnesium in Spinel-Type $\mathrm{MgMn}_{2} \mathrm{O}_{4}$ and Lambda- $\mathrm{MnO}_{2}$ for Both Aqueous and Non-Aqueous Magnesium-lon Batteries. CrystEngComm 2015, $17(45), 8728-8735$.

(51) Truong, Q. D.; Kobayashi, H.; Nayuki, K.; Sasaki, Y.; Honma, I. Atomic-Scale Observation of Phase Transition of $\mathrm{MgMn}_{2} \mathrm{O}_{4} \mathrm{Cubic}_{\mathrm{Spinel}}$ upon the Charging in Mg-lon Battery. Solid State Ionics 2020, 344, 115136.

(52) Ishii, K.; Doi, S.; Ise, R.; Mandai, T.; Oaki, Y.; Yagi, S.; Imai, H. Structured Spinel Oxide Positive Electrodes of Magnesium Rechargeable Batteries: High Rate Performance and High Cyclability by Interconnected Bimodal Pores and Vanadium Oxide Coating. J. Alloys Compd. 2020, 816, 152556.

(53) Feng, Z.; Chen, X.; Qiao, L.; Lipson, A. L.; Fister, T. T.; Zeng, L.; Kim, C.; Yi, T.; Sa, N.; Proffit, D. L.; Burrell, A. K.; Cabana, J.; Ingram, B. J.; Biegalski, M. D.; Bedzyk, M. J.; Fenter, P. Phase-Controlled Electrochemical Activity of Epitaxial Mg-Spinel Thin Films. ACS Appl. Mater. Interfaces 2015, 7 (51), 28438-28443.

(54) Kim, C.; Adil, A. A.; Bayliss, R. D.; Kinnibrugh, T. L.; Lapidus, S. H.; Nolis, G. M.; Freeland, J. W.; Phillips, P. J.; Yi, T.; Yoo, H. D.; Kwon, B. J.; Yu, Y.-S.; Klie, R.; Chupas, P. J.; Chapman, K. W.; Cabana, J. Multivalent Electrochemistry of Spinel $\mathrm{Mg}_{x} \mathrm{Mn}_{3-\mathrm{x}} \mathrm{O}_{4}$ Nanocrystals. Chem. Mater. 2018, 30 (5), 1496-1504.

(55) Zainol, N. H.; Hambali, D.; Osman, Z.; Kamarulzaman, N.; Rusdi, R. Synthesis and Characterization of Ti-Doped MgMn ${ }_{2} \mathrm{O}_{4}$ Cathode Material for Magnesium Ion Batteries. Ionics. 2019, 25 (1), 133-139.

(56) Yokozaki, R.; Kobayashi, H.; Honma, I. Reductive Solvothermal Synthesis of $\mathrm{MgMn}_{2} \mathrm{O}_{4}$ Spinel Nanoparticles for Mg-lon Battery Cathodes. Ceram. Int. 2020, 10236-10241.

(57) Doi, S.; Ise, R.; Mandai, T.; Oaki, Y.; Yagi, S.; Imai, H. Synthesis of Porous Spinel - Type $\mathrm{MgMn}_{2} \mathrm{O}_{4}$ as a Positive Electrode Material for Magnesium Rechargeable Batteries. Langmuir 2020, 36 (29), 8537-8542.

(58) Harudin, N.; Osman, Z.; Majid, S. R.; Othman, L.; Hambali, D.; Silva, M. M. Improved Electrochemical Properties of MgMn2O4 Cathode Materials by Sr Doping for Mg lon Cells. Ionics. 2020. 26, 3947-3958

(59) Hatakeyama, T.; Okamoto, N. L.; Shimokawa, K.; Li, H.; Nakao, A.; Uchimoto, Y.; Tanimura, H.; Kawaguchi, T.; Ichitsubo, T. Electrochemical Phase Transformation Accompanied with Mg Extraction and Insertion in a Spinel $\mathrm{MgMn}_{2} \mathrm{O}_{4}$ Cathode Material. Phys. Chem. Chem. Phys. 2019, 21 (42), 23749-23757.

(60) Yin, J.; Brady, A. B.; Takeuchi, E. S.; Marschilok, A. C.; Takeuchi, K. J. Magnesium-lon Battery-Relevant Electrochemistry of MgMn2O4: Crystallite Size Effects and the Notable Role of Electrolyte Water Content. Chem. Commun. 2017, 53 (26), 3665-3668.

(61) Han, J.; Yagi, S.; Ichitsubo, T. Suppressive Effect of Fe Cations in $\mathrm{Mg}\left(\mathrm{Mn}_{1-\mathrm{x}} \mathrm{Fe}_{\mathrm{x}}\right)_{2} \mathrm{O}_{4}$ Positive Electrodes on Oxidative Electrolyte Decomposition for Mg Rechargeable Batteries. J. Power Sources 2019, 435, 226822. 
(62) Shasha, H.; Yatom, N.; Prill, M.; Zaffran, J.; Biswas, S.; Aurbach, D.; Toroker, M. C.; Ein-Eli, Y. Unveiling lonic Diffusion in $\mathrm{MgNiMnO}_{4}$ Cathode Material for Mg-Ion Batteries via Combined Computational and Experimental Studies. J. Solid State Electrochem. 2019, 23 (11), 3209-3216.

(63) Wang, L.; Asheim, K.; Vullum, P. E.; Svensson, A. M.; Vullum-Bruer, F. Sponge-like Porous Manganese(II,III) Oxide as a Highly Efficient Cathode Material for Rechargeable Magnesium Ion Batteries. Chem. Mater. 2016, 28 (18), 6459-6470.

(64) Wang, L.; Wang, Z.; Vullum, P. E.; Selbach, S. M.; Svensson, A. M.; Vullum-Bruer, F. Solvent-Controlled Charge Storage Mechanisms of Spinel Oxide Electrodes in Mg Organohaloaluminate Electrolytes. Nano Lett. 2018, 18 (2), 763-772.

(65) Sánchez, L.; Pereira-Ramos, J. P. Electrochemical Insertion of Magnesium in a Mixed Manganese-Cobalt Oxide. J. Mater. Chem. 1997, 7 (3), 471-473.

(66) Kim, S.; Nam, K. W.; Lee, S.; Cho, W.; Kim, J. S. J. S.; Kim, B. G.; Oshima, Y.; Kim, J. S. J. S.; Doo, S. G.; Chang, H.; Aurbach, D.; Choi, J. W. Direct Observation of an Anomalous Spinel-to-Layered Phase Transition Mediated by Crystal Water Intercalation. Angew. Chemie - Int. Ed. 2015, 54 (50), 15094-15099.

(67) Kim, S.; Lee, S.; Nam, K. W.; Shin, J.; Lim, S. Y.; Cho, W.; Suzuki, K.; Oshima, Y.; Hirayama, M.; Kanno, R.; Choi, J. W. On the Mechanism of Crystal Water Insertion during Anomalous Spinel-to-Birnessite Phase Transition. Chem. Mater. 2016, 28 (15), 5488-5494.

(68) Miralles, C.; Gómez, R. Proving Insertion of $\mathrm{Mg}$ in $\mathrm{Mn}_{2} \mathrm{O}_{3}$ Electrodes through a Spectroelectrochemical Study. Electrochem. commun. 2019, 106, 106512.

(69) Huang, J.; Poyraz, A. S.; Takeuchi, K. J.; Takeuchi, E. S.; Marschilok, A. C. $\mathrm{M}_{x} \mathrm{Mn}_{8} \mathrm{O}_{16}(\mathrm{M}=\mathrm{Ag}$ or K) as Promising Cathode Materials for Secondary Mg Based Batteries: The Role of the Cation M. Chem. Commun. 2016, 52 (21), 4088-4091.

(70) Poyraz, A. S.; Quilty, C. D.; Housel, L. M.; Hu, X.; Bruck, A. M.; Li, Y. R.; Yin, J.; Zhang, B.; Huang, J.; Wu, L.; Zhu, Y.; Takeuchi, E. S.; Marschilok, A. C.; Takeuchi, K. J. Synthesis and Characterization of $2 \times 4$ Tunnel Structured Manganese Dioxides as Cathodes in Rechargeable Li, Na, and Mg Batteries. J. Electrochem. Soc. 2019, 166 (4), A670-A678.

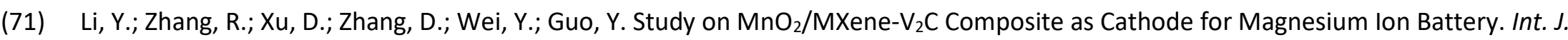
Electrochem. Sci. 2020, 15, 11227-11237.

(72) Spahr, M. E.; Novák, P.; Haas, O.; Nesper, R. Electrochemical Insertion of Lithium, Sodium, and Magnesium in Molybdenum(VI) Oxide. J. Power Sources 1995, 54 (2), 346-351.

(73) Sian, T. S.; Reddy, G. B.; Shivaprasad, S. M. Effect of Microstructure and Stoichiometry on Absorption in Mg Intercalated MoO 3 Thin Films. Electrochem. Solid-State Lett. 2006, 9 (3), 120-123. 
(74) Sian, T. S.; Reddy, G. B.; Shivaprasad, S. M. Study of Mg Ion Intercalation in Polycrystalline $\mathrm{MoO}_{3}$ Thin Films. Japanese J. Appl. Physics, 2004, 43 (9R), 6248-6251.

(75) Gershinsky, G.; Yoo, H. D.; Gofer, Y.; Aurbach, D. Electrochemical and Spectroscopic Analysis of $\mathrm{Mg}^{2+}$ Intercalation into Thin Film Electrodes of Layered Oxides: $\mathrm{V}_{2} \mathrm{O}_{5}$ and $\mathrm{MoO}_{3}$. Langmuir 2013, 29, 10964-10972.

(76) Incorvati, J. T.; Wan, L. F.; Key, B.; Zhou, D.; Liao, C.; Fuoco, L.; Holland, M.; Wang, H.; Prendergast, D.; Poeppelmeier, K. R.; Vaughey, J. T. Reversible Magnesium Intercalation into a Layered Oxyfluoride Cathode. Chem. Mater. 2016, 28 (1), 17-20.

(77) Fan, X.; Garai, S.; Gaddam, R. R.; Menezes, P. V.; Dubal, D. P.; Yamauchi, Y.; Menezes, P. W.; Nanjundan, A. K.; Zhao, X. S. Uncovering Giant Nanowheels for Magnesium Ion-Based Batteries. Mater. Today Chem. 2020, 16, 100221.

(78) Gautam, G. S.; Sun, X.; Duffort, V.; Nazar, L. F.; Ceder, G. Impact of Intermediate Sites on Bulk Diffusion Barriers: Mg Intercalation in $\mathrm{Mg}_{2} \mathrm{Mo}_{3} \mathrm{O}_{8}$. J. Mater. Chem. A 2016, 4 (45), 17643-17648.

(79) Ishida, N.; Nakamura, Y.; Mandai, T.; Kitamura, N.; Idemoto, Y. Synthesis, Cathode Property and Crystal, Electronic and Local Structures of $\mathrm{Mg}_{2} \mathrm{Mo}_{3} \mathrm{O}_{8}$ as $\mathrm{Mg}$ Rechargeable Battery Cathode Material. Solid State Ionics 2020, 354, 115413. https://doi.org/10.1016/j.ssi.2020.115413.

(80) Ishida, N.; Nishigami, R.; Kitamura, N.; Idemoto, Y. Crystal Structure Analysis and Electrochemical Properties of Chemically Delithiated $\mathrm{Li}_{0.13} \mathrm{Mn}_{0.54} \mathrm{Ni}_{0.13} \mathrm{Co}_{0.13} \mathrm{O}_{21}$ $\not$ as Cathode Material for Rechargeable Mg Batteries. Chem. Lett. 2017, 46 (10), 1508-1511.

(81) Ishida, N.; Yamazaki, N.; Mandai, T.; Kitamura, N.; Idemoto, Y. Crystal Structures and Cathode Properties of Chemically and Electrochemically Delithiated $\mathrm{Li}_{1} \mathrm{Ni}_{0.5} \mathrm{Mn}_{0.5} \mathrm{O}_{2}$ with Applications to $\mathrm{Mg}$ Rechargeable Batteries. J. Electrochem. Soc. 2020,167 (10), 100547.

(82) Asif, M.; Rashad, M.; Shah, J. H.; Zaidi, S. D. A. Surface Modification of Tin Oxide through Reduced Graphene Oxide as Highly Efficient Cathode Material for Magnesium-Ion Batteries. J. Colloid Interface Sci. 2019, 561, 818-828

(83) Makino, K.; Katayama, Y.; Miura, T.; Kishi, T. Magnesium Insertion into $\mathrm{Mg}_{0.5+y}\left(\mathrm{Fe}_{\mathrm{y}} \mathrm{Ti}_{1-y}\right)_{2}\left(\mathrm{PO}_{4}\right)_{3}$. J. Power Sources 2001, 97-98, 512-514.

(84) Makino, K.; Katayama, Y.; Miura, T.; Kishi, T. Electrochemical Insertion of Magnesium to $\mathrm{Mg}_{0.5} \mathrm{Ti}_{2}\left(\mathrm{PO}_{4}\right)_{3}$. J. Power Sources 2001, $99(1-2)$, 66-69.

(85) Makino, K.; Katayama, Y.; Miura, T.; Kishi, T. Preparation and Electrochemical Magnesium Insertion Behaviors of $\mathrm{Mg}_{0.5+y}\left(\mathrm{MeyTi}_{1-\mathrm{y}}\right)_{2}\left(\mathrm{PO}_{4}\right)_{3}$ $(\mathrm{Me}=\mathrm{Cr}$, Fe). J. Power Sources 2002, $112(1), 85-89$.

(86) Sun, X.; Blanc, L.; Nolis, G. M.; Bonnick, P.; Cabana, J.; Nazar, L. F. NaV ${ }_{1.25} \mathrm{Ti}_{0.75} \mathrm{O}_{4}$ : A Potential Post-Spinel Cathode Material for Mg Batteries. Chem. Mater. 2018, 30 (1), 121-128.

(87) Cabello, M.; Ortiz, G. F.; Lavela, P.; Tirado, J. L. On the Beneficial Effect of $\mathrm{MgCl}_{2}$ as Electrolyte Additive to Improve the Electrochemical 
Performance of $\mathrm{Li}_{4} \mathrm{Ti}_{5} \mathrm{O}_{12}$ as Cathode in Mg Batteries. Nanomaterials 2019, 9 (3), 484.

(88) Lee, B.; Jo, E.; Choi, J.; Kim, J. H.; Chang, W.; Yu, S.; Kim, H. S.; Oh, S. H. Cr-Doped Lithium Titanate Nanocrystals as Mg lon Insertion Materials for Mg Batteries. J. Mater. Chem. A 2019, 7 (44), 25619-25627.

(89) Wu, N.; Lyu, Y. C.; Xiao, R. J.; Yu, X.; Yin, Y. X.; Yang, X. Q.; Li, H.; Gu, L.; Guo, Y. G. A Highly Reversible, Low-Strain Mg-lon Insertion Anode Material for Rechargeable Mg-Ion Batteries. NPG Asia Mater. 2014, 6 (8), 1-7.

(90) Wu, N.; Yin, Y. X.; Guo, Y. G. Size-Dependent Electrochemical Magnesium Storage Performance of Spinel Lithium Titanate. Chem. - An Asian J. 2014, 9 (8), 2099-2102.

(91) Gregory, T. D.; Hoffman, R. J.; Winterton, R. C. Nonaqueous Electrochemistry of Magnesium: Applications to Energy Storage. J. Electrochem. Soc. 1990, 137 (3), 775-780.

(92) Wang, R.; Chung, C. C.; Liu, Y.; Jones, J. L.; Augustyn, V. Electrochemical Intercalation of $\mathrm{Mg}^{2+}$ into Anhydrous and Hydrated Crystalline Tungsten Oxides. Langmuir 2017, 33 (37), 9314-9323.

(93) Yaghoobnejad Asl, H.; Manthiram, A. Mass Transfer of Divalent lons in an Oxide Host: Comparison of $\mathrm{Mg}^{2+}$ and $\mathrm{Zn}^{2+}$ Diffusion in Hexagonal $\mathrm{K}_{\mathrm{x}} \mathrm{W}_{3} \mathrm{O}_{9}$ Bronze. Chem. Mater. 2019, 31 (7), 2296-2307.

(94) Pereira-Ramos, J. P.; Messina, R.; Perichon, J. Electrochemical Formation of a Magnesium Vanadium Bronze $\mathrm{Mg}_{\mathrm{x}} \mathrm{V}_{2} \mathrm{O}_{5}$ in Sulfone-Based Electrolytes at $150^{\circ} \mathrm{C}$. J. Electroanal. Chem. 1987, 218 (1-2), 241-249.

(95) Kanatzidis, M. G.; Wu, C.-G.; Liu, Y.-J.; DeGroot, D. C.; Schindler, J. L.; Marcy, H. O.; Kannewurf, C. R. Intercalation Of Layered $\mathrm{V}_{2} \mathrm{O}_{5} \mathrm{Xerogel}$ With Polymers. MRS Proc. 1991, 233 (183).

(96) Shklover, V.; Haibach, T.; Reid, F.; Nesper, R.; Novak, P. Crystal Structure of the Product of $\mathrm{Mg}^{2+}$ Insertion into $\mathrm{V}_{2} \mathrm{O}_{5}$ Single Crystals. J. Solid State Chem. 1996, 323 (123), 317-323.

(97) Novák, P.; Shklover, V.; Nesper, R. Magnesium Insertion in Vanadium Oxides: A Structural Study. Zeitschrift fur Phys. Chemie 1994, 185, 51-68.

(98) Novák, P.; Scheifele, W.; Joho, F.; Haas, O. Electrochemical Insertion of Magnesium into Hydrated Vanadium Bronzes. J. Electrochem. Soc. 1995, 142 (8), 2544.

(99) Le, D. B.; Passerini, S.; Coustier, F.; Guo, J.; Soderstrom, T.; Owens, B. B.; Smyrl, W. H. Intercalation of Polyvalent Cations into $V_{2} \mathrm{O}_{5}$ Aerogels. Chem. Mater. 1998, 10 (3), 682-684.

(100) Amatucci, G. G.; Badway, F.; Singhal, A.; Beaudoin, B.; Skandan, G.; Bowmer, T.; Plitz, I.; Pereira, N.; Chapman, T.; Jaworski, R. Investigation of Yttrium and Polyvalent Ion Intercalation into Nanocrystalline Vanadium Oxide. J. Electrochem. Soc. 2001, 148 (8), A940- 
A950.

(101) Imamura, D.; Miyayama, M.; Hibino, M.; Kudo, T. Mg Intercalation Properties into $\mathrm{V}_{2} \mathrm{O}_{5}$ Gel/Carbon Composites under High-Rate Condition. J. Electrochem. Soc. 2003, 150 (6), A753.

(102) Imamura, D.; Miyayama, M. Characterization of Magnesium-Intercalated $\mathrm{V}_{2} \mathrm{O}_{5} /$ Carbon Composites. Solid State lonics 2003, 161 (1-2), 173-180.

(103) Yu, L.; Zhang, X. Electrochemical Insertion of Magnesium Ions into $\mathrm{V}_{2} \mathrm{O}_{5}$ from Aprotic Electrolytes with Varied Water Content. J. Colloid Interface Sci. 2004, 278 (1), 160-165.

(104) Inamoto, M.; Kurihara, H.; Yajima, T. Electrode Performance of Vanadium Pentoxide Xerogel Prepared by Microwave Irradiation as an Active Cathode Material for Rechargeable Magnesium Batteries. Electrochemistry 2012, 80 (6), 421-422.

(105) Tepavcevic, S.; Liu, Y.; Zhou, D.; Lai, B.; Maser, J.; Zuo, X.; Chan, H.; Král, P.; Johnson, C. S.; Stamenkovic, V.; Markovic, N. M.; Rajh, T. Nanostructured Layered Cathode for Rechargeable Mg-Ion Batteries. ACS Nano 2015, 9 (8), 8194-8205.

(106) Lee, S. H.; DiLeo, R. A.; Marschilok, A. C.; Takeuchi, K. J.; Takeuchi, E. S. Sol Gel Based Synthesis and Electrochemistry of Magnesium Vanadium Oxide: A Promising Cathode Material for Secondary Magnesium lon Batteries. ECS Electrochem. Lett. 2014, 3 (8), A87-A90.

(107) An, Q.; Li, Y.; Deog Yoo, H.; Chen, S.; Ru, Q.; Mai, L.; Yao, Y. Graphene Decorated Vanadium Oxide Nanowire Aerogel for Long-Cycle-Life Magnesium Battery Cathodes. Nano Energy 2015, 18, 265-272.

(108) Arthur, T. S.; Kato, K.; Germain, J.; Guo, J.; Glans, P. A.; Liu, Y. S.; Holmes, D.; Fan, X.; Mizuno, F. Amorphous $\mathrm{V}_{2} \mathrm{O}_{5}-\mathrm{P}_{2} \mathrm{O}_{5}$ as High-Voltage Cathodes for Magnesium Batteries. Chem. Commun. 2015, 51 (86), 15657-15660.

(109) Vujković, M.; Pašti, I.; Simatović, I. S.; Šljukić, B.; Milenković, M.; Mentus, S. The Influence of Intercalated Ions on Cyclic Stability of $\mathrm{V}_{2} \mathrm{O}_{5}$ /Graphite Composite in Aqueous Electrolytic Solutions: Experimental and Theoretical Approach. Electrochim. Acta 2015, 176, 130140.

(110) Du, X.; Huang, G.; Qin, Y.; Wang, L. Solvothermal Synthesis of $\mathrm{GO} / \mathrm{V}_{2} \mathrm{O}_{5}$ Composites as a Cathode Material for Rechargeable Magnesium Batteries. RSC Adv. 2015, 5 (93), 76352-76355.

(111) Jiao, L. F.; Yuan, H. T.; Si, Y. C.; Wang, Y. J.; Wang, Y. M. Synthesis of $\mathrm{Cu}_{0.1}$-Doped Vanadium Oxide Nanotubes and Their Application as Cathode Materials for Rechargeable Magnesium Batteries. Electrochem. commun. 2006, 8 (6), 1041-1044.

(112) Jiao, L.; Yuan, H.; Si, Y.; Wang, Y.; Cao, J.; Gao, X.; Zhao, M.; Zhou, X.; Wang, Y. Electrochemical Insertion of Magnesium in Open-Ended Vanadium Oxide Nanotubes. J. Power Sources 2006, 156 (2), 673-676.

(113) Jiao, L.; Yuan, H.; Wang, Y.; Cao, J.; Wang, Y. Mg Intercalation Properties into Open-Ended Vanadium Oxide Nanotubes. Electrochem. 
commun. 2005, $7(4), 431-436$.

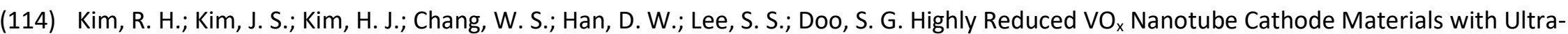
High Capacity for Magnesium Ion Batteries. J. Mater. Chem. A 2014, 2 (48), 20636-20641.

(115) Cheng, Y.; Shao, Y.; Raju, V.; Ji, X.; Mehdi, B. L.; Han, K. S.; Engelhard, M. H.; Li, G.; Browning, N. D.; Mueller, K. T.; Liu, J. Molecular Storage of Mg lons with Vanadium Oxide Nanoclusters. Adv. Funct. Mater. 2016, 26 (20), 3446-3453.

(116) Cabello, M.; Nacimiento, F.; Alcántara, R.; Lavela, P.; Ortiz, G.; Tirado, J. L. Nanobelts of Beta-Sodium Vanadate as Electrode for Magnesium and Dual Magnesium-Sodium Batteries. J. Electrochem. Soc. 2016, 163 (13), A2781-A2790.

(117) Sa, N.; Wang, H.; Proffit, D. L.; Lipson, A. L.; Key, B.; Liu, M.; Feng, Z.; Fister, T. T.; Ren, Y.; Sun, C. J.; Vaughey, J. T.; Fenter, P. A.; Persson, K. A.; Burrell, A. K. Is Alpha- $\mathrm{V}_{2} \mathrm{O}_{5}$ a Cathode Material for Mg Insertion Batteries? J. Power Sources 2016, 323, 44-50.

(118) Kaveevivitchai, W.; Jacobson, A. J. High Capacity Rechargeable Magnesium-Ion Batteries Based on a Microporous Molybdenum-Vanadium Oxide Cathode. Chem. Mater. 2016, 28 (13), 4593-4601.

(119) Yin, J.; Pelliccione, C. J.; Lee, S. H.; Takeuchi, E. S.; Takeuchi, K. J.; Marschilok, A. C. Communication-Sol-Gel Synthesized Magnesium Vanadium Oxide, $\mathrm{Mg}_{\mathrm{x}} \mathrm{V}_{2} \mathrm{O}_{5} \cdot \mathrm{nH}_{2} \mathrm{O}$ : The Role of Structural $\mathrm{Mg}^{2+}$ on Battery Performance . J. Electrochem. Soc. 2016,163 (9), A1941-A1943.

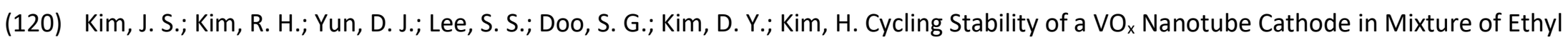
Acetate and Tetramethylsilane-Based Electrolytes for Rechargeable Mg-lon Batteries. ACS Appl. Mater. Interfaces 2016, 8 (40), 2665726663.

(121) Sa, N.; Kinnibrugh, T. L.; Wang, H.; Sai Gautam, G.; Chapman, K. W.; Vaughey, J. T.; Key, B.; Fister, T. T.; Freeland, J. W.; Proffit, D. L.; Chupas, P. J.; Ceder, G.; Bareno, J. G.; Bloom, I. D.; Burrell, A. K. Structural Evolution of Reversible Mg Insertion into a Bilayer Structure of $\mathrm{V}_{2} \mathrm{O}_{5} \cdot \mathrm{nH}_{2} \mathrm{O}$ Xerogel Material. Chem. Mater. 2016, $28(9), 2962-2969$.

(122) Perera, S. D.; Archer, R. B.; Damin, C. A.; Rhodes, C. P.; Mendoza-Cruz, R.; Rhodes, C. P. Controlling Interlayer Interactions in Vanadium Pentoxide-Poly (Ethylene Oxide) Nanocomposites for Enhanced Magnesium-lon Charge Transport and Storage. J. Power Sources 2017, 343, 580-591.

(123) Lim, S. C.; Lee, J.; Kwak, H. H.; Heo, J. W.; Chae, M. S.; Ahn, D.; Jang, Y. H.; Lee, H.; Hong, S. T. Unraveling the Magnesium-lon Intercalation Mechanism in Vanadium Pentoxide in a Wet Organic Electrolyte by Structural Determination. Inorg. Chem. 2017, 56 (14), 7668-7678.

(124) Miao, X.; Chen, Z.; Wang, N.; Nuli, Y.; Wang, J.; Yang, J.; Hirano, S. ichi. Electrospun $\mathrm{V}_{2} \mathrm{MoO}_{8}$ as a Cathode Material for Rechargeable Batteries with Mg Metal Anode. Nano Energy 2017, 34, 26-35.

(125) Mukherjee, A.; Sa, N.; Phillips, P. J.; Burrell, A.; Vaughey, J.; Klie, R. F. Direct Investigation of Mg Intercalation into Orthorhombic $\mathrm{V}_{2} \mathrm{O}_{5}$ 
Cathode Using Atomic Resolution Transmission Electron Microscopy. Chem. Mater. 2017, 29 (5), 2218-2226. https://doi.org/10.1021/acs.chemmater.6b05089.

(126) Tang, H.; Xu, N.; Pei, C.; Xiong, F.; Tan, S.; Luo, W.; An, Q.; Mai, L. $\mathrm{H}_{2} \mathrm{~V}_{3} \mathrm{O}_{8}$ Nanowires as High-Capacity Cathode Materials for MagnesiumBased Battery. ACS Appl. Mater. Interfaces 2017, 9 (34), 28667-28673.

(127) Christensen, C. K.; Bøjesen, E. D.; Sørensen, D. R.; Kristensen, J. H.; Mathiesen, J. K.; Iversen, B. B.; Ravnsbæk, D. B. Structural Evolution during Lithium-and Magnesium-Ion Intercalation in Vanadium Oxide Nanotube Electrodes for Battery Applications. ACS Appl. Nano Mater. 2018, 1 (9), 5071-5082.

(128) Rashad, M.; Zhang, H.; Asif, M.; Feng, K.; Li, X.; Zhang, H. Low-Cost Room-Temperature Synthesis of $\mathrm{NaV}_{3} \mathrm{O}_{8} \cdot 1.69 \mathrm{H}_{2} \mathrm{O} \mathrm{Nanobelts} \mathrm{for} \mathrm{Mg}$ Batteries. ACS Appl. Mater. Interfaces 2018, 10 (5), 4757-4766.

(129) Sun, J. Z. Study of $\mathrm{MgV}_{2} \mathrm{O}_{6}$ as Cathode Material for Secondary Magnesium Batteries. Asian J. Chem. 2011,23 (3), $1399-1400$.

(130) Zhou, L.; Liu, Q.; Zhang, Z.; Zhang, K.; Xiong, F.; Tan, S.; An, Q.; Kang, Y. M.; Zhou, Z.; Mai, L. Interlayer-Spacing-Regulated VOPO 4 Nanosheets with Fast Kinetics for High-Capacity and Durable Rechargeable Magnesium Batteries. Adv. Mater. 2018,30 (32), e1801984.

(131) Aragón, M. J.; Lavela, P.; Recio, P.; Alcántara, R.; Tirado, J. L. On the Influence of Particle Morphology to Provide High Performing Chemically Desodiated C@NaV $2\left(\mathrm{PO}_{4}\right)_{3}$ as Cathode for Rechargeable Magnesium Batteries. J. Electroanal. Chem. 2018, 827 (July), 128-136.

(132) Ji, X.; Chen, J.; Wang, F.; Sun, W.; Ruan, Y.; Miao, L.; Jiang, J.; Wang, C. Water-Activated VOPO 4 for Magnesium lon Batteries. Nano Lett. 2018, $18(10), 6441-6448$.

(133) Attias, R.; Salama, M.; Hirsch, B.; Gofer, Y.; Aurbach, D. Solvent Effects on the Reversible Intercalation of Magnesium-lons into $\mathrm{V}_{2} \mathrm{O}_{5}$ Electrodes. ChemElectroChem 2018, 5 (22), 3514-3524.

(134) Andrews, J. L.; Mukherjee, A.; Yoo, H. D.; Parija, A.; Marley, P. M.; Fakra, S.; Prendergast, D.; Cabana, J.; Klie, R. F.; Banerjee, S. Reversible $\mathrm{Mg}$-Ion Insertion in a Metastable One-Dimensional Polymorph of $\mathrm{V}_{2} \mathrm{O}_{5}$. Chem 2018, 4 (3), 564-585.

(135) Luo, T.; Liu, Y.; Su, H.; Xiao, R.; Huang, L.; Xiang, Q.; Zhou, Y.; Chen, C. Nanostructured-VO 2 (B): A High-Capacity Magnesium-lon Cathode and Its Electrochemical Reaction Mechanism. Electrochim. Acta 2018, 260, 805-813.

(136) Drosos, C.; Jia, C.; Mathew, S.; Palgrave, R. G.; Moss, B.; Kafizas, A.; Vernardou, D. Aerosol-Assisted Chemical Vapor Deposition of $V_{2} \mathrm{O}_{5}$ cathodes with High Rate Capabilities for Magnesium-Ion Batteries. J. Power Sources 2018, 384, 355-359.

(137) Verrelli, R.; Black, A. P.; Pattanathummasid, C.; Tchitchekova, D. S.; Ponrouch, A.; Oró-Solé, J.; Frontera, C.; Bardé, F.; Rozier, P.; Palacín, M. R. On the Strange Case of Divalent lons Intercalation in $\mathrm{V}_{2} \mathrm{O}_{5}$. J. Power Sources 2018, 407, 162-172.

(138) Yoo, H. D.; Jokisaari, J. R.; Yu, Y.-S. S.; Kwon, B. J.; Hu, L.; Kim, S.; Han, S.-D. D.; Lopez, M.; Lapidus, S. H.; Nolis, G. M.; Ingram, B. J.; Bolotin, 
I. L.; Ahmed, S.; Klie, R. F.; Vaughey, J. T.; Fister, T. T.; Cabana, J. Intercalation of Magnesium into a Layered Vanadium Oxide with High Capacity. ACS Energy Lett. 2019, 4, 1528-1534.

(139) Wu, D.; Zeng, J.; Hua, H.; Wu, J.; Yang, Y.; Zhao, J. $\mathrm{NaV}_{6} \mathrm{O}_{15}$ : A Promising Cathode Material for Insertion / Extraction of $\mathrm{Mg}^{2+}$ with Excellent Cycling Performance. Nano Res. 2019, 2 (1), 10-12.

(140) Tang, H.; Xiong, F.; Jiang, Y.; Pei, C.; Tan, S.; Yang, W.; Li, M.; An, Q.; Mai, L. Alkali lons Pre-Intercalated Layered Vanadium Oxide Nanowires for Stable Magnesium lons Storage. Nano Energy 2019, 58, 347-354.

(141) Attias, R.; Salama, M.; Hirsch, B.; Pant, R.; Gofer, Y.; Aurbach, D. Anion Effects on Cathode Electrochemical Activity in Rechargeable Magnesium Batteries: A Case Study of $\mathrm{V}_{2} \mathrm{O}_{5}$. ACS Energy Lett. 2019, 4 (1), 209-214.

(142) Xu, Y.; Deng, X.; Li, Q.; Zhang, G.; Xiong, F.; Tan, S.; Wei, Q.; Lu, J.; Li, J.; An, Q.; Mai, L. Vanadium Oxide Pillared by Interlayer Mg ${ }^{2+}$ Ions and Water as Ultralong-Life Cathodes for Magnesium-Ion Batteries. Chem 2019, 5 (5), 1194-1209.

(143) Kim, D.; Ryu, J. H. Amorphous $\mathrm{V}_{2} \mathrm{O}_{5}$ Positive Electrode Materials by Precipitation Method in Magnesium Rechargeable Batteries. Electron. Mater. Lett. 2019, 15 (4), 415-420.

(144) Deng, X.; Xu, Y.; An, Q.; Xiong, F.; Tan, S.; Wu, L.; Mai, L. Manganese Ion Pre-Intercalated Hydrated Vanadium Oxide as a HighPerformance Cathode for Magnesium Ion Batteries. J. Mater. Chem. A 2019, 7 (17), 10644-10650.

(145) Medina, A.; Cabello, M.; Alcántara, R.; Pérez-Vicente, C.; Tirado, J. L. Theoretical and Experimental Study on the Electrochemical Behavior of Beta-Sodium Vanadate in Rechargeable Magnesium Batteries Using Several Electrolyte Solutions. J. Electrochem. Soc. 2020, 167 (7), 070512.

(146) Johnson, I. D.; Nolis, G.; McColl, K.; Wu, Y. A.; Thornton, D.; Hu, L.; Yoo, H. D.; Freeland, J. W.; Corà, F.; Cockcroft, J. K.; Parkin, I. P.; Klie, R. F.; Cabana, J.; Darr, J. A. Probing Mg Intercalation in the Tetragonal Tungsten Bronze Framework $\mathrm{V}_{4} \mathrm{Nb}_{18} \mathrm{O}_{55}$. Inorg. Chem. 2020, 59 (14), 9783-9797.

(147) Idemoto, Y.; Kawakami, N.; Ishida, N.; Kitamura, N. Synthesis, Electrochemical Properties and Changes of Crystal and Electronic Structures in Charge/Discharge Process of Spinel Type Cathode-Materials $\mathrm{Mg}\left(\mathrm{Mg}_{0.5} \mathrm{~V}_{1.5-\mathrm{x}} \mathrm{Ni}_{\mathrm{x}}\right) \mathrm{O} 4(\mathrm{x}=0,0.1,0.2,0.3)$ for Magnesium Secondary Batteries. Electrochemistry 2019, 87 (5), 281-288.

(148) Idemoto, Y.; Kawakami, N.; Ishida, N.; Kitamura, N. Synthesis, Electrochemical Properties, and Changes in Crystal and Electronic Structures during Charge/Discharge Process of Spinel-Type Cathode Materials $\mathrm{Mg}_{4} \mathrm{~V}_{5-\mathrm{x}} \mathrm{Ni}_{\mathrm{x}} \mathrm{O}_{12}(\mathrm{x}=0,0.3,0.6,1.0)$ for Magnesium Secondary Batteries. J. Power Sources 2020, 455, 227962.

(149) Fu, Q.; Sarapulova, A.; Trouillet, V.; Zhu, L.; Fauth, F.; Mangold, S.; Welter, E.; Indris, S.; Knapp, M.; Dsoke, S.; Bramnik, N.; Ehrenberg, H. In Operando Synchrotron Diffraction and in Operando X-Ray Absorption Spectroscopy Investigations of Orthorhombic $\mathrm{V}_{2} \mathrm{O}_{5} \mathrm{Nanowires}$ as 
Cathode Materials for Mg-Ion Batteries. J. Am. Chem. Soc. 2019, 141 (6), 2305-2315.

(150) Henry, H. K.; Johnston, B.; Liau, D.; Sahadeo, E.; Lee, S. B. Dual Effect of Structure and Hydration on Magnesium-Ion Insertion into Electrodeposited V2O5 Thin Films. J. Electrochem. Soc. 2020, 167, 110523

(151) Mukherjee, A.; Taragin, S.; Aviv, H.; Perelshtein, I.; Noked, M. Rationally Designed Vanadium Pentoxide as High Capacity Insertion Material for Mg-Ion. Adv. Funct. Mater. 2020, 2003518, 1-9.

(152) Hu, L.; Jokisaari, J. R.; Kwon, B. J.; Yin, L.; Kim, S.; Park, H.; Lapidus, S. H.; Klie, R. F.; Key, B.; Zapol, P.; Ingram, B. J.; Vaughey, J. T.; Cabana, J. High Capacity for $\mathrm{Mg}^{2+}$ Deintercalation in Spinel Vanadium Oxide Nanocrystals. ACS Energy Lett. 2020, 5 (8), $2721-2727$.

(153) Zuo, C.; Tang, W.; Lan, B.; Xiong, F.; Tang, H.; Dong, S.; Zhang, W.; Tang, C.; Li, J.; Ruan, Y.; Xi, S.; An, Q.; Luo, P. Unexpected Discovery of Magnesium-Vanadium Spinel Oxide Containing Extractable $\mathrm{Mg}^{2+}$ as a High-Capacity Cathode Material for Magnesium Ion Batteries. Chem. Eng. J. 2021, 405, 127005..

(154) Zhu, Y.; Huang, G.; Yin, J.; Lei, Y.; Emwas, A.-H.; Yu, X.; Mohammed, O. F.; Alshareef, H. N. Hydrated $\mathrm{Mg}_{\times} \mathrm{V}_{5} \mathrm{O}_{12} \mathrm{Cathode}$ with Improved $\mathrm{Mg}^{2+}$ Storage Performance. 2020, 10 (45), 2002128.

(155) Pei, C.; Jin, M.; Yin, Y.; Xiong, F.; Jiang, Y.; Yuan, X.; Wang, F.; An, Q. Intercalation-Type $\mathrm{V}_{2} \mathrm{O}_{3}$ with Fast $\mathrm{Mg}^{2+}$ Diffusion Kinetics for HighCapacity and Long-Life Mg-Ion Storage. ACS Sustain. Chem. Eng. 2020, 8 (43), 16164-16171.

(156) Lopez, M.; Yoo, H. D.; Hu, L.; Andrews, J. L.; Banerjee, S.; Cabana, J. Does Water Enhance Mg Intercalation in Oxides? The Case of a Tunnel Framework. ACS Energy Lett. 2020, 5 (11), 3357-3361.

(157) Johnson, I. D.; Nolis, G.; Yin, L.; Yoo, H. D.; Parajuli, P.; Mukherjee, A.; Andrews, J. L.; Lopez, M.; Klie, R. F.; Banerjee, S.; Ingram, B. J.; Lapidus, S.; Cabana, J.; Darr, J. A. Enhanced Charge Storage of Nanometric $\zeta-\mathrm{V}_{2} \mathrm{O}_{5}$ in Mg Electrolytes. Nanoscale 2020, 12, 22150-22160. 